\title{
Hard X-Ray Waveguide Optics
}

\author{
DisSERTATION \\ zur Erlangung des Doktorgrades \\ der Mathematisch-Nuturwissenschaftlichen Fakultäten \\ der Georg-August-Universität zu Göttingen
}

\author{
vorgelegt von \\ ANSGAR JARRE \\ aus Rheinfelden (Baden)
}

Göttingen, im Juni 2005 
D7

Referent: Prof. Dr Tim Salditt

Koreferent Prof. Dr. Lauterborn

Tag der mündlichen Prüfung: 19.07.2005 

- 4 - 


\section{Contents}

$\begin{array}{lll}1 & \text { Introduction } & 7\end{array}$

2 Hard x-ray micro beam production $\quad 9$

2.1 Introduction . . . . . . . . . . . . . . . . 9

2.2 Fresnel lenses . . . . . . . . . . . . . . . . . . . . . . . . . 9 9

2.3 Bragg Fresnel lenses . . . . . . . . . . . . . . . . . . . 11

2.4 Compound refractive lenses . . . . . . . . . . . . . . . . 13

2.5 Capillary optics . . . . . . . . . . . . . . . . . . . . . . . 14

2.6 Kirkpatrick-Baez Mirrors . . . . . . . . . . . . . . . . . . . . . . . 14

2.7 Comparison of microfocusing optics . . . . . . . . . . . . 15

$\begin{array}{lll}3 & \text { Theory of dielectric x-ray waveguides } & 17\end{array}$

3.1 Planar waveguides . . . . . . . . . . . . . . . 17

3.1.1 Transverse electric modes . . . . . . . . . . . . . . . . 19

3.1.2 Limits of x-ray nano-beam production . . . . . . . . . . 23

3.2 Two dimensionally confining waveguides . . . . . . . . . . . . . 26

3.3 Coupling schemes for hard x-rays . . . . . . . . . . . . . . 30

3.3.1 Resonant Beam Coupling - RBC . . . . . . . . . . 30

3.3.2 Front Coupling . . . . . . . . . . . . . . . 36

3.4 The farfield distribution . . . . . . . . . . . . . . . . . . . . 38

3.4.1 Farfield of a waveguide grating . . . . . . . . . . . . . 42

4 Fabrication of X-ray nanostructures 43

4.1 Thin films . . . . . . . . . . . . . . . . . 44

4.1.1 Spincoating .................... 44

4.1.2 Electron beam evaporation . . . . . . . . . . . . . . . 46

4.1.3 Magnetron sputtering . . . . . . . . . . . . . . . . . 47

4.2 2DWG-fabrication using Focused ion beam . . . . . . . . . . . . . . 49 
4.3 Templating from spider silk fibers . . . . . . . . . . . . . . . 51

4.4 Electron beam lithography . . . . . . . . . . . . . . . . . 55

4.4.1 V-and U-grooves . . . . . . . . . . . . . . . 57

4.4 .2 Polymer core waveguides . . . . . . . . . . . . . 59

5 Experimental I: White beam RBCs 63

5.1 Introduction . . . . . . . . . . . . . . . . . . . 63

5.2 Experimental setup . . . . . . . . . . . . . . 64

5.3 Experiments . . . . . . . . . . . . . . . . 66

6 Experimental II: 2D-Waveguides $\quad 73$

6.1 Introduction . . . . . . . . . . . . . . . . . . . . 73

6.2 FIB-fabricated waveguides and silk templates . . . . . . . . . 74

6.3 Lithographically fabricated waveguides . . . . . . . . . . . . . 79

6.3.1 V-shaped guides held in a bench vice . . . . . . . . . . . 79

6.3.2 Experiments with a bonded grating . . . . . . . . . . 81

6.3.3 Polymer core waveguides . . . . . . . . . . . . . 83

$\begin{array}{llr}7 & \text { Summary } & 89\end{array}$

$\begin{array}{lr}\text { Bibliography } & 92\end{array}$

Acknowledgements . . . . . . . . . . . . . . . . . . . . . . 99

List of Publications . . . . . . . . . . . . . . . . . . . . . 101

Curriculum Vitae . . . . . . . . . . . . . . . . . . 103 


\section{Chapter 1}

\section{Introduction}

According to the Abbe criterion, the maximum resolution in imaging with electromagnetic waves is limited by the wavelength $\lambda$ of the radiation used. Due to their small wavelength, hard x-rays $(\lambda<3 \AA)$ are therefore particularly well suited for the investigation of structure sizes down to the $\AA$ region. X-ray diffraction for example is one of the techniques which benefit from the small wavelength. The cross section $d$ of the x-ray beam used in such experiments however is typically orders of magnitude larger $(d \geq 5 \mu \mathrm{m})$ than the wavelength of the radiation that is used. Therefore structural information is averaged over the size of the macroscopic sample. Focussing x-rays to nm size would overcome these limitations. However, as was already noted by Roentgen (Röntgen. 1895, 1896), reflection and refraction effects of x-ray beams were very small, if at all present. Today we know that the refractive index of matter for hard x-rays is typically only less than $10^{-5}$ away from unity and therfore focusing is difficult. Nevertheless, different reflective, refractive and focussing diffractive optics have been developed. The state of the art of these optics which are described and compared to each other in chapter 2 have been probed in order to achieve smallest beam sizes with sufficient flux.

The present thesis is devoted to the preparation of intense hard x-ray nanobeams using x-ray waveguides. 1974 Spiller and Segmüller have demonstrated that the priciples of guiding electromagnetic radiation can be extended to the x-ray region. However, only since recent years has the quality of synchrotron sources and experimental improvements made it possible to use x-ray waveguides as x-ray optical elements. The theoretical description of the interaction of x-ray waveguides with electromagnetic radiation is subject of chapter 3 followed by a description of those techniques which were used in the present thesis for waveguide fabrication (chap- 
ter 4$)$.

Two different approaches have been investigated in this work to couple the x-ray beam into the guide. In the resonant beam coupling (RBC) scheme, the x-ray beam is coupled via a thin top cladding into the one dimensionally confining guide. X-ray waveguides with enhanced throughput were studied in white synchrotron radiation and the results are compared to simulated data (chapter 5). Two dimensionally confining hard x-ray waveguides have been investigated in a novel front coupling geometry. Here the incoming radiation is coupled directly into the front side of the waveguide making use of an efficient prefocussing by curved mirrors. The results obtained with these devices are reviewed in chapter 6 . 


\section{Chapter 2}

\section{Hard x-ray micro beam production}

\section{$2.1 \quad$ Introduction}

In this chapter we give an overview of the presently investigated reflective, refractive and diffractive optics for the production of x-ray nanobeams. Note, that not only the spot size and the flux density enhancement (gain) are important parameters. All of these techniques go along with individual advantages and disadvantages: While e.g. Compound Refractive Lenses are fairly easy to align, bent mirrors provide the highest flux density. Fresnel lenses are effective and well known from soft $x$-ray microscopes but their fabrication becomes more and more difficult with increasing photon energy. Thus all these techniques are regarded as parts of a rapidly evolving field. A combination of two or more of these techniques can eliminate the deficiencies of a single optic and increase its power for novel applications. A fundamental lower limit for the spot size is predicted by Bergemann et al. (2003) in the range of $\sim 8$ to $20 \mathrm{~nm}$, depending only on the material used for focussing, while Schroer and Lengeler. (2005) claim that $2 \mathrm{~nm}$ spot sizes can be achieved with certain refractive lenses.

\section{$2.2 \quad$ Fresnel lenses}

Fresnel lenses for hard x-rays $(E>5 k e V)$ consist of phase shifting concentric circular rings (zones) positioned on a transparent substrate (see Fig.2.1). They are most commonly produced either by holography or lithography. An incoming plane 
wave (wavelength $\lambda$ ) will either pass a region with zero phase shift (indicated by the white rings in the schematic), or an opaque zone (black rings) ${ }^{1}$. The trick is,
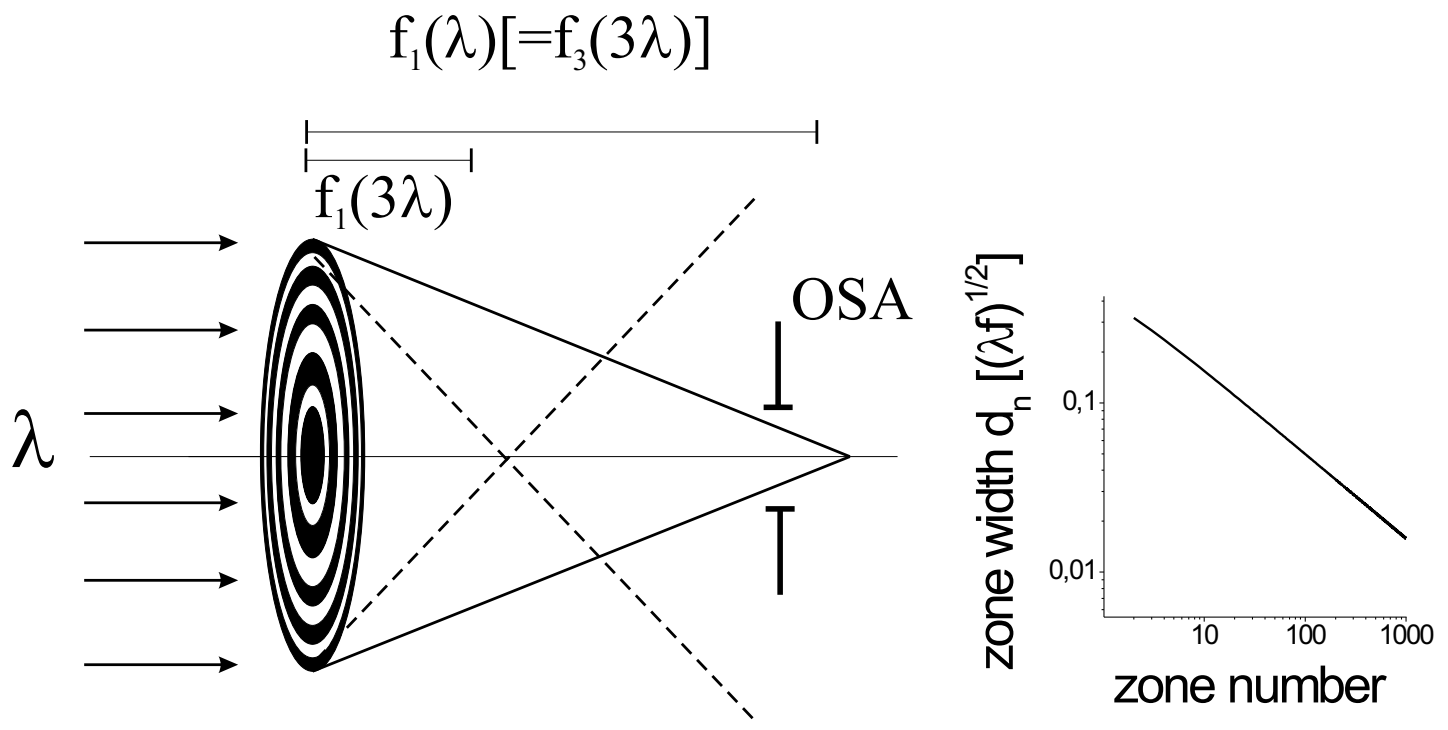

Figure 2.1: left: Principle of a Fresnel Phase Zone Plate for hard x-rays. Longer focal lengths are produced for shorter wavelengths. An order sorting aperture (OSA) can therefore be used to select the photon energy and to reduce the background. right: The zone width of the $n^{\text {th }}$ zone as a function of $n$ in units of $\sqrt{\lambda f}($ At 12.4keV $(\lambda=1 \AA)$ and $f=10 \mathrm{~cm}, \sqrt{\lambda f}=1 \mu \mathrm{m})$.

that all waves passing any of these zones interfere constructively in the focal spot of the lens. With the Pythagorean theorem this is:

$$
f^{2}+r_{n}^{2}=\left(f+\frac{n \lambda}{2}\right)^{2}
$$

with the focal length $f$ of the lens, the radius $r_{n}$ of the $n^{\text {th }}$ zone, the wavelength $\lambda$ and the hypothenuse $f+n \lambda / 2$. This implies, that the zone width is decreasing from the middle of the lens to its outermost zone. For $f \gg n \lambda / 2$ the radius $r_{n}$ of the $\mathrm{n}^{\text {th }}$ zone is then given by:

$$
r_{n}=\sqrt{\lambda f n}
$$

\footnotetext{
${ }^{1}$ In order to increase the efficiency of FZPs, the opaque zones can be replaced by zones with a phase shift of $\lambda / 2$
} 
Thus, the width $d_{n}$ of the $\mathrm{n}^{\text {th }}$ zone is given by

$$
d_{n}=r_{n+1}-r_{n}=\sqrt{\lambda f(n+1)}-\sqrt{\lambda f n} \simeq \sqrt{\frac{\lambda f}{4 n}} \text { (see Fig. 2.1) }
$$

A zone plate with an outermost zone width $a$ therefore consists of $N=\lambda f / 4 a^{2}$ zones and has a radius $R_{n}=\lambda f / 2 a$. The maximum number of zones is limited by the outermost zone width, which in turn is limited by the fabrication process. For soft x-rays $\sim 20 \mathrm{~nm}$ are achieved (Peuker. 2001), for hard x-rays, where higher aspect ratios (height of the zone over its width) are needed to achieve the absorption/phase shift, an outermost zone width of $\sim 100 \mathrm{~nm}$ has been reported (David et al. 2004). The efficiency (i.e. the ratio of intensity in the focal spot over the intensity impinging on the zone plate) of a zone plate of alternately opaque and transmissive zones is given by (Attwood 2000)

$$
\begin{array}{cl}
1 / 4 & m=0 \quad \text { (primary beam) } \\
\eta_{m}=1 / m^{2} \pi^{2} & m \text { odd } \\
0 & m \text { even. }
\end{array}
$$

The efficiency of FZPs can be increased by a factor of four by replacing the opaque zones with phase shifting zones that achieve a $\lambda / 2$ phase shift.

The type of lens described above is called binary phase zone plate, since there are only two states of phase shifting $(0$ and $\lambda / 2)$ or absorption. More elaborate techniques of preparing zone plates are investigated to produce quaternary zone plates, following the same principle, but providing four different phase shift regions $(0, \lambda / 4, \lambda / 2$ and $3 \lambda / 4)$, which further increases the efficiency of the lens and reduces the background (Fabrizio et al. 1999). Technically however, this also limits the outermost zone width towards higher values. Phase zone plates are designed to optimize very different parameters, as there is their efficiency, or their outermost zone width $a$ which determines the spatial resolution $\delta_{r}$ in x-ray microscopy given by $\delta_{r}=1.22 a$, for the first diffraction order as derived from the Rayleigh criterion (Yun et al. 1999).

\subsection{Bragg Fresnel lenses}

Fresnel phase zone plates are not only phase objects, since there is no material which only shifts the phase of the incoming wave. There is also absorption in 
these zones, which not only causes a loss in the maximum achievable efficiency of these devices, but also increases the background. An approach to overcome these limitations is the use of so called Bragg-Fresnel lenses (BFL) which work at a Bragg reflection rather than in transmission. A schematic of such a lens, focusing in one dimension, is shown in Fig 2.2. The thickness $\mathrm{h}$ is chosen such that the

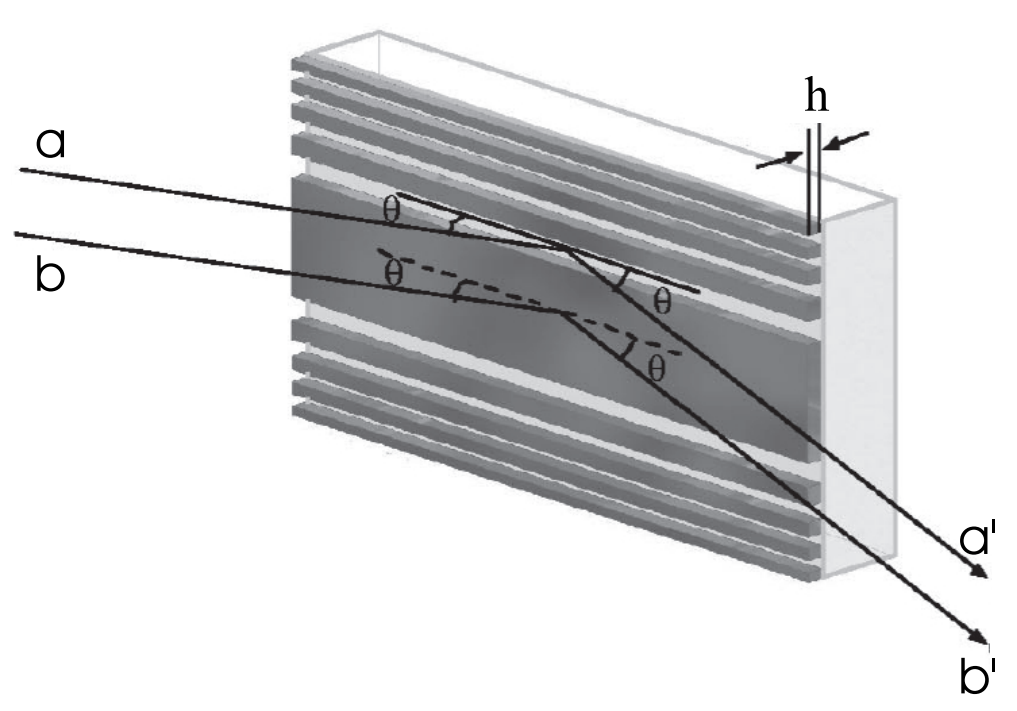

Figure 2.2: Schematic of a linear Bragg-Fresnel lens, adapted from Li et al. (2003)

radiation reflected by the lower surface (a) gains a phase shift $\pi$, as compared to that reflected by the upper surface (b). The width of the zones follow the same law as for FZPs, and consecutively all arguments and calculations are the same for BFLs and FZPs. Two dimensional focusing is achieved by using two BFLs in rectangular geometry. In backscattering experiments (for soft x-rays), circular BFLs can be used which directly produce a two dimensional focusing (Snigirev. 1994). A single device producing a point focus for hard x-rays can be generated by combining BFLs with other focusing techniques, e.g. an elliptical BFL on a bent mirror such as the Kirkpatrick-Baez mirrors (Yasa et al. 2004) which are described below. 


\subsection{Compound refractive lenses}

Since the x-ray index of refraction differs only slightly from unity, lenses - as they are used for optical light - can not be created with reasonable focal lengths. Nevertheless, the 'classical' idea can be used if many of these lenses are placed one behind the other. This is the basic idea of compound refractive lenses (CRLs), see Fig.2.3. Although there is no reason why these lenses could not focus the incoming beam in both, vertical and horizontal direction, only recently two dimensionally focusing biconcave CRLs have been fabricated (Lengeler et al. 2005). Most presently used CRLs focus the x-ray beam in one dimension only. Nevertheless, by aligning two of these devices in crossed geometry on a common optical axis, two dimensional beam compression is achieved. A CRL's focal length $f$ is given by $f=R / 2 N \delta$

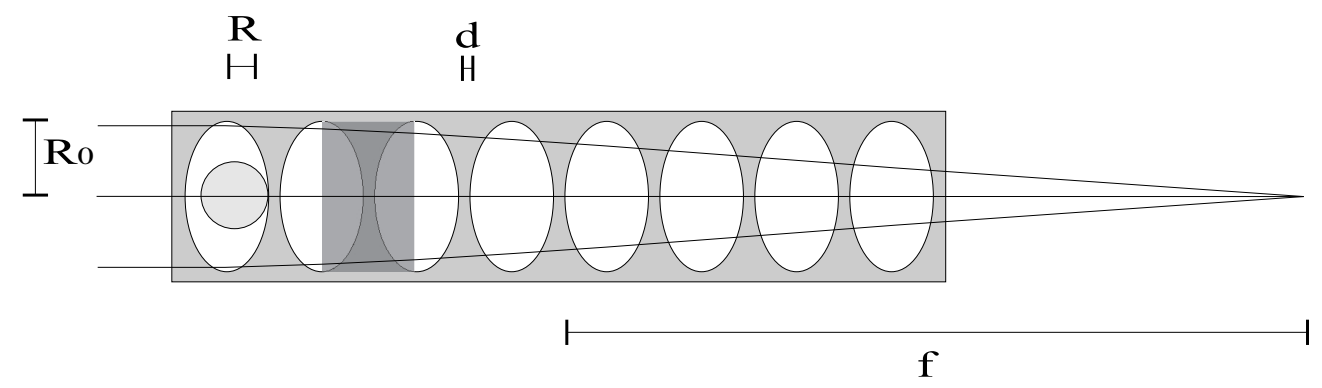

Figure 2.3: Sketch of a parabolic compound refractive lens. One individual lens is shaded dark

(Snigirev et al. 1996), where $\mathrm{N}$ is the number of individual lenses stacked behind each other and $\mathrm{R}$ is the radius of curvature at the apex of the parabola. Note, that since the real part of the refractive index is smaller than one, focussing is achieved by concave lenses. Their experimentally obtained gain ranges from 29 to 177 in publications (Lengeler et al. 1999; Schroer et al. 2003) but design parameters are suggested that might increase the gain to up to $8.4 \times 10^{4}$. Smallest spot sizes are so far achieved in the range of $110 \times 330 \mathrm{~nm}^{2}$ (Schroer et al. 2004) ${ }^{2}$. However, it is currently under discussion if (at least theoretically) spot sizes below the limit predicted by Bergemann et al. (2003) can be achieved with adiabatically focusing lenses where $R$ and $R_{0}$ of each individual lens are adapted to the actual beamsize at the position of the individual lens (Schroer and Lengeler. 2005).

\footnotetext{
${ }^{2} \mathrm{~A}$ spotsize of $47 \times 55 \mathrm{~nm}^{2}$ has been reported in a letter published after this theses had been accomplished (Schroer et al. 2005).
} 


\subsection{Capillary optics}

Capillary optics squeeze an incident synchrotron radiation beam to smallest beam sizes down to 50nm (Bilderback and Thiel 1995). The principle idea is the reduction of the beamsize due to total reflection inside a tapered capillary which is drawn from glass capillaries at high temperature. Since these devices act similar as x-ray waveguides they are not described in detail here. For theoretical analysis of these devices, the reader is referred to the waveguide section of this thesis. Note however, that in most cases capillary optics are employed in incoherent focusing, whereas coherence effects and coherence filtering is important for x-ray waveguides.

\subsection{Kirkpatrick-Baez Mirrors}

The basic idea of the Kirkpatrick-Baez (KB) focussing optic is to demagnify the beam dimensions using the total external reflections of the beam impinging on two elliptically curved mirrors (see Fig. 2.4). It was first proposed for x-rays by Paul Kirkpatrick and Albert V. Baez in 1948 (Kirkpatrick and Baez. 1948). Approximating the elliptical shape of the mirror, its height profile $z(x)$ needed to obtain a minimum spot size can be written as (Susini 1995)

$$
\begin{gathered}
z(x)=\alpha x^{2}\left(1+\beta x+\gamma x^{2}\right) \text { with } \\
\alpha=\frac{\sin \theta}{4 p}\left(1+\frac{p}{q}\right), \beta=\frac{\cos \theta}{2 p}\left(\frac{p}{q}-1\right) \text { and } \gamma=\frac{1}{4 p q}+\frac{5 \cos ^{2} \theta}{16 p^{2}}\left(1-\frac{p}{q}\right)^{2},
\end{gathered}
$$

where $\mathrm{p}$ is the source-mirror center distance, $\mathrm{q}$ is the focal length and $\theta$ is the incidence angle. Such mirror profiles are achieved either by bending of a flat mirror (Hignette et al. 2003), by combination of plasma chemical vaporization and elastic emission machining (i.e. grinding) (Yamauchi et al. 2003) or by differential deposition on a cylindrical substrate which modifies the cylinder to an ellipse (Ice et al. 2000). The incidence angle of the incoming x-ray beam is well below one degree in order to be in total reflection condition, i.e to obtain an achromatic focusing device. Furthermore, the mirror reflectivity is very high providing an efficiency of up to $60 \%$ for two mirrors in rectangular geometry (Dabin et al. 2002). In order to gather a large amount of the primary beam these mirrors must be very long. Typical lengths are in the range of 10 to $20 \mathrm{~cm}$. The mirrors used in the most recent publications consist of pure silicon without coating or Si-substrates with a 


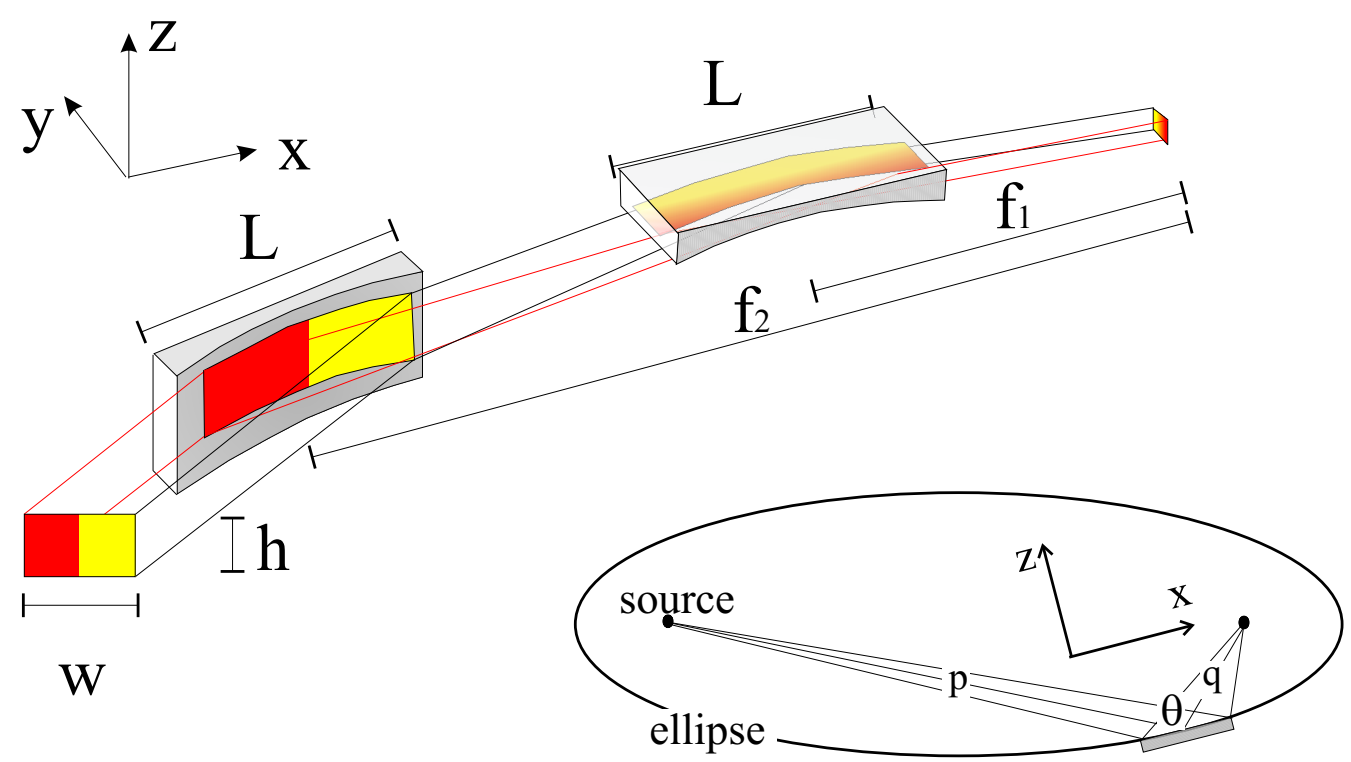

Figure 2.4: Schematic of a Kirkpatrick-Baez focusing setup: Two elliptically curved mirrors with different focal lengths demagnify the beam dimensions in both, horizontal and vertical direction subsequently.

single coating layer or Si-substrates with multilayer coatings, depending on both the preparation technique and the experimental requirements. Spot sizes are reported down to $160 \times 210 \mathrm{~nm}^{2}$ for bent mirrors going along with an estimated gain in flux density of $\sim 3 \cdot 10^{5}$ (Hignette et al. 2003). Ground mirrors were reported to provide a line focus of $120 \mathrm{~nm}$ (Yamura et al. 2003).

\subsection{Comparison of microfocusing optics}

As mentioned above, all hard ( $E \geq 5 \mathrm{keV})$ x-ray micro focusing optics exhibit individual advantages when compared to other techniques. One important parameter, the signal to noise ratio $(\mathrm{s} / \mathrm{n})$ can not be evaluated since in many publications this point is not discussed. Moreover, in a vast majority of the mentioned publications only linear scale profiles of the focal spot are presented.

All of the techniques mentioned here require some time for alignment which can be of importance for experimentalists in particular at synchrotron beamlines where time very often is delimited for experiments. Therefore, 'easy to align'-devices like 


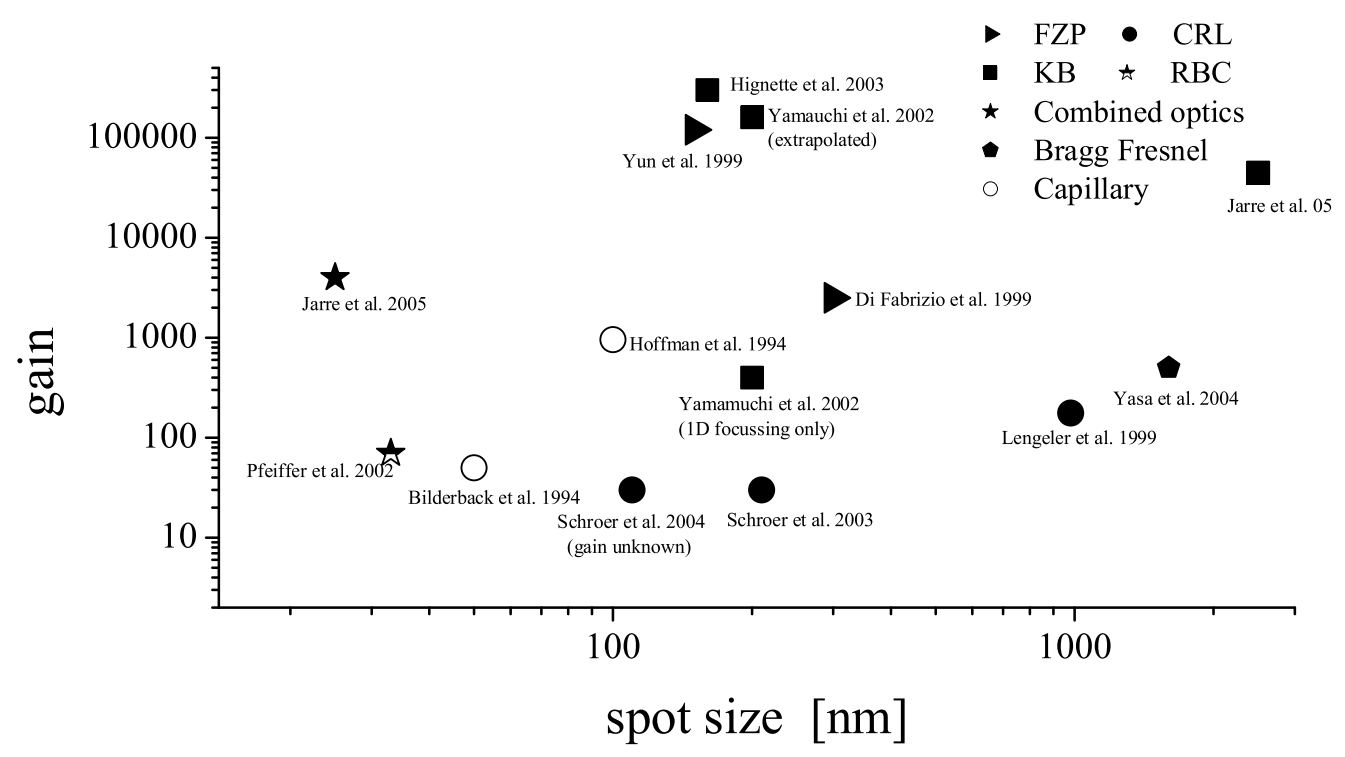

Figure 2.5: Comparison of various focusing techniques for hard $x$-rays: Fresnel Zone Plates (FZP), Kirkpatrick-Baez mirrors (KB), Compound Refractive Lenses (CRL), Bragg-Fresnel-Lenses, Capillary optics, Resonant Beam Couplers (RBC, see chapter 3) and combined KB-and Front-coupling waveguide optics

CRL's have an intrinsic advantage over other techniques. For a lot of applications however, the minimum spot size combined with high flux is of major interest. In Fig. 2.5, a comparison of the techniques is presented with regard to these two parameters. The horizontal axis gives the size of the focused beam in logarithmic scales. Since two dimensional focusing does not mean that the beam offers the same size in horizontal and vertical direction, the smaller dimension is used whenever there is a difference. The vertical shows the flux density enhancement (gain), also in logarithmic scales. Ideal optics would provide the lowest spot size with maximum gain, i.e. it would be found in the top left corner of this graph. 


\section{Chapter 3}

\section{Theory of dielectric x-ray waveguides}

In this chapter we give a mathematical description of hard x-ray waveguiding. We first take a look at the waveguide device and its physical properties. We then start from Maxwell's equations to describe the electric field in the guide and discuss the effect of mode propagation for two different schemes of coupling the electro-magnetic wave into the guide: the resonant beam coupling $(R B C)$ scheme, where the beam is coupled in via a thin cladding layer, and the front coupling scheme, where the beam is coupled directly into the front side of the waveguide.

\subsection{Planar waveguides}

The easiest geometry of a waveguide one can think of is a simple layered system. As the beam is confined in one dimension only, it is called a one dimensional waveguide (1DWG) and gives an excellent insight into the fundamental physical properties. A 1 DWG can be made of just two different materials with their respective index of refraction $n_{j}(j \in[1,2])$. For x-rays $n$ is usually written in the form $n=1-\delta+i \beta$, where

$$
\delta=\lambda^{2} \frac{r_{e} \rho}{2 \pi}
$$

is known as the refractive index decrement, and

$$
\beta=\lambda \frac{\mu}{4 \pi}
$$

as the absorbtion index. $\lambda$ is the x-ray wavelength. It is connected to the x-ray energy $E$ via the relation $\lambda=h c / E$ with Planck's constant $h=6.634 \cdot 10^{-34}$ 


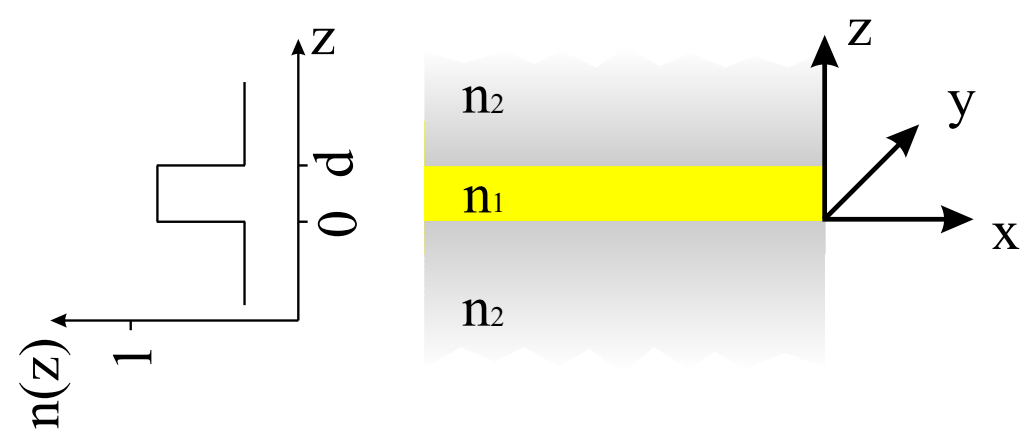

Figure 3.1: The symmetric one-dimensional $x$-ray waveguide consists of only two materials. A thin (thickness d) layer of the material with smaller refractive decrement $\delta_{1}$ and thus higher refractive index $n_{1}$ is sandwiched in-between two thick layers of the second material (refractive index $n_{2}$ ), providing a symmetric profile of the real part of the refractive index $n(z)=1-\delta(z)$.

$\mathrm{Js}^{-1}$ and $c=2.998 \cdot 10^{8} \mathrm{~ms}^{-1}$ the speed of light ${ }^{1} \cdot r_{e}=2.818 \cdot 10^{-15} \mathrm{~m}$ is the classical electron radius, $\rho$ is the electron density of the material, and $\mu$ is its linear absorption coefficient. For hard x-rays (photon energy $E \geq 5 \mathrm{keV}$ ), $\delta$ and $\beta$ are small compared to one, typically in the range of $10^{-5}$ to $10^{-8}$.

For a $1 \mathrm{DWG}$, one thin layer of the material with the smaller $\delta(j=1)$ is sandwiched in-between two layers of the material with a higher $\delta(j=2)$. In a first approach, we will assume, that these so called cladding layers are semi infinite. A sketch of such a structure is shown in Fig. 3.1. The center layer of this device is called the guiding layer. It supports the propagation of electro magnetic waves with a certain field distribution, called modes. A derivation of this effect starts from Maxwell's equations, which in the absence of currents can be written as:

$$
\nabla \times \mathbf{H}=\varepsilon_{0} n^{2} \partial \mathbf{E} / \partial t
$$

and

$$
\nabla \times \mathbf{E}=-\mu_{0} \partial \mathbf{H} / \partial t
$$

where $\nabla$ is the nabla operator $\nabla=\mathbf{e}_{x} \partial / \partial x+\mathbf{e}_{y} \partial / \partial y+\mathbf{e}_{z} \partial / \partial z, \mathbf{E}$ and $\mathbf{H}$ are the electric and magnetic field vectors, $\varepsilon_{o}=8.854 \cdot 10^{-12} \mathrm{As} / \mathrm{Vm}$ is the electric constant (dielectric permittivity of vacuum) and $\mu_{0}=12.57(4 \pi) \cdot 10^{-7} \mathrm{Vs} / \mathrm{Am}$ is the magnetic constant (magnetic permeability of vacuum). For the 1DWG we can simplify these equations taking into account that there is no variation in $y$-direction: $\partial / \partial y=0$.

\footnotetext{
${ }^{1}$ This leads to the 'easy-to-remember' relation $\lambda[\AA] \simeq 12.4 / E[\mathrm{keV}]$
} 
Modes in planar waveguides can be classified in TE (transverse electric) and TM (transverse magnetic) modes (Marcuse 1974; Pfeiffer. 2002) - TE-modes do not have a component of the electric field in the direction of wave propagation while TM modes have no longitudinal magnetic field component. In our analysis we will focus on TE modes, corresponding to our experiments. The difference between TE and TM modes for incidence angles well below one degree however is very small.

\subsubsection{Transverse electric modes}

For the description of the electric field in a $1 D W G$, we will regard time harmonic fields $\left(\Psi=E_{i}\right.$ or $H_{i}$ with $\left.i=x, y, z\right)$ with a time dependance

$$
\Psi \sim e^{i \omega t}
$$

where the radiation frequency $\omega$ is related to the frequency $f$ by $\omega=2 \pi f$. The $\mathrm{x}$-dependance is given by

$$
\Psi \sim e^{-i \beta x}
$$

where $\beta$ is the projection $k_{x}$ of the wavevector $k=2 \pi / \lambda$. In the literature $\beta$ is known as the propagation constant. Therefore we have

$$
\Psi=\Psi(z) e^{i(\omega t-\beta x)}
$$

For further treatment we will omit this factor which is common to all field quantities. With the nonzero field components $E_{y}, H_{x}$ and $H_{z}$ and with $E_{z}=0$, we get from Eqs. 3.3, 3.4 and 3.7

$$
\begin{gathered}
-i \beta H_{z}-\frac{\partial H_{x}}{\partial z}=i \omega \epsilon_{0} n^{2} E_{y} \\
i \beta E_{y}=-i \omega \mu_{0} H_{z} \\
\frac{\partial E_{y}}{\partial z}=-i \omega \mu_{0} H_{x}
\end{gathered}
$$

from which we find the $H$-components

$$
H_{z}=-\frac{i}{\omega \mu_{0}} \frac{\partial E_{y}}{\partial x}=-\frac{\beta}{\omega \mu_{0}} E_{y}
$$

and

$$
\begin{aligned}
H_{x}= & \frac{i}{\omega \mu_{0}} \frac{\partial E_{y}}{\partial z} \\
& -19-
\end{aligned}
$$


These two equations can now be substituted in Eq. 3.8, leading to the one dimensional reduced wave equitation (Helmholtz equation):

$$
\frac{\partial^{2} E_{y}}{\partial z^{2}}+\left(n^{2} k^{2}-\beta^{2}\right) E_{y}=0
$$

where $k=\omega \sqrt{\epsilon_{0} \mu_{0}}=2 \pi / \lambda$. Eq. 3.13 can be solved by the Ansatz

$$
E_{y}(z)= \begin{cases}A e^{\gamma z} & \text { for } z<0 \\ B \cos (\kappa z)+C \sin (\kappa z) & \text { for } 0 \leq z \leq d \\ D e^{-\gamma(z-d)} & \text { for } d<z\end{cases}
$$

with

$$
\gamma^{2}=\beta^{2}-n_{2}^{2} k^{2}
$$

and

$$
\kappa^{2}=n_{1}^{2} k^{2}-\beta^{2} .
$$

Next we will consider the continuity of $E_{y}$ and $H_{x}$ (i.e. of $\partial E_{y} / \partial z$ ) which gives:

$$
A=B \text { and } D=B \cos (\kappa d)+C \sin (\kappa d)
$$

as well as

$$
\begin{array}{ccc}
A \gamma & = & C \kappa \\
-A \kappa \sin (\kappa d)+C \kappa \cos (\kappa d) & = & -A \gamma \cos (\kappa d)-C \gamma \sin (\kappa d)
\end{array}
$$

or equivalently

$$
\left(\begin{array}{cc}
\gamma & -\kappa \\
\gamma \cos (\kappa d)-\kappa \sin (\kappa d) & \kappa \cos (\kappa d)+\gamma \sin (\kappa d)
\end{array}\right)\left(\begin{array}{l}
A \\
C
\end{array}\right)=0
$$

which has a solution only if the determinant vanishes:

$$
\tan (\kappa d)=\underbrace{\frac{2 \kappa \gamma}{\kappa^{2}-\gamma^{2}}}_{=: \Gamma(\kappa d)}
$$

Solving this transcendental equation numerically, we get a fixed number $N$ of resonant modes at parameters $\kappa_{n}, n \in(0, N-1)$. $N$ only depends on $\rho_{1}, \rho_{2}$ and $d$, where $\rho_{i}$ is the energy independent scattering length density of each material, since with Eqs. 3.1, 3.15 and 3.16, $\gamma_{n}$ can be written as $\gamma=\sqrt{\left(4 \pi\left(\rho_{2}-\rho_{1}\right) r_{e}-\kappa_{n}^{2}\right)}$. This is especially important for the resonant beam coupling (RBC) scheme which will 


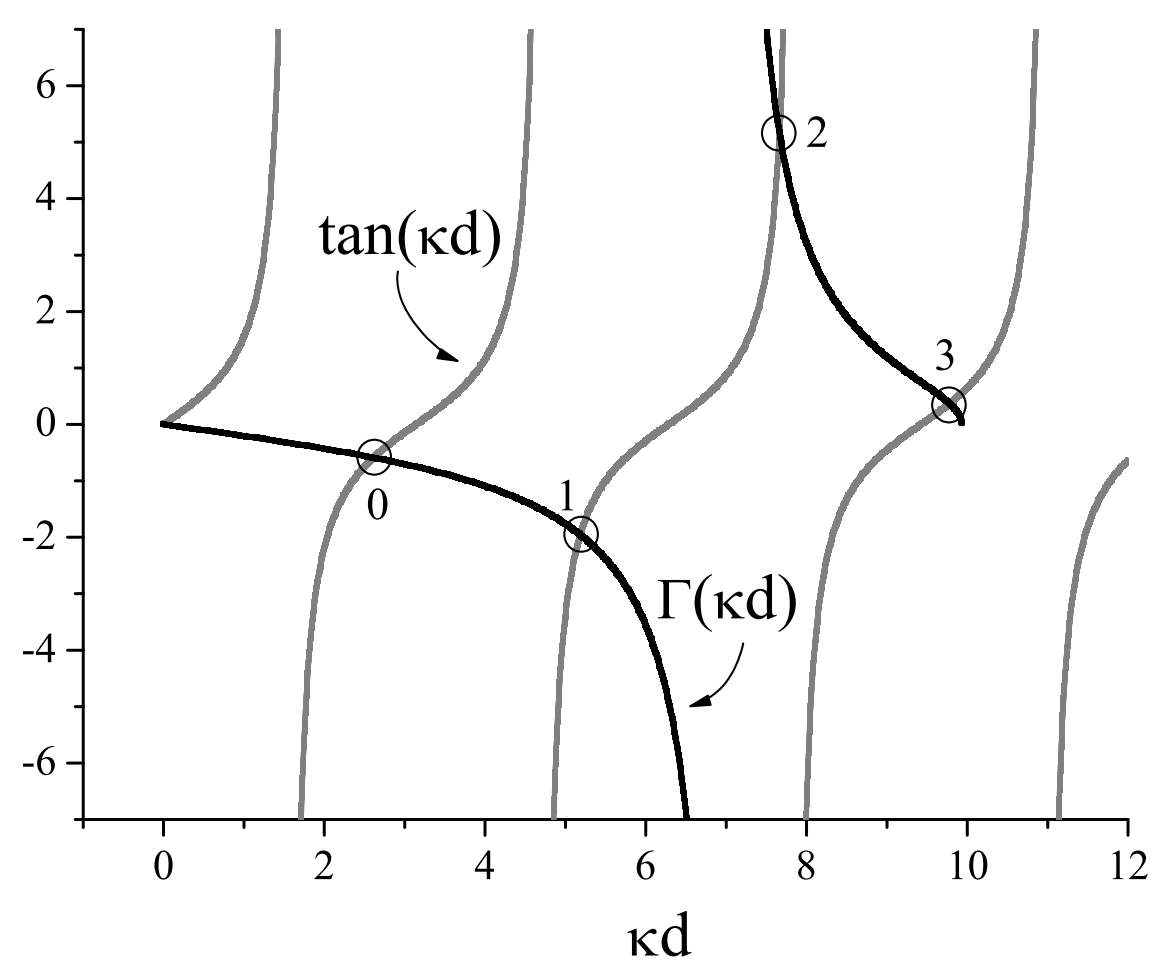

Figure 3.2: The 4 intersections of tan $(\kappa d)$ and $\Gamma(\kappa d)$ are solutions of the transcendental eigenvalue equation (Eq. 3.20) as calculated for a $50 \mathrm{~nm}$ thick waveguide layer with refractive index $n_{1}=1-0.5 \cdot 10^{-5}$ and a cladding with $n_{2}=1-1 \cdot 10^{-5}$.

be described in detail in section 3.3.1.

As an example we have plotted the two sides of Eq. 3.20 in Fig. 3.2 for an artificial waveguide with refractive indices $n_{1}=1-0.5 \cdot 10^{-5}$ and $n_{2}=1-1.0 \cdot 10^{-5}$ at an x-ray wavelength of $1 \AA$ and a guiding layer thickness $d=50 \mathrm{~nm}$. The intersections of the the black curve and the gray curve represent solutions of the transcendental equation. As we can see their total number $N$ is limited since for $\beta<n_{2} k, \gamma$ becomes imaginary.

$$
\gamma=0 \quad \text { or equivalently } \quad \beta=n_{2} k
$$

can therefore be regarded as the cutoff condition for mode propagation. The $\Gamma(\kappa d)$ curve ends at the point $\left(n_{1}^{2}-n_{2}^{2}\right)^{1 / 2} k d=\kappa d$. Introducing the dimensionless Potential $V:=\left(n_{1}^{2}-n_{2}^{2}\right)^{1 / 2} k d$ we find at the cutoff point the relation $V_{c}=(\kappa d)_{c}$ and with Eqs. 3.15, 3.16, 3.20 and 3.21:

$$
V_{c}=\nu \pi, \nu=0,1,2, \ldots
$$



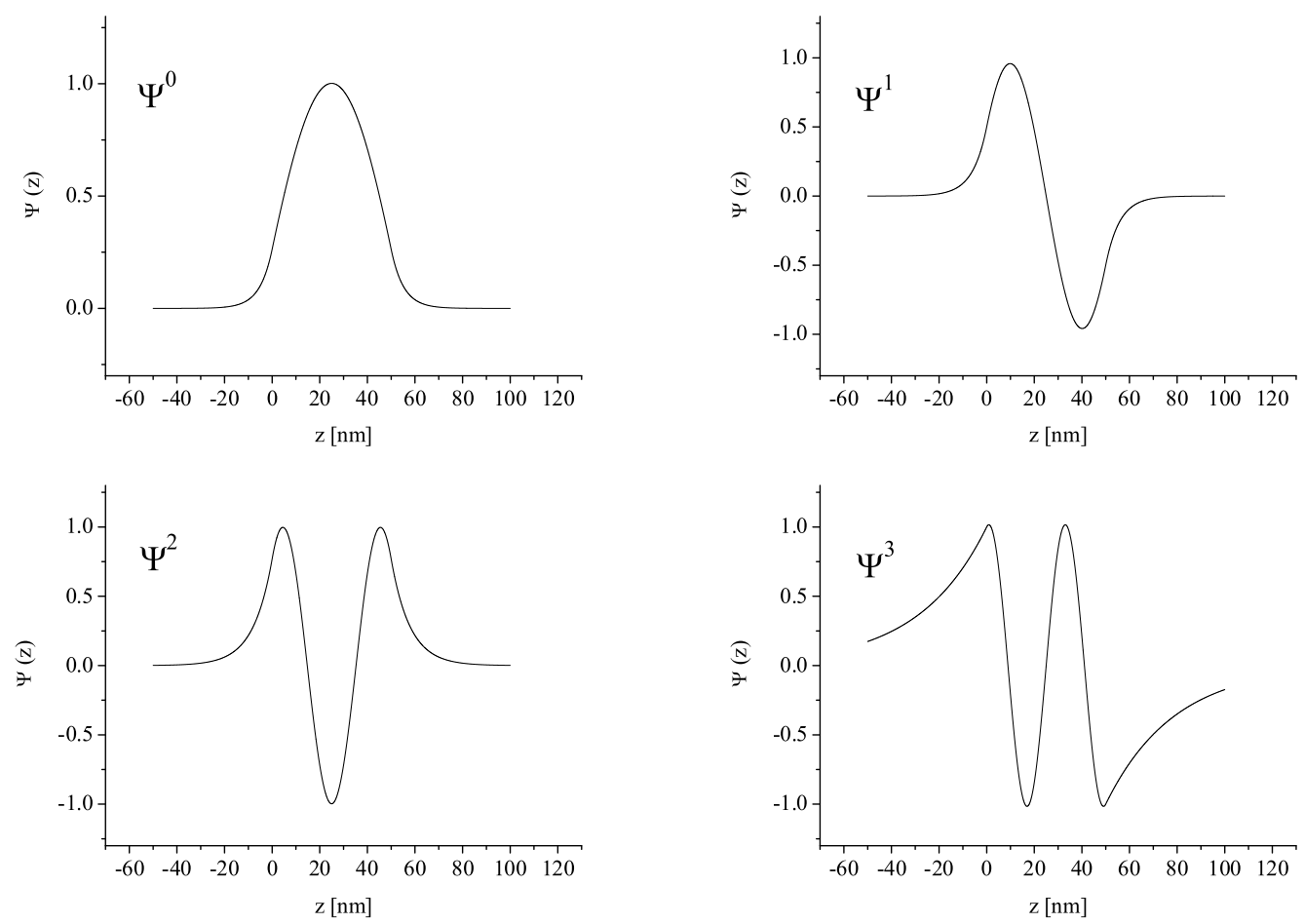

Figure 3.3: Calculated normalized internal field distribution $\Psi^{j}$ of a planar waveguide (refractive indices $n_{1}=1-0.5 \cdot 10^{-5}$ and $n_{2}=1-1 \cdot 10^{-5}$ for the four supported modes $(j \in[0,1,2,3]$, see Fig. 3.2). guiding layer: $50 \mathrm{~nm}, \lambda=1 \AA$.

The restriction $V<N \pi$ then gives the total number $N$ of supported modes: $N=$ $[V / \pi]_{i n t}$, where int defines the next integer lager than $V / \pi$.

The field distribution in the waveguide is given by Eq. 3.14, while three of the parameters $A, B, C$, and $D$ are given by Eqs. 3.17 and 3.18. One parameter is free and scales with the field amplitude or correspondingly the intensity. Both, the normalized electric field and the normalized intensity distribution in the waveguide described above are plotted in Figs. 3.3 and 3.4, respectively. These plots also demonstrate, that the intensity is mainly restricted to the area of the guiding core between $z=0 \mathrm{~nm}$ and $z=50 \mathrm{~nm}$. From the solutions of Eq. 3.20 together with Eq. 3.16 and the incidence angle $\alpha_{i}$ we find for the resonant beam coupling scheme (section 3.3.1) the useful relation

$$
\begin{aligned}
\kappa_{n}= & \frac{2 \pi}{h c} E \sin \alpha_{i n t} \\
& -22-
\end{aligned}
$$



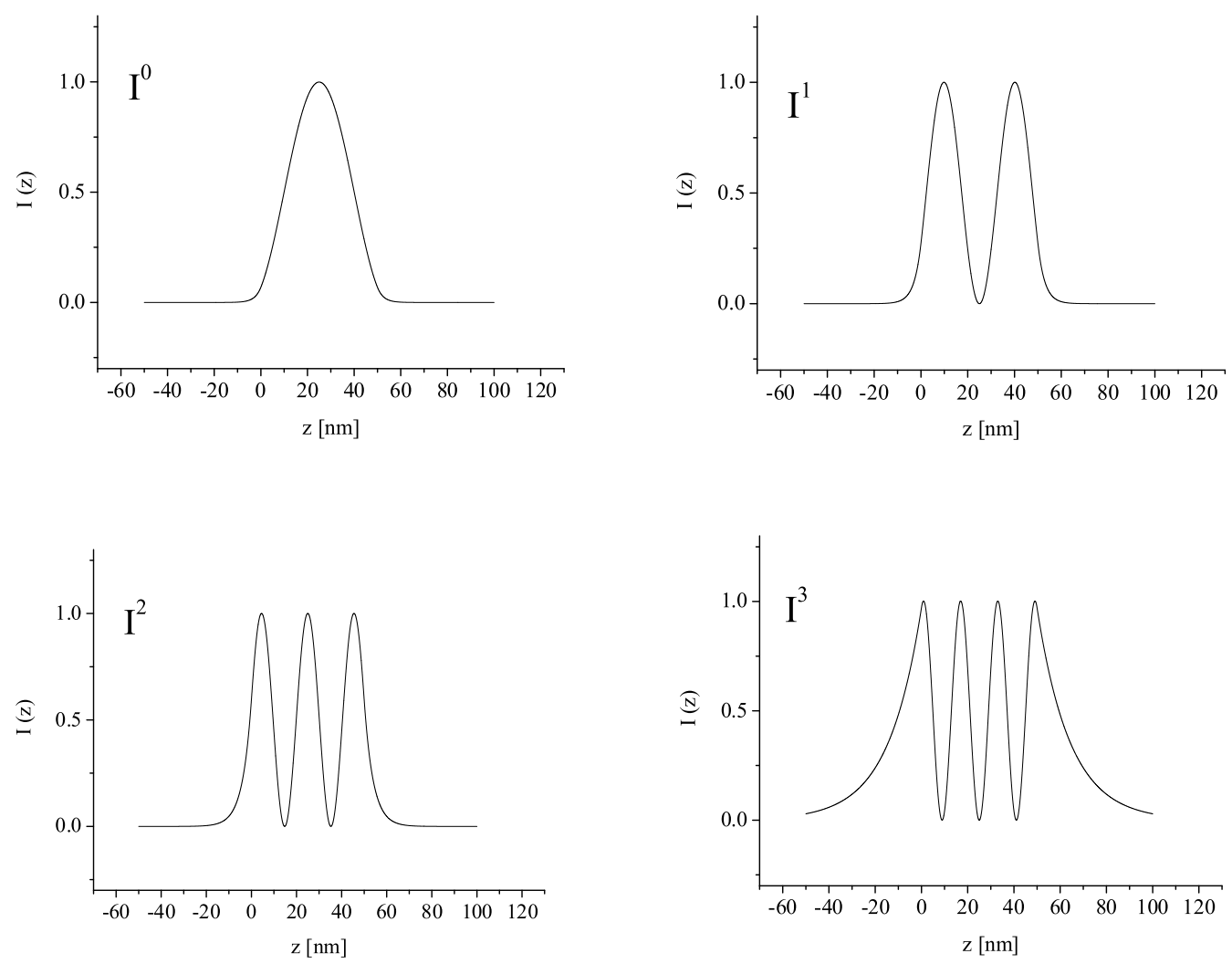

Figure 3.4: Calculated normalized internal intensity distribution $I^{j}$ of the waveguide described in Fig. 3.3.

which intimately links the resonant energies and the incidence angle of the impinging x-ray beam. Here, $\alpha_{i n t}$ is the refraction corrected internal propagation angle, $\alpha_{i n t}^{2}=\alpha_{i}^{2}-\alpha_{c}^{2}$ with the critical angle $\alpha_{c}$ of the guiding layer.

\subsubsection{Limits of x-ray nano-beam production}

Let us now consider a waveguide with an air gap which becomes more and more narrow as the beam propagates in it (see Fig. 3.5). Eq. 3.20 is then best solved in polar coordinates $r$ and $\phi$ with $r=\sqrt{x^{2}+z^{2}}$ and $\phi=-\arctan (z / x)$. Assuming that the beam does not penetrate into the confining material we find for the $m^{\text {th }}$ Eigenmode (Bergemann et al. 2003):

$$
u_{m}(r, \phi)=H_{m \pi / \phi_{0}}^{(1)}(k r) \sin \left(\frac{m \pi \phi}{\phi_{0}}\right)
$$




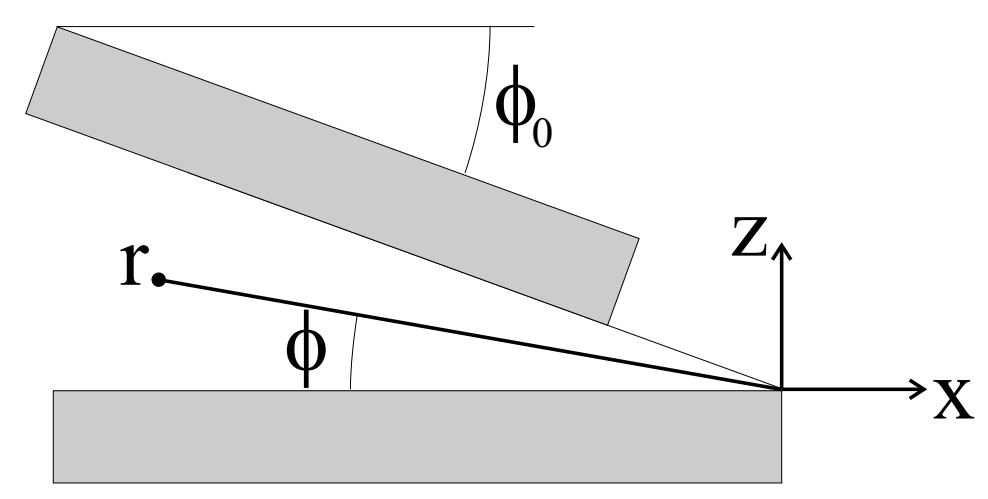

Figure 3.5: Sketch of a tapered waveguide wedge.

where $H_{m \pi / \phi}^{(1)}$ is the Hankel function of the first kind of fractional order $m \pi / \phi_{0} \sim 10^{5}$ which can be approximated by the asymptotic expansion (Abramowitz and Stegun 1965)

$$
H_{m \pi / \phi_{0}}^{(1)}(k r) \simeq \sqrt{\frac{2}{\pi k r}} e^{i\left(k r-\frac{m \pi^{2}}{2 \phi_{0}}-\frac{\pi}{4}-\frac{m^{2} \pi^{2}}{\left.2 \phi_{0}^{2 k r}\right)}\right.}
$$

since $k r$ is large $\left(\sim 10^{8}\right)$. In reality, the field in the confining material is nonzero: $u \sim e^{i n k r}$ with the refractive index $n=1-\delta . \delta$ can be expressed in terms of the critical angle $\Theta_{c}$ of total reflection (Als-Nielsen 2001) or by Eq. 3.1. From the phase in Eq. 3.24 given by Eq. 3.25 we can define an effective wavevector $k_{\text {eff }}$ as

$$
k_{e f f}=k-\frac{m^{2} \pi^{2}}{2 \phi_{0}^{2} k r^{2}} .
$$

If this becomes equal to the wavevector $k(1-\delta)$ in the medium, the wave starts to leak out of the guide which happens at $m$ times the critical gap width

$$
W_{c}=\frac{\lambda}{2 \theta_{c}}=\frac{1}{2} \sqrt{\frac{\pi}{r_{e} n_{e}}}
$$

with the electron density $n_{e}$ of the confining material. $W_{C}$ represents a lower limit for waveguide nanobeams which is in the region of $\sim 8 \mathrm{~nm}$ for gold cladding layers to $\sim 20 \mathrm{~nm}$ for Si. It is currently a field of discussion if the limit is applicable to all diffractive, reflective and refractive focusing x-ray optics. As e.g. for FZPs the spot size is limited by the outermost zone width the above concept is also valid for this type of focusing tool. Schroer and Lengeler in turn suggest a design for specially 
shaped refractive lenses with a possible spot size of $2 \mathrm{~nm}$ (Schroer and Lengeler. 2005). 


\subsection{Two dimensionally confining waveguides}

So far we have restricted the description of waveguides to the planar case. However, in practice it is by far more interesting to produce a two dimensionally confined beam. Therefore we have to generalize the above concept to 2D-waveguides. According to the experimental results which are presented in chapter 6 , we will focus on rectangular geometries as sketched in Fig. 3.6. An exact analytical solution for

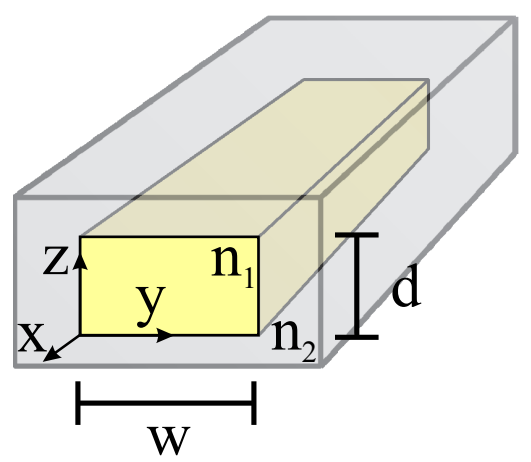

Figure 3.6: Sketch of a rectangular waveguide with the guiding core (refractive index $n_{1}$ ) and the surrounding cladding material $\left(n_{2}\right)$.

these geometries is not possible but analytical approximations can be made with high accuracy, mainly in the case of large core dimensions. In the following description we will follow an approach which is described in detail in (Marcuse 1974) where we assume that the field is mainly restricted to the guiding core of the device. Consequently we find only small contributions at the side of the core and even less in the 'corners', i.e. in the top left region, the top right region and so on. The contributions of these corner regions are neglected in the analytical examination described in the following. Since the contributions of the corner regions increase with decreasing core size a numerical approach is used to calculate the internal field distribution of small 2DWGs solving the Helmholtz equation in the approximation of the parabolic wave equation (see below).

In analogy to the one dimensional case the Helmholtz equation (3.20) for TE modes is given by:

$$
\begin{aligned}
\frac{\partial^{2} E_{y}(y, z)}{\partial y^{2}}+\frac{\partial^{2} E_{y}(y, z)}{\partial z^{2}} & +\left(n(y, z)^{2} k^{2}-\beta^{2}\right) E_{y}(y, z)=0 \\
& -26-
\end{aligned}
$$


with

$$
n(y, z)= \begin{cases}n_{1}, & \text { for } 0 \leq z \leq d \text { and } 0 \leq y \leq w \\ n_{2}, & \text { else }\end{cases}
$$

The following set of five equations satisfies the reduced wave equation and the boundary conditions. The factor 3.7 is again factored out for clarity. Inside the guide $(0 \leq y \leq w$ and $0 \leq z \leq d)$ we find:

$$
\Psi=\left(i / \kappa_{z} \beta\right)\left(n_{1}^{2} k_{0}^{2}-\kappa_{z}^{2}\right) \cos \left[\kappa_{y}(y+\eta)\right] \sin \left[\kappa_{z}(z-\xi)\right] .
$$

with phase parameters $\xi$ and $\eta$ which are determined by:

$$
\begin{gathered}
\tan \left(\kappa_{z} \xi\right)=-\frac{n_{2}^{2}}{n_{1}^{2}} \frac{\kappa_{z}}{\gamma_{z}} \\
\tan \left(\kappa_{y} \eta\right)=-\frac{\gamma_{y}}{\kappa_{y}}
\end{gathered}
$$

Outside the guiding core we find:

$$
\Psi=-i\left[\left(\gamma_{z}^{2}+n_{2}^{2} k_{0}^{2}\right) / \gamma_{z} \beta\right] \cos \left[\kappa_{y}(y+\eta)\right] \cos \left[\kappa_{z}(d-\xi)\right] \exp \left[-\gamma_{z}(z-d)\right]
$$

for $0 \leq y \leq w$ and $z<0$, i.e. below the core,

$$
\Psi=i\left[\left(\gamma_{z}^{2}+n_{2}^{2} k_{0}^{2}\right) / \gamma_{z} \beta\right] \cos \left[\kappa_{y}(y+\eta)\right] \cos \left(\kappa_{z} \xi\right) \exp \left(\gamma_{z} z\right)
$$

for $0 \leq y \leq w$ and $d<z$, i.e. above the core,

$$
\Psi=i\left(n_{1}^{2} / n_{2}^{2}\right)\left[\left(n_{2}^{2} k_{0}^{2}-\kappa_{z}^{2}\right) / \kappa_{z} \beta\right] \cos \left[\kappa_{y}(b+\eta)\right] \exp \left[-\gamma_{y}(y-b)\right] \sin \left[\kappa_{z}(z-\xi)\right],
$$

for $w<y$ and $0 \leq z \leq d$, i.e. to the right of the core, and

$$
\Psi=i\left(n_{1}^{2} / n_{2}^{2}\right)\left[\left(n_{2}^{2} k_{0}^{2}-\kappa_{z}^{2}\right) / \kappa_{z} \beta\right] \cos \left(\kappa_{y} \eta\right) \sin \left[\kappa_{z}(z-\xi)\right] \exp \left(\gamma_{y} y\right) .
$$

for $y<0$ and $0 \leq z \leq d$, i.e. to the left of the core.

For $\kappa_{y}, \kappa_{z}, \gamma_{y}, \gamma_{z}$, and $\beta$ we find:

$$
\gamma_{y}^{2}=\left(n_{1}^{2}-n_{2}^{2}\right) k_{0}^{2}-\kappa_{y}^{2}, \quad \gamma_{z}^{2}=\left(n_{1}^{2}-n_{2}^{2}\right) k_{0}^{2}-\kappa_{z}^{2},
$$

and

$$
n_{1}^{2} k_{0}^{2}+\beta^{2}=\kappa_{y}^{2}+\kappa_{z}^{2}
$$


Together with the boundary conditions we get a set of two transcendental eigenvalue equations: For $\kappa_{y}$ we have

$$
\tan \left(\kappa_{y} w\right)=\frac{2 \kappa_{y} \gamma_{y}}{\kappa_{y}^{2}-\gamma_{y}^{2}}
$$

Equation 3.39 is already known from the TE modes for a planar waveguide.

The corresponding expression for $\kappa_{z}$ is:

$$
\tan \left(\kappa_{z} d\right)=\frac{2 \kappa_{z} \gamma_{z}}{\frac{n_{2}^{2}}{n_{1}^{2}} \kappa_{z}^{2}-\frac{n_{1}^{2}}{n_{2}^{2}} \gamma_{z}^{2}}
$$

This is the transcendental equation which is found for TM modes of the planar waveguide. Note, that since $n_{1}$ and $n_{2}$ are both $\approx 1$, the difference between TE and TM modes is very small. We can see that two dimensional waveguides do also support a limited number of modes and, similar to the one dimensional case, this number is wavelength independent. With the two transcendental eigenvalue equations providing $\mathrm{N}$ and $\mathrm{M}$ solutions for $\kappa_{y}$ and $\kappa_{z}$ respectively, the total number of solutions is $N \times M$. In the notation of the respective modes this is represented by two indices: $\Psi^{p q}$ with $p \in(0, N-1)$ and $q \in(0, M-1)$. As an example in Fig. 3.7 we have plotted the internal intensity distribution $\left|\Psi^{p q}\right|^{2}$ in a rectangular waveguide (side length: $50 \times 50 \mathrm{~nm}^{2}$, refractive indices $n_{1}=1-0.5 \cdot 10^{-5}$ and $n_{2}=1-1.0 \cdot 10^{-5}$ at an x-ray wavelength $\lambda=1 \AA$ ) for the $\Psi^{00}$ to $\Psi^{22}$ modes. In the resonant beam coupling scheme with monochromatic illumination, these modes are excited separately by changing the incidence angle of the impinging x-ray beam, so we find only one of the shown examples at a time. In the front coupling regime, all supported modes can be excited at the same time (see sect. 3.3.2) and inside the waveguide they will mix. The internal field distributions of all excited modes are therefore added and the intensity distribution then corresponds to the square of this sum (see section 3.4). 

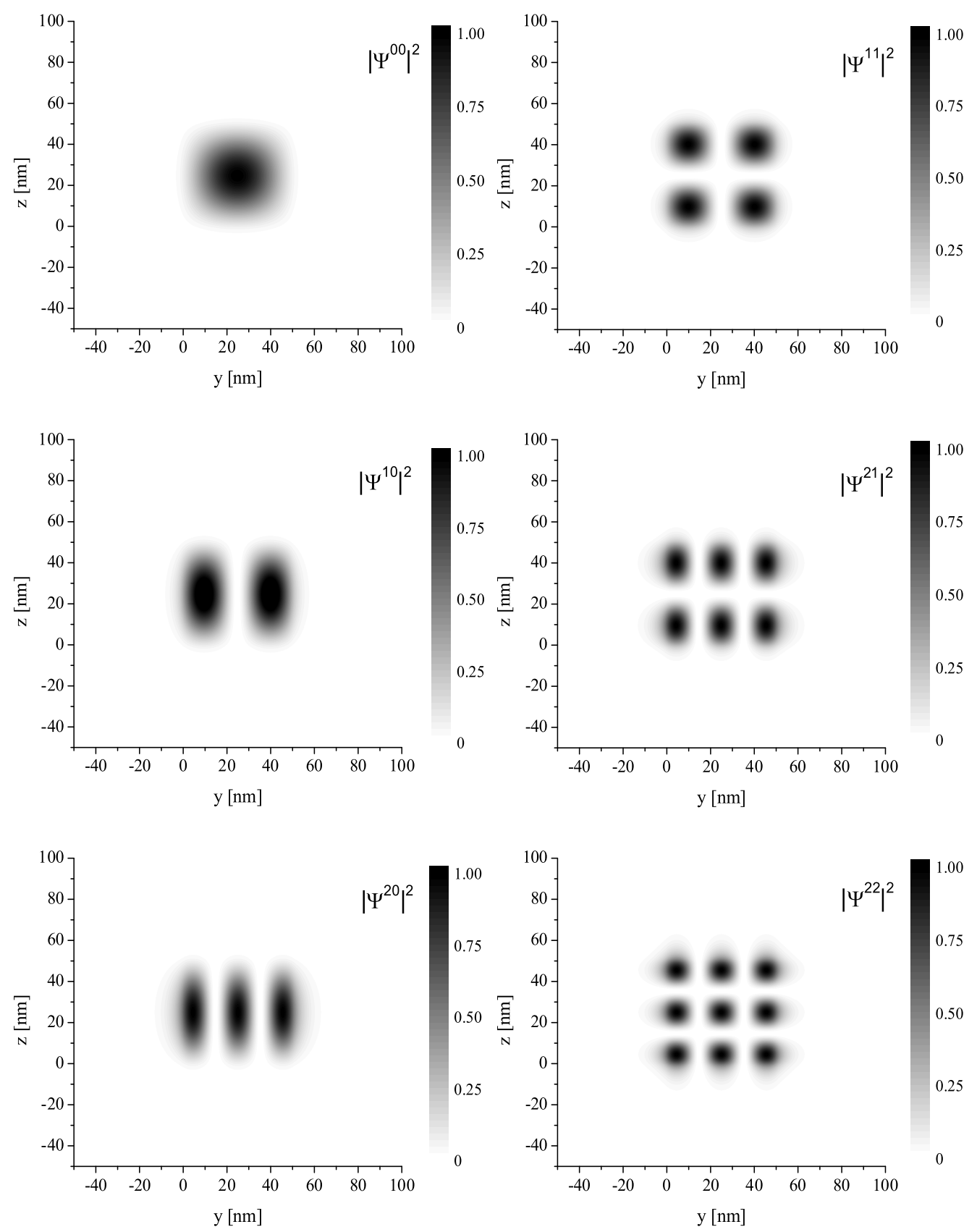

Figure 3.7: Sketch of the normalized internal intensity distribution $\left|\Psi^{p q}\right|^{2}$ of a rectangular $50 \mathrm{~nm} \times 50 \mathrm{~nm}$ waveguide (refractive indices $n_{1}=1-0.5 \cdot 10^{-5}$ and $n_{2}=1-1.0 \cdot 10^{-5}$ ) at an $x$-ray wavelength $\lambda=1 \AA$. 


\subsection{Coupling schemes for hard x-rays}

As the guiding core $(d<100 \mathrm{~nm})$ of an $x$-ray waveguide is orders of magnitude smaller than a typical $x$-ray beam set e.g. by slits or pinholes $(d>5 \mu \mathrm{m})$, we can't just shine a plane wave onto its entrance, since the coupling efficiency would be far to low then. Different attempts were performed to couple the beam into the waveguide with enhanced efficiency, as there are e.g. coupling of a standing wave field into the guide (Zwanenburg et al. 1999), coupling via a thin top cladding layer (resonant beam coupling, RBC) (Spiller and Segmüller 1974; Feng et al. 1993; Di Fonzo et al. 2000; Salditt et al. 2003; Jarre et al. 2003) or coupling of a prefocused beam (Fuhse et al. 2004; Jarre et al. 2005). In the following we will focus on the latter two cases which correspond to our experiments.

\subsubsection{Resonant Beam Coupling - RBC}

Coupling a hard x-ray beam into a waveguide via a thin cladding layer has been examined since the first experiments of Spiller et al. in 1974. A variety of experiments has been performed with such devices, mainly to study one dimensional beam compression. On the following pages we will discuss the fundamental parameters involved in the characterization of efficient RBC waveguides. Therefore we will make use of a powerful X-ray analysis tool, namely the measurement of the samples reflectivity. The geometry of such a measurement is sketched in Fig. 3.8 a): The reflectance is the measured intensity of an x-ray beam impinging at grazing incidence angle $\alpha_{i}$ measured at the exit angle $\alpha_{f}$ restricted to the case that these two angles are both the same $\left(\alpha_{i}=\alpha_{f}\right)$, see e.g. (Als-Nielsen 2001). Now starting from the well known (Fresnel-) reflectance of e.g. a Ni- substrate, we find that due to the refractive index decrement $\delta_{M}$ of the respective material $\mathrm{M}$ (here $\mathrm{Ni}$ ), the impinging beam is totally reflected for incidence angles $\alpha_{i}$ smaller than the critical angle $\alpha_{c}=\sqrt{2 \delta_{M}}$ leading to the so-called plateau of total (external) reflection which can clearly be identified in Fig. $3.8 \mathrm{~b}$ ) where we find the critical angle of $\mathrm{Ni}$ for a $12 \mathrm{keV}$ x-ray beam at approximately $0.28^{\circ}\left(\delta \simeq 1.23 \cdot 10^{-5}\right)$, represented by the vertical line in the graph. Yet we know from basic x-ray physics, that an evanescent wave field penetrates into the reflecting material (Als-Nielsen 2001). We will make use of this effect by introducing an additional layer of a second material a few $\mathrm{nm}$ below the surface. The second material is chosen such that its refractive index decrement is smaller than the one of the originally used material. As an example 

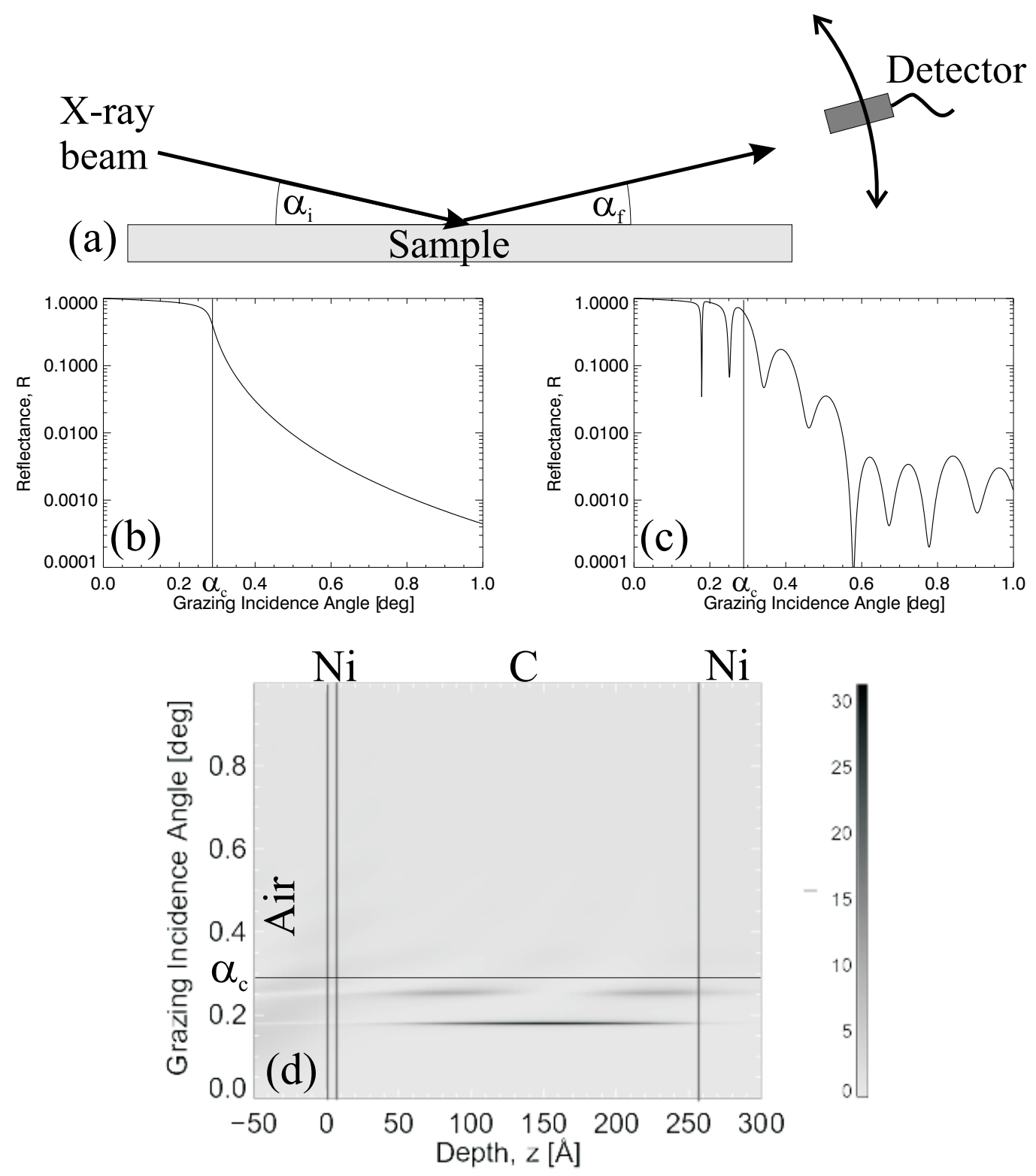

Figure 3.8: a) Sketch of an $x$-ray reflectivity $\left(\alpha_{i}=\alpha_{f}\right)$ measurement b) Fresnel reflectivity of blank $\mathrm{Ni}$ wafer at $\mathrm{E}=12 \mathrm{keV}$ and c) reflectivity of a $\mathrm{Ni} / \mathrm{C}(25 \mathrm{~nm}) / \mathrm{Ni}(5 \mathrm{~nm})$ layer system with its characteristic cusps in the plateau of total reflection indicative for coupling of the impinging beam into the guide. (d) The calculation of the internal intensity distribution as a function of the incidence angle exhibits internal intensity enhancement at the (angular) position of the cusps in (c).

we chose to introduce a $25 \mathrm{~nm}$ thick carbon layer $\left(\delta \simeq 3.28 \cdot 10^{-6}\right.$ at $\left.12 \mathrm{keV}\right)$ only $5 \mathrm{~nm}$ below the Ni surface. The reflectance of this device (Fig. $3.8 \mathrm{c}$ ) is of course 
entirely different from that of the blank Ni-substrate (Fig. 3.8 b). Apart from the eye-catching, but for the purpose of this thesis unimportant interference pattern at higher incidence angles $\left(\alpha_{i}>\alpha_{c}\right)$, we find that at certain incidence angles below the critical angle, i.e. on the plateau of total reflectance where $\alpha_{i} \leq \alpha_{c}$, the reflectance breaks in dramatically. Calculating the internal intensity distribution as a function of the incidence angle shows that at these angular positions the internal intensity distribution of this device is enhanced by a factor of 30; the beam is coupled into the carbon layer. The calculations presented above and in the following were performed using the freely available imd software package with the implemented algorithms described in (Windt. 1998). As an example in Fig. 3.9 we have plotted the reflectivity of four $\mathrm{Ni} / \mathrm{C} / \mathrm{Ni}$ waveguides with all other parameters kept constant except the guiding layer thickness. While a $10 \mathrm{~nm}$ guiding layer supports one mode only (monomodal WG), we find two modes for a $20 \mathrm{~nm}$ guide, and three modes for the $32 \mathrm{~nm}$ carbon layer. Thicker guiding layers can support a lot of modes leading to a comb like profile in the reflectance with the cusps getting closer and closer to each other. Their experimental detection therefore requires high angular (or energetic, see chapter 5) resolution.

To achieve the most efficient coupling of the beam we have to find the optimum cladding layer parameters. On the one hand, the guiding layer thickness defines how much of the incident beam can couple into the guide - naively spoken: the thicker the cladding the less intensity will be transmitted. On the other hand it also controls how much of the guided beam can leak from the guiding layer - the thinner the cladding layer the more leaking of the guided beam will occur. In Fig. 3.10 the effect of different top cladding layer thicknesses is shown again for $\mathrm{Ni} / \mathrm{C} / \mathrm{Ni}$ waveguides at $12 \mathrm{keV}$. Corresponding to the different depths of the cusps in the plateau of total reflection which are indicative of coupling of the x-ray beam $(\mathrm{a}-\mathrm{c})$ we find that the internal intensity enhancement - here represented by the intensity in the center of the guiding layer - is highly sensitive on this parameter $(d)$. Therefore, the guiding layer has to be considered as a very important design parameter. Generally spoken we find that higher $\mathrm{Z}$ elements require thinner cladding layers than lower $\mathrm{Z}$ elements. The choice of the materials is discussed next: With $\kappa$ and $\gamma$ in Eq. 3.20 being functions of the refractive indices of the waveguide materials the number of supported modes or equivalently the number of solutions to the transcendental eigenvalue equation depends on these parameters. Fig. 3.11 demonstrates this. A guiding layer thickness of $22 \mathrm{~nm}$ results in only one supported mode 

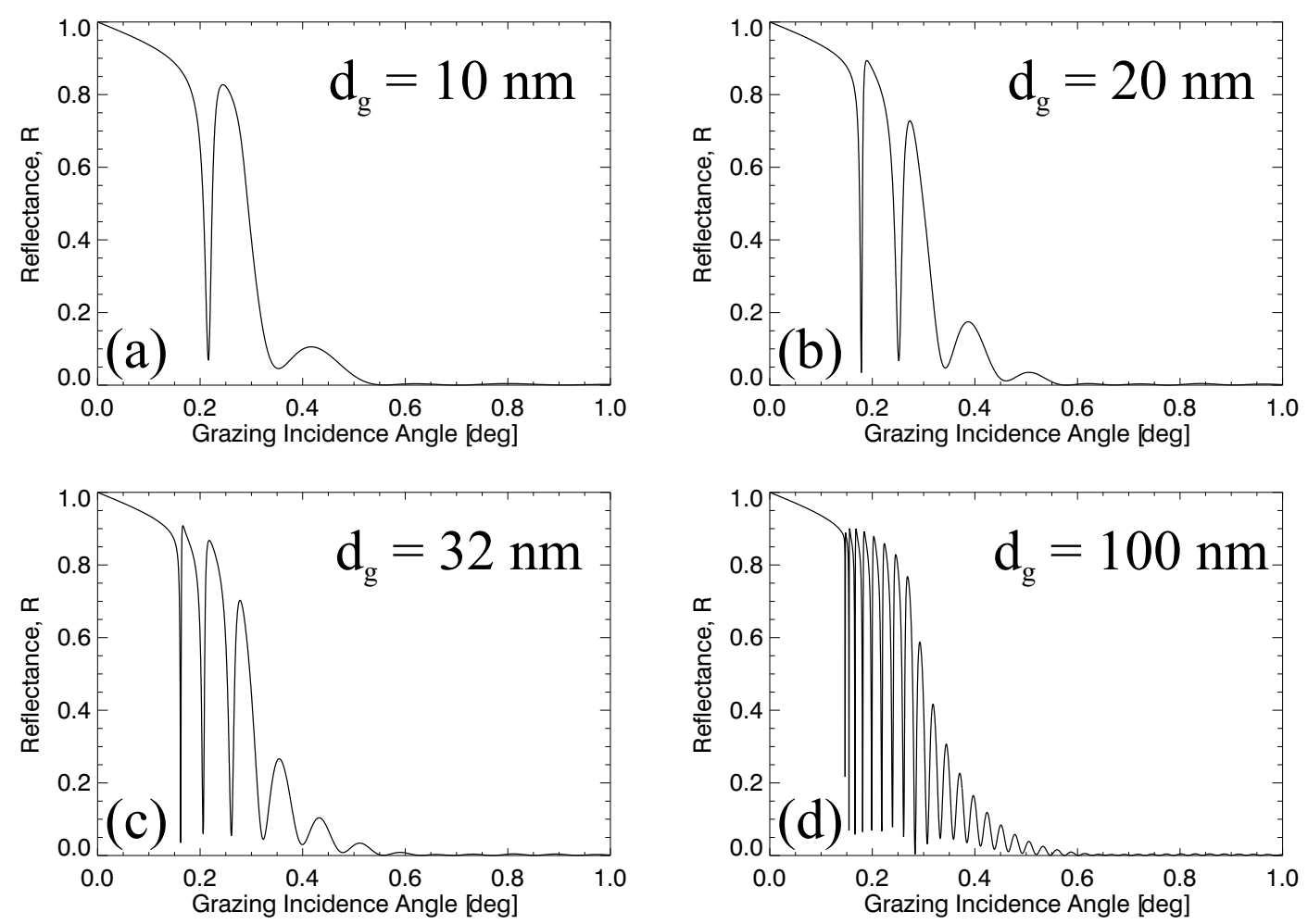

Figure 3.9: Reflectance of $N i / C / N i$ - RBC waveguides with different guiding layer thicknesses $d_{g}$ and constant top cladding layer thickness $d_{c}=5 \mathrm{~nm}$. The total number of supported modes for a given combination of materials is adjusted only by the waveguide geometry. The calculations were performed for an X-ray energy of $E=12.0 \mathrm{keV}$.

for a $\mathrm{Si} / \mathrm{PMMA}^{2} / \mathrm{Si}$ waveguide i.e. it is monomodal (Fig. 3.11 a). In the case of a $\mathrm{Ni} / \mathrm{C} / \mathrm{Ni}$ waveguide (Fig. 3.11 b) already two modes are supported for otherwise identical relevant waveguide parameters. With the comparatively large refractive index decrement difference between gold and air, the $\mathrm{Au} / \mathrm{Air} / \mathrm{Au}$ waveguide even supports three modes (Fig. $3.11 \mathrm{c}$ ). Please note that the cladding layer thickness in these examples is not the same in all three cases but was adapted to maximum coupling efficiency in order to emphasize the cusps for better comparison.

The resonant beam coupling scheme has so far only beam applied to monochromatic illumination of the waveguide. It was found that the excitation of each mode $\left(\Psi^{0}, \Psi^{1}, \ldots\right)$ corresponds to illumination at defined incidence angles $\alpha_{i}$. Applying

\footnotetext{
${ }^{2}$ Polymethyl methacrylate
} 

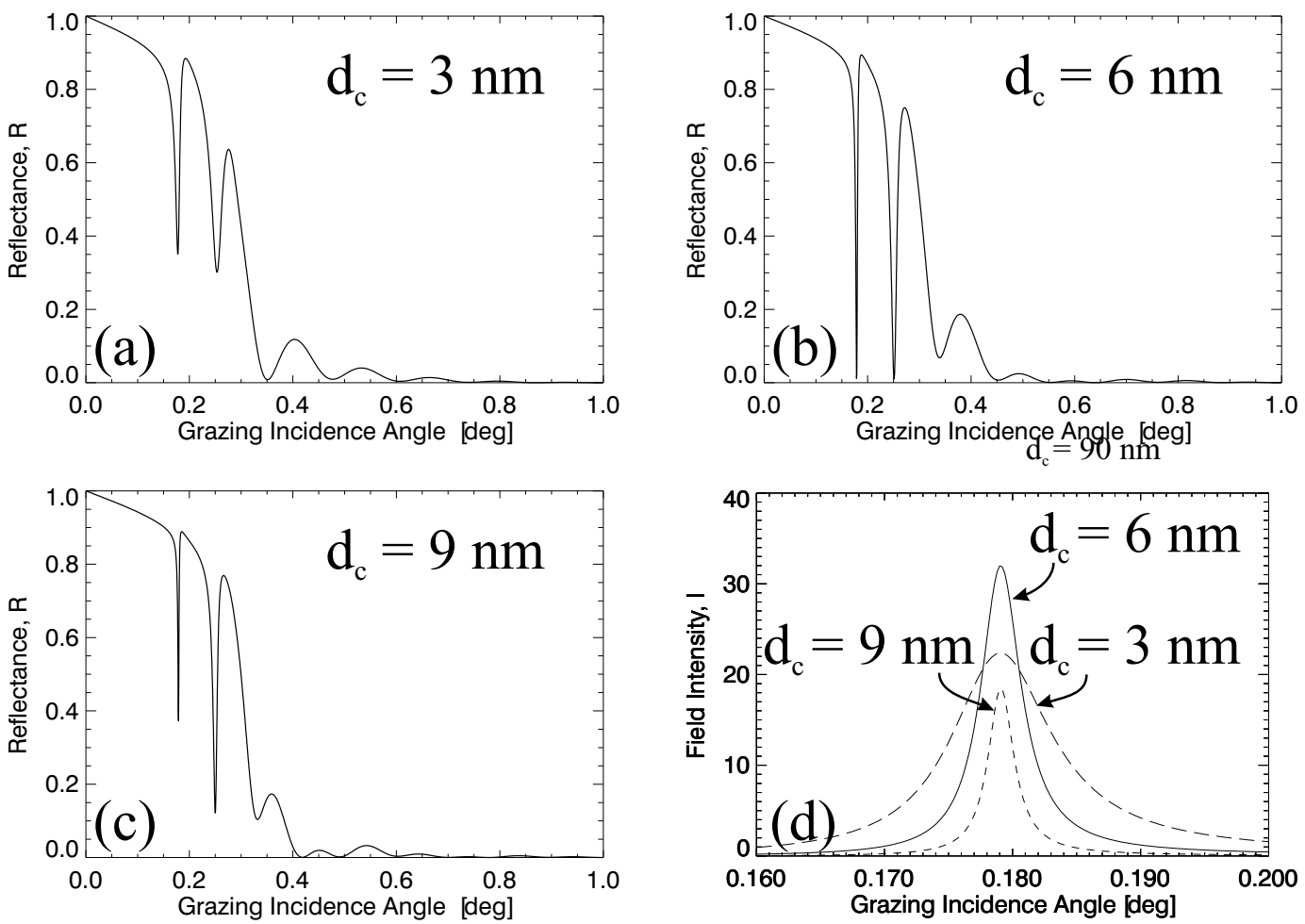

Figure 3.10: a) - c) Reflectance of $N i / C / N i$ - RBC waveguides with different top cladding layer thicknesses $d_{c}$ and constant guiding layer thickness $d_{g}=20 \mathrm{~nm}$. The coupling efficiency of a RBC waveguide or equivalently the depth of the cusps in the plateau of total reflection strongly depends on the thickness of this cladding layer, as can be seen in (d). Here the the maximum intensity in the center of the guiding layer is plotted for the three different cladding layer thicknesses (3, 6, $9 \mathrm{~nm}$ ). The calculations were performed for an X-ray energy of $E=12.0 \mathrm{keV}$.

this concept to white beam illumination eliminates this angular separation. With $\kappa$ and $\gamma$ both being functions of $\lambda$ we find that at any given incidence angle, the white beam provides all energies that fulfil the requirements of the transcendental eigenvalue equation. Therefore all modes will be excited at the same time now separated not by the incidence angle, but energetically. Please note that nonetheless, the total number of supported modes is fixed, given only by the waveguide geometry and the utilized materials (see e.g. Fig. 5.5). The characteristics of white beam illumination are subject of chapter 5 , where the experimental observations are discussed in more detail. 

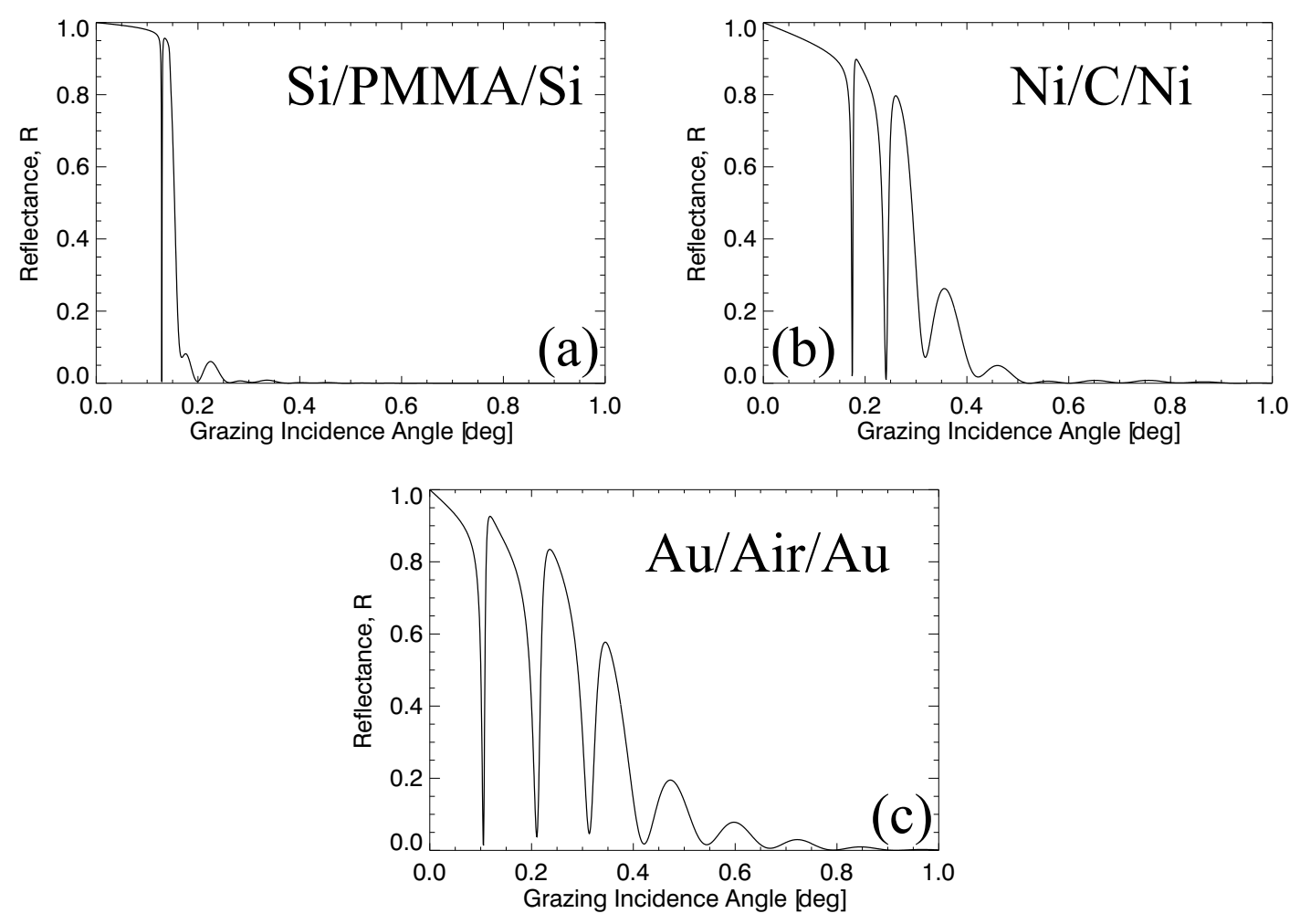

Figure 3.11: Reflectance of three $R B C$ waveguides with identical guiding layer thicknesses $d_{g}=22 \mathrm{~nm}$ but made from different materials. The different refractive index differences between the utilized materials lead to different numbers of supported modes for each material combination. The calculations were performed for an X-ray energy of $E=12.0$ $\mathrm{keV}$. The top cladding layer thickness of the three waveguides was adapted to maximum coupling efficiency resulting in a) $\left.d_{c}=25 \mathrm{~nm}, b\right) d_{c}=6 \mathrm{~nm}$ and c) $d_{c}=3 \mathrm{~nm}$. 


\subsubsection{Front Coupling}

As we have seen in section 3.1 the field distribution $\Psi(x, z)$ in a planar waveguide can be calculated solving the Helmholtz equation (Eq. 3.20) leading to a certain number of supported modes. Contrary to the RBC scheme with monochromatic illumination, in the front coupling scheme (see Fig. 3.12) multiple modes may be excited at the same time. The field distribution in the device is then given as a linear superposition of the propagating modes:

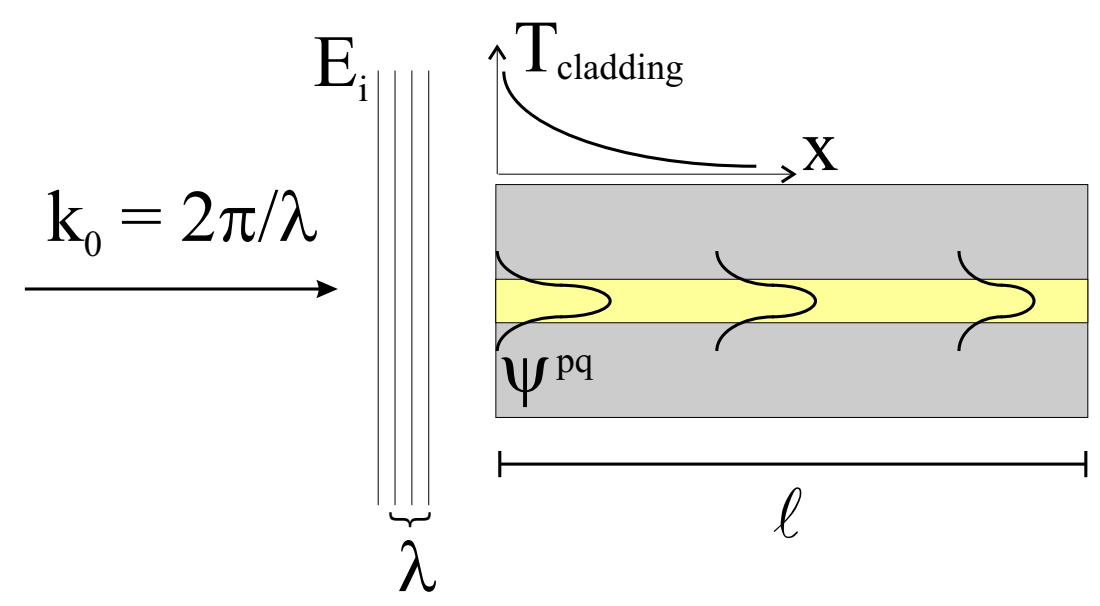

Figure 3.12: Sketch of a front coupling waveguide device. The incident beam excites modes $\psi^{p q}$ in the guiding core. Outside the guiding region, the beam is strongly damped by the cladding layer, while absorption of the guided modes in the core is by far less.

$$
\Psi(x, z)=\sum_{m} c_{m} \Psi_{m}(z) e^{-\beta_{m} x}
$$

with

$$
\begin{array}{clc}
A_{m} e^{\gamma_{m} z} & \text { for } & z<0 \\
\Psi_{m}(z)=B_{m} \cos \left(\kappa_{m} z\right)+C_{m} \sin \left(\kappa_{m} z\right) & \text { for } 0 \leq z \leq d \\
D_{m} e^{-\gamma_{m}(z-d)} & \text { for } \quad d<z
\end{array}
$$

The calculation of the respective parameters A to D follows the description given in section 3.1 while the coefficients $c_{m}$ are given by the projection of the incident field $E_{i}$ on the respective modes:

$$
c_{m}=\frac{1}{\left\|\Psi_{m}\right\|^{2}} \int E_{i}(z) \Psi_{m}(z) d z
$$


As the integral in Eq. 3.43 goes from $-\infty$ to $+\infty, c_{m}$ is zero for odd (asymmetric) modes in the case of symmetric illumination $E_{i}(z)$. Asymmetric illumination, e.g. caused by a plane wave impinging at an incidence angle other than perpendicular to the entrance surface thus changes $c_{m}$ and correspondingly the internal intensity distribution. In particular, Eq. 3.43 explains the sensitivity of the internal intensity distribution and consequently of the farfield distribution (see below) of multimodal waveguides on the incidence angle.

\section{The parabolic wave equation}

For many waveguides geometries other than circular ones the Helmholtz equation cannot be solved analytically. In order to approximate the solution numerically one can make use of numerical calculations solving the helmholtz equation in the approximation of the parabolic wave equation (Kopylov et al. 1995):

$$
2 i k_{0} \frac{\partial u}{\partial x}+\frac{\partial^{2} u}{\partial y^{2}}+\frac{\partial^{2} u}{\partial z^{2}}+k_{0}^{2}\left(n^{2}-1\right) u=0
$$

where $\mathrm{u}$ is defined by $\Psi(x, y, z)=u(x, y, z) e^{-i k_{0} x}$. The parabolic wave equation can be solved by finite-difference calculations (Fuhse and Salditt. 2004) calculating the field distribution step by step over the waveguide length. For comparison with experimentally obtained results the calculated field at the exit of the guide then has to be transferred into the farfield as described below. The validity of this approximation is restricted to radiation with wavevektors which are less than the critical angle away from the optical (x-)axis. Therefore the approximation is well suited for x-ray waveguides which work at incidence angles smaller than $\alpha_{c}$ anyway. 


\subsection{The farfield distribution}

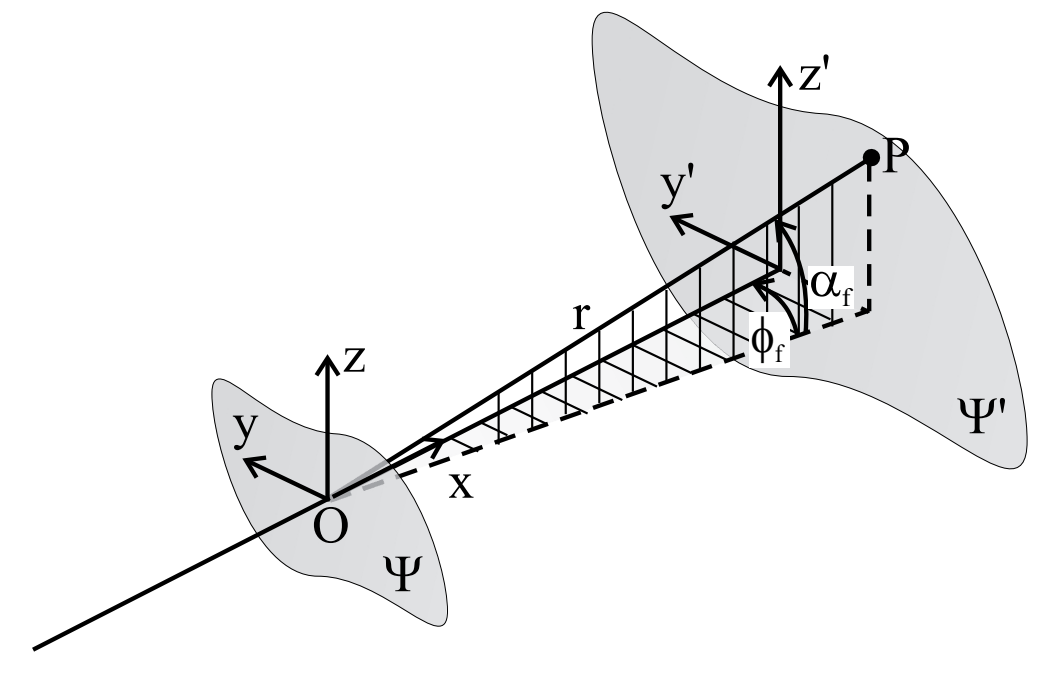

Figure 3.13: Variables and angles used for the calculation of the farfield pattern of a waveguide.

So far, concerning the field and intensity distribution, we have restricted the analysis to internal parameters. Due to the waveguide dimensions which are some ten nanometers in both directions only, these are not directly measurable. In order to find a connection to experimentally measurable variables we now analyze the pattern which is generated at a distance $D$ behind the waveguide exit where we can measure the intensity distribution with an adequate detector. This intensity distribution is called the farfield pattern of the waveguide. Since the detector distance is large compared to the waveguide dimensions, the intensity distribution in the farfield can be calculated according to the so called Fraunhofer approximation (Born and Wolf 1980). The two dimensional diffraction integral is given by:

$$
\Psi^{\prime}(l, m)=\int_{-\infty}^{+\infty} \int_{-\infty}^{+\infty} \Psi(y, z) e^{i k_{0}(l y+m z)} \mathrm{d} y \mathrm{~d} z
$$

for any field distribution $\Psi(y, z)$, where

$$
l=\frac{z^{\prime}}{r} \quad \text { and } \quad m=\frac{y^{\prime}}{r}
$$

and $r=\overline{O P}$ as depicted in Fig. 3.13. The intensity in the observation plane (detector screen) is therefore given by

$$
\mathcal{I}\left(y^{\prime}, z^{\prime}\right)=\left|\Psi^{\prime}\left(y^{\prime}, z^{\prime}\right)\right|^{2}
$$


which we can rewrite for the planar case using the approximation $\sin \alpha \simeq \alpha$ to

$$
\mathcal{I}^{q}\left(\alpha_{f}\right)=\mathcal{I}_{0}\left|\int_{-\infty}^{+\infty} \Psi^{q}(z) e^{i k_{0} \alpha_{f} z} \mathrm{~d} z\right|^{2}
$$

with q denoting the mode number. Similarly, for the two dimensional case we find

$$
\mathcal{I}^{p q}\left(\phi, \alpha_{f}\right)=\mathcal{I}_{0}\left|\int_{-\infty}^{+\infty} \Psi^{p q}(y, z) e^{i k_{0}\left(\phi_{f} y+\alpha_{f} z\right)} \mathrm{d} y \mathrm{~d} z\right|^{2} .
$$

These two equations however are valid for illumination of a single mode only as in the resonant beam coupling scheme, see section 3.3.1. Illumination of more than one mode $p q$ must be taken into account in the front coupling scheme, see section 3.3.2. Generalizing the described concept to two dimensional beam compression, the farfield distribution is then calculated by replacing $\Psi^{p q}$ by the sum of all excited modes. Therefore, $\Psi^{p q}$ in eq. 3.49 has to be replaced by the superposition

$$
\Psi(x, y, z)=\sum_{p q} c_{p q} \Psi^{p q}(y, z) e^{-i \beta_{p q} x} .
$$

The scaling factor $c_{p q}$ is calculated by the overlap integral of the field distribution of the respective mode and the incident field distribution $E_{i}(y, z)$ :

$$
c_{p q}=\frac{1}{\left\|\Psi^{p q}\right\|^{2}} \int E_{i}(y, z) \Psi^{p q}(y, z) d y d z
$$

In Fig. 3.14 the farfield distribution of a rectangular $50 \times 50 \mathrm{~nm}^{2}$ waveguide with refractive indices $n_{1}=1-0.5 \cdot 10^{-5}$ and $n_{2}=1-1.0 \cdot 10^{-5}$ at an x-ray wavelength $\lambda=1 \AA$ is shown neglecting absorption for the first six modes separately, while in Fig. 3.15 the farfield distribution of the device is shown for the case of front coupling a plane wave. According to eq. 3.51 only the $\Psi^{00}, \Psi^{20}, \Psi^{02}$ and the $\Psi^{22}$ modes are excited, while $c_{p q}$ is zero for all other modes which could possibly be excited. 

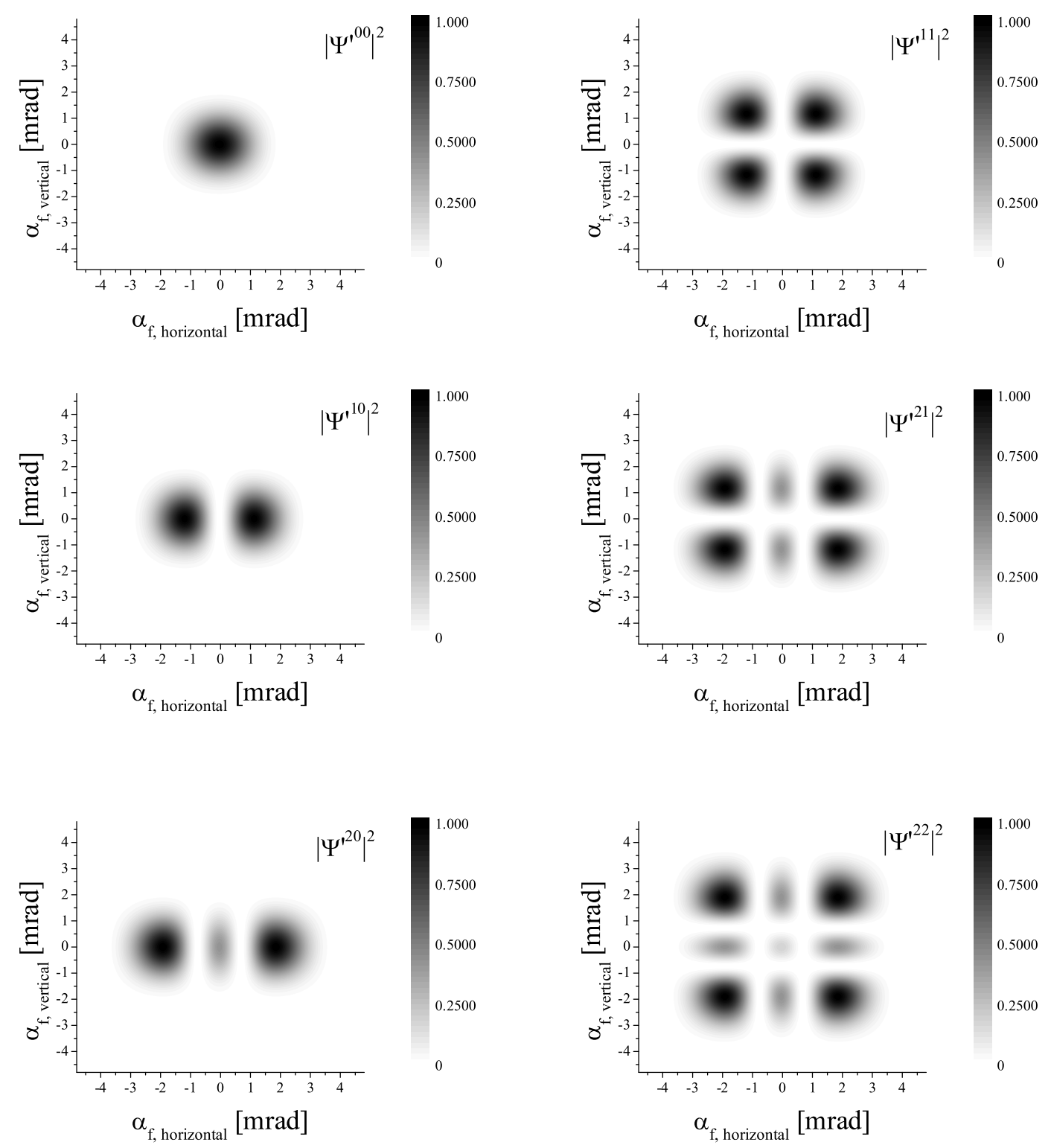

Figure 3.14: Normalized farfield intensity distribution $\left|\Psi^{p q}\right|^{2}$ of the $\Psi^{00}$ to $\Psi^{22}$ mode calculated according to eq. 3.49. The waveguide parameters are the same as in Fig. 3.7 where the corresponding internal intensity distribution is shown. The $x$-ray energy in this simulation was set to $12.4 \mathrm{keV}$ corresponding to an $x$-ray wavelength of $1 \AA$ 

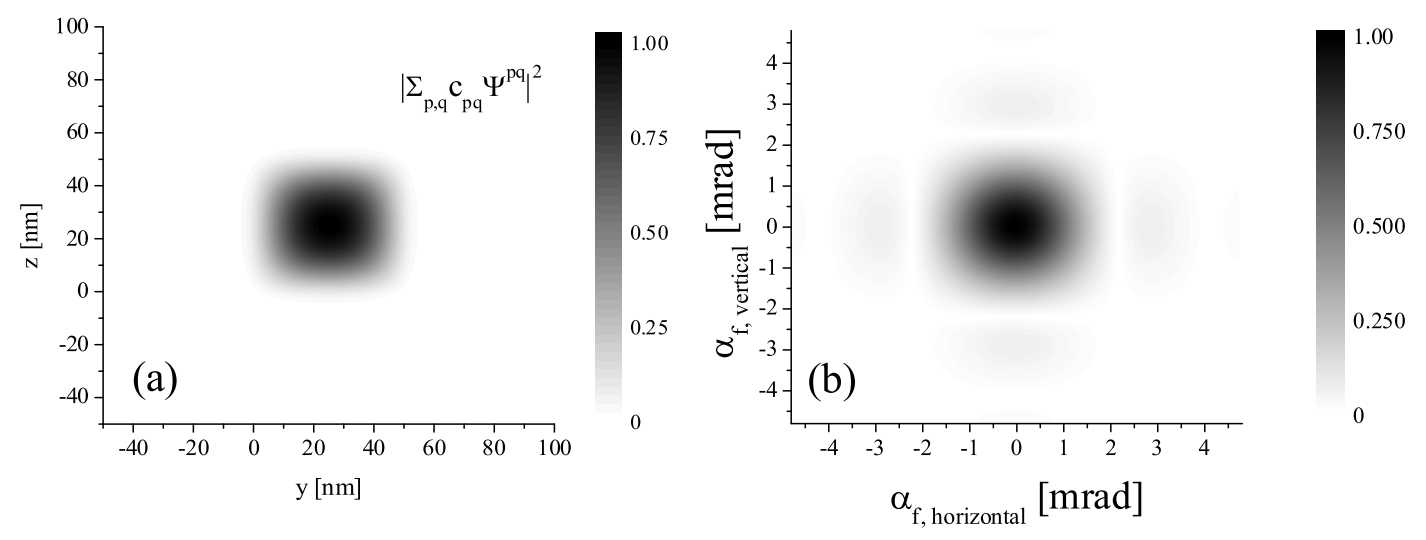

Figure 3.15: (a) Normalized intensity distribution due to mode mixing of the $\Psi^{00}, \Psi^{20}$, $\Psi^{02}$ and $\Psi^{22}$ mode in the waveguide $(x=0)$ described in Fig. 3.7 and (b) the corresponding farfield. 


\subsubsection{Farfield of a waveguide grating}

As we will see in the experimental section (chapter 6), the analysis of a single 2D waveguide requires high flux of the incident beam. In order to release this requirement a grating made up of $n$ individual waveguides can be studied (Seeger. 2004; Ollinger et al. 2005). For simplicity we will assume that the exiting beam profile of each individual guide is Gaussian with a width $w$. The presence of many $(n)$ of these is taken into account by convoluting this profile with Dirac $\delta$-function at positions $m d, m \epsilon[1, n]$, where $d$ is the distance between the individual guides. Changing the incidence angle $\alpha_{i}$ of the incident beam leads to a phase shift between the single channels corresponding to their lateral position. Finally we introduce an additional factor $N$ to model the lateral coherence length $N d$ of the incident beam. With these few assumptions we find the electric field $E\left(y, \alpha_{i}\right)$ at the waveguide exit:

$$
E\left(y, \alpha_{i}\right)=A\left(\alpha_{i}\right) e^{-2 x^{2} / w} \cdot \Sigma \delta(x-m d) e^{-i m d k_{i, y}} \cdot e^{-2 m^{2} / N^{2}},
$$

where $A$ scales the primary beam intensity, the efficiency of the waveguide grating and the angular acceptance of the individual waveguides. The farfield intensity distribution in the Fraunhofer approximation is then obtained by the relation

$$
\begin{aligned}
I\left(\alpha_{f}, \alpha_{i}\right) & =\left|\int E\left(x, \alpha_{i}\right) e^{\frac{i x 2 \pi \sin \alpha_{i}}{\lambda}} d x\right|^{2} \\
& =A^{2}\left(\alpha_{i}\right)\left|\frac{w}{2} e^{-\frac{1}{8} w^{2}\left(\frac{2 \pi}{\lambda} \sin \left(2 \theta-\alpha_{i}\right)\right)^{2}}\right|^{2}\left|\sum_{m} e^{i d m\left(\frac{2 \pi}{\lambda} \sin (2 \theta)\right)} e^{-\frac{2 m^{2}}{N^{2}}}\right|^{2}
\end{aligned}
$$

where $\alpha_{i}+\alpha_{f}$ was replaced by $2 \theta$. We see that the farfield consists of the well known grating peaks at fixed angular positions $2 \theta$. The farfield of a single channel defines an envelope to the farfield distribution of the grating. 


\section{Chapter 4}

\section{Fabrication of X-ray nanostructures}

In order to explore the use of various nanostructures for $x$-ray waveguide experiments, a variety of different systems and preparation techniques was applied. $1 D$ waveguides in resonant beam coupling $(R B C)$ geometry - prepared by spin coating and electron beam evaporation or by magnetron sputtering - were used for experiments with white synchrotron radiation. For the fabrication of two dimensionally confining waveguides, several fabrication techniques were investigated to obtain devices with high enough aspect ratios and small core sizes: Focused ion beam, templating from silk fibers, and electron beam lithography. Although not all of these devices turned out to be suitable for x-ray waveguiding, a description of all preparation methods is given in the following chapter.

\section{Substrate treatment}

For all preparation techniques except the focussed ion beam silicon wafers were used as substrates. A description of their pretreatment is therefore given here at the beginning of this chapter. The silicon substrates used for the waveguide fabrication are all cleaned from any contaminations before they are further processed. This is achieved by applying them to three different acid bathes and rinsing them in ultrapure water (Milli-Q, Millipore; Bedford, Mass.) in-between each step. The composition of the three bathes is given in the table below. 


\begin{tabular}{|c|c|c|c|c|c|}
\hline & acid & concentration & amount & temperature & duration \\
\hline $\begin{array}{l}1^{\text {st }} \text { bath } \\
(\mathrm{RCA} 1)\end{array}$ & $\begin{array}{c}\text { ammonia solution } \\
\text { hydrogen peroxide } \\
\mathrm{H}_{2} \mathrm{O}\end{array}$ & $\begin{array}{l}32 \% \\
30 \%\end{array}$ & $\begin{array}{l}1 \text { part } \\
1 \text { part } \\
5 \text { parts }\end{array}$ & boiling & $10 \mathrm{~min}$ \\
\hline $\begin{array}{c}2^{\text {nd }} \text { bath } \\
\text { (RCA2) }\end{array}$ & $\begin{array}{c}\text { Hydrochloric acid } \\
\text { hydrogen peroxide } \\
\mathrm{H}_{2} \mathrm{O}\end{array}$ & $\begin{array}{l}37 \% \\
30 \%\end{array}$ & $\begin{array}{l}1 \text { part } \\
1 \text { part } \\
5 \text { parts }\end{array}$ & boiling & $10 \mathrm{~min}$ \\
\hline $3^{r d}$ bath & hydrofluoric acid & $40 \%$ & 1 part & $\sim 20^{\circ} \mathrm{C}$ & $10 \mathrm{sec}$ \\
\hline
\end{tabular}

Table 4.1: To remove all organic ( $1^{\text {st }}$ bath, RCA1) and inorganic ( $2^{\text {nd }}$ bath, RCA2) contaminations, the silicon wafers which are used during the preparation are cleaned in different acidic bathes. RCA stands for Radio Corporation of America. The method was developed by (Kern 1993) at the RCA Sarnoff Research Center. The $3^{\text {rd }}$ bath takes away the native oxide layer.

\subsection{Thin films}

The preparation of both kinds of waveguides, $1 D$ and $2 D$, requires $n m$ sized layer systems. Their preparation was done either by spincoating, electron beam evaporation, magnetron sputtering or a combination of these techniques. On the next few pages they are briefly described.

\subsubsection{Spincoating}

Spincoating is an extremely successful technique for the preparation of thin films. It is relatively easy to handle and of comparatively low cost. The main restriction however is, that it only works with dissolved materials.

A sketch of the technique is shown in Fig. 4.1. A substrate, e.g. a quartz glass or a silicon wafer, is mounted on the sample holder of the commercially available spin coater. The substrate is usually held there by applying a vacuum between the holder and the sample. As mentioned before, the material from which the film is supposed to be made of (e.g. a polymer) has to be dissolved in a solvent. The concentration of this parent solution is one out of two parameters that determine the layer thickness in the end. A small amount of the solution, typically a few $\mu$, is pipetted onto the substrate. The sample holder is then accelerated to a prese- 


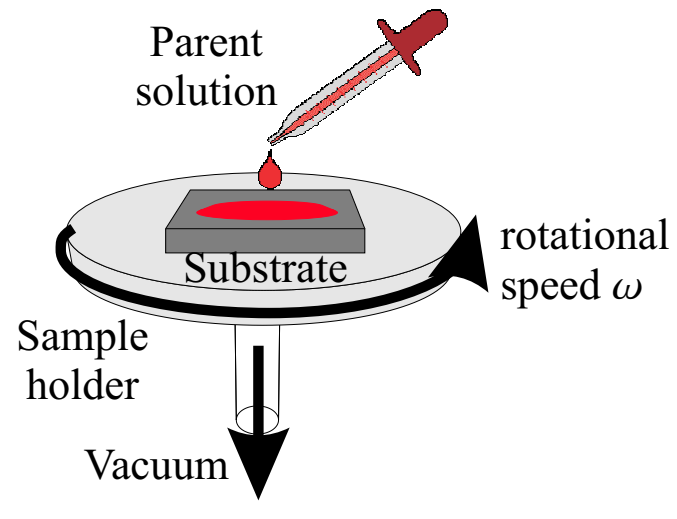

(a)

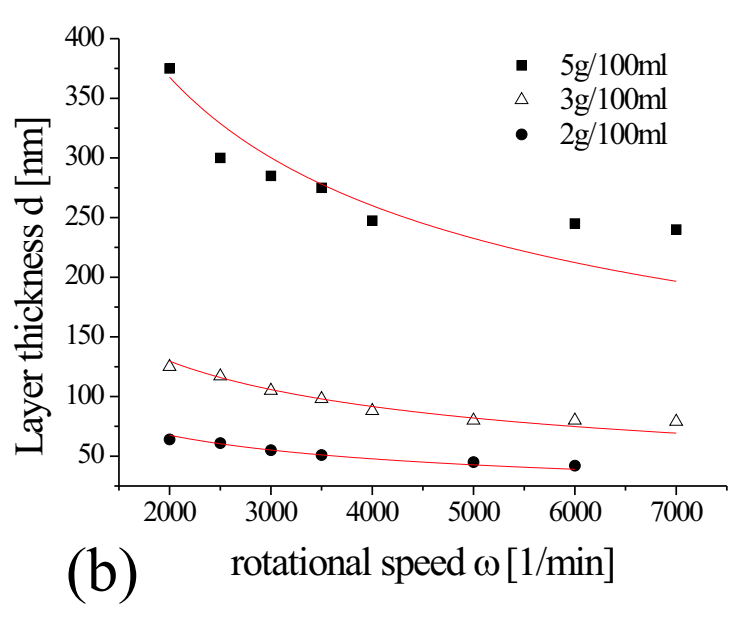

(b) rotational speed $\omega[1 / \mathrm{min}]$

Figure 4.1: (a) Sketch of spin coating system. With this technique, films of a few ten $\mathrm{nm}$ thickness can be prepared. (b) The layer thickness is proportional to the conceantration of the parent solution and inversly proportional to $\omega^{2}$. The lines are a fit to the measured data yielding $d[n m]=686 \cdot c^{2} / \omega$ (Seeger. 2004).

lected rotational speed $\omega$ of some thousand revolutions per minute (rpm), which is the second parameter determining the layer thickness $d$. During the rotation, the surface tension of the solution and the centrifugal force find their equilibrium condition resulting in a homogenous layer. The layer thickness $d$ is proportional to the square of the concentration of the polymer in its solvent and inversely proportional to the square of the rotational speed $d \propto c^{2} / \omega$ (Extrand. 1994). Residual solvent is removed by subsequent heating of the film in an oven or on a hot plate, which also serves for annealing of the film. For the present work the polymer PMMA (polymethyl methacrylate, Elvacite 2041, Leybold\&Voss\&Co) was spincoated, dissolved in either Chlorbenzene or in 2 methoxy ethyl acetate. The spincoating technique allows for the preparation of PMMA layer thicknesses from $10 \mathrm{~nm}$ up to more than $100 \mathrm{~nm}$ with an accuracy $\leq 2 \mathrm{~nm}$ as determined in x-ray reflectivity measurements. The specific preparation parameters are given in the respective preparation sections of this thesis. 


\subsubsection{Electron beam evaporation}

For the preparation of thin films with insoluble materials such as silicon or chrome, an electron beam evaporation system was used (UNIVEX 450, Leybold). The technique is relatively simple and inexpensive and is thus available for many laboratories. The design is shown in principle in Fig. 4.2. The target material is kept inside

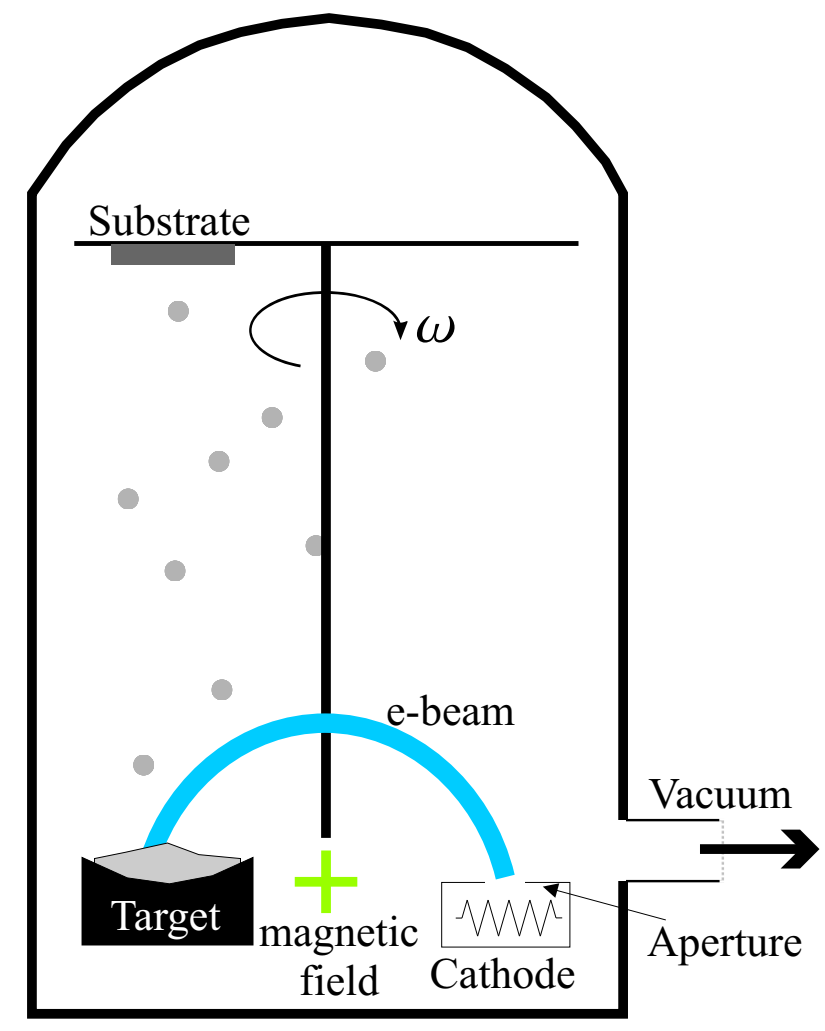

Figure 4.2: Sketch of the e-beam evaporation System at the x-ray physics institute in Göttingen.

a crucible in the evaporation chamber of this system. It is heated by an electron beam to evaporate the respective material. The evaporation flux $d N_{e} / A_{e} d t$ follows the Hertz-Knudsen-Langmuir equation (Langmuir 1913, 1916):

$$
\frac{d N_{e}}{A_{e} d t}=\alpha_{\nu}\left(2 \pi m k_{B} T\right)^{-\frac{1}{2}} P
$$

where $P$ is the vapor pressure of the target at temperature $T, a_{\nu}$ is the sticking coefficient for vapor molecules onto the surface, $m$ is the atomic mass and $k_{B}$ 
is the Boltzman constant. In our setup, the vertical distance between the target crucible and the wafers is $\sim 30 \mathrm{~cm}$. During the evaporation the sample rotates at a rotational speed $\omega \simeq 30 \mathrm{rpm}$. Its distance to the center of rotation is $\simeq 170 \mathrm{~mm}$. This setup guarantees a homogenous cover of the sample. The deposition rates are in the range of four to ten $\AA$ per second. Electron beam evaporation was used for the deposition of Si and Cr for total layer thicknesses ranging from $6 \mathrm{~nm}$ up to 300 nm.

\subsubsection{Magnetron sputtering}

For some materials such as carbon for example, magnetron sputtering is the better choice for thin film production, at least if this technique is available. The $\mathrm{Ni} / \mathrm{C} / \mathrm{Ni}$ waveguides which were used for some of the white beam experiments were prepared at the sputtering facilities of the ILL ${ }^{1}$ by Franz Pfeiffer (Pfeiffer. 1999, 2002). A sketch of a magnetron sputtering setup is shown in Fig. 4.3.

A magnetron consists of a plate of the coating material (target). Behind the target, magnets are arranged which create a magnetic trap for charged particles, such as argon ions, in front of the target. Putting the target at a negative voltage attracts argon ions to the target. When they hit the surface, two important processes take place: i) Atoms are knocked out of the target surface by the ions - this is sputtering. These sputtered atoms are not charged, so they go straight out of the magnetic trap. It is these atoms which hit the substrate to form the coating. ii) The surface also releases electrons. These are held in the magnetic trap, where their energy is used to produce more argon ions. This means that the ions which are attracted to the surface are replenished all the time, so that the magnetron can operate continually. The combination of pumping down to the high vacuum region and the continuous throughput of process gas ensures that coating conditions are both clean and reproducible.

\footnotetext{
${ }^{1}$ Institut Laue-Langevin, Grenoble, France
} 


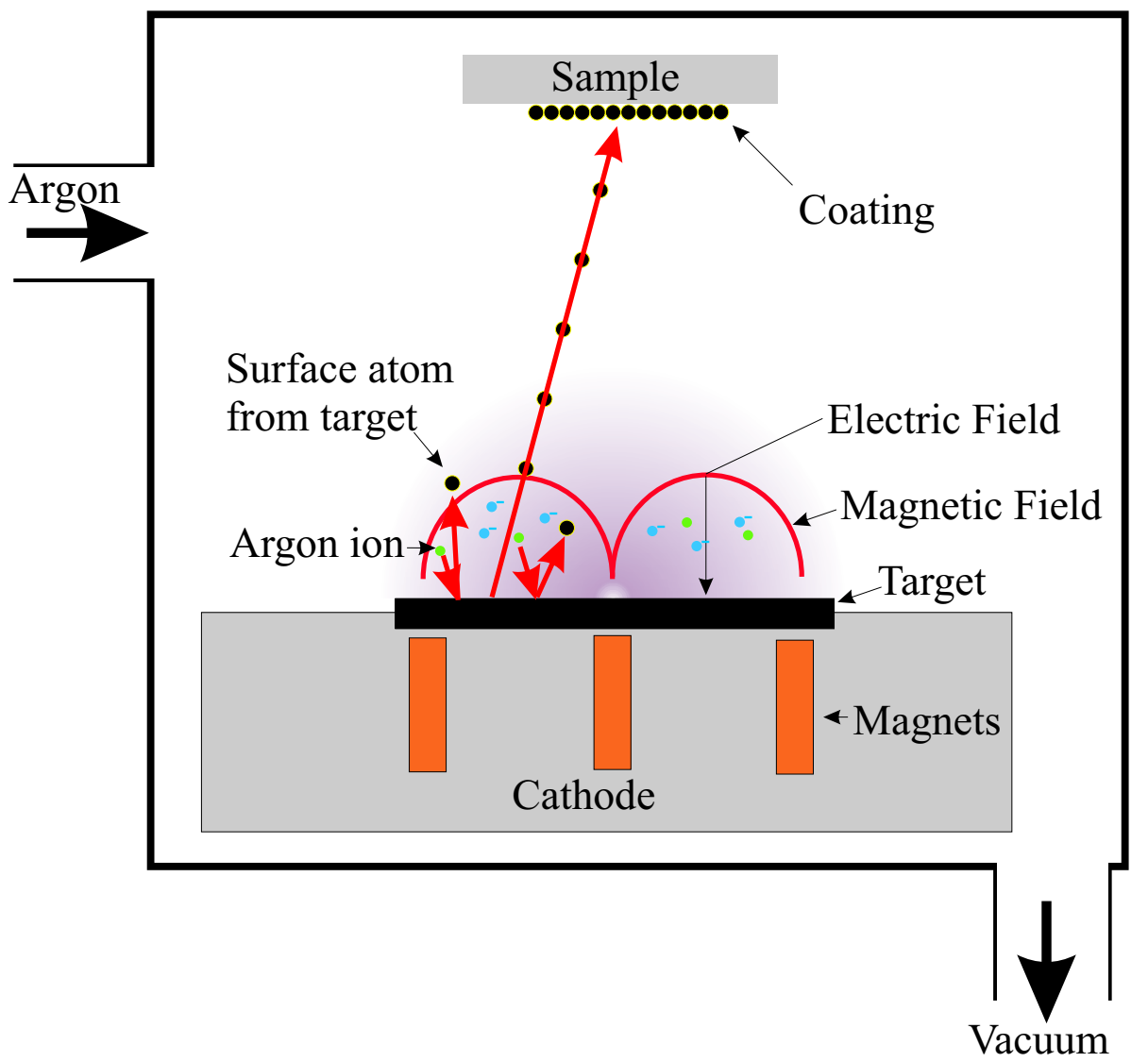

Figure 4.3: Typical setup of a magnetron sputtering facility. 


\subsection{DWG-fabrication using Focused ion beam}

In order to achieve sub micron $2 \mathrm{D}$ front coupling waveguides, one can make use of those established techniques which work on the same structure sizes as the desired guide is supposed to hold. One of these techniques is the Focussed Ion Beam (FIB) which is widely used for sputtering, deposition and, most important to us, for milling. The idea is to use the focussed ion beam to drill small holes into thin metal foils. In order to ease the break-through of the accelerated ions through the foil, these have to be kept as thin as possible. Therefore, high $\mathrm{Z}$ elements must be used in order to achieve the required absorption of the primary x-ray beam. The thickness of the foil has to be adopted to the energy of the respective experiment. A quick calculation gives the minimum thickness at an x-ray energy of e.g. $10 \mathrm{keV}$. A $20 \mu \mathrm{m}$ thick Ta-foil for example yields a transmission of the beam of $3.7 \times 10^{-4}$. This would be sufficient to detect the guided beam. On the other hand, a diameter of 300 $\mathrm{nm}$ or less is required in order to achieve waveguiding. Consequently, the minimum aspect ratio (length of the hole over its width) needed is in the order of 50 to $60^{2}$. In
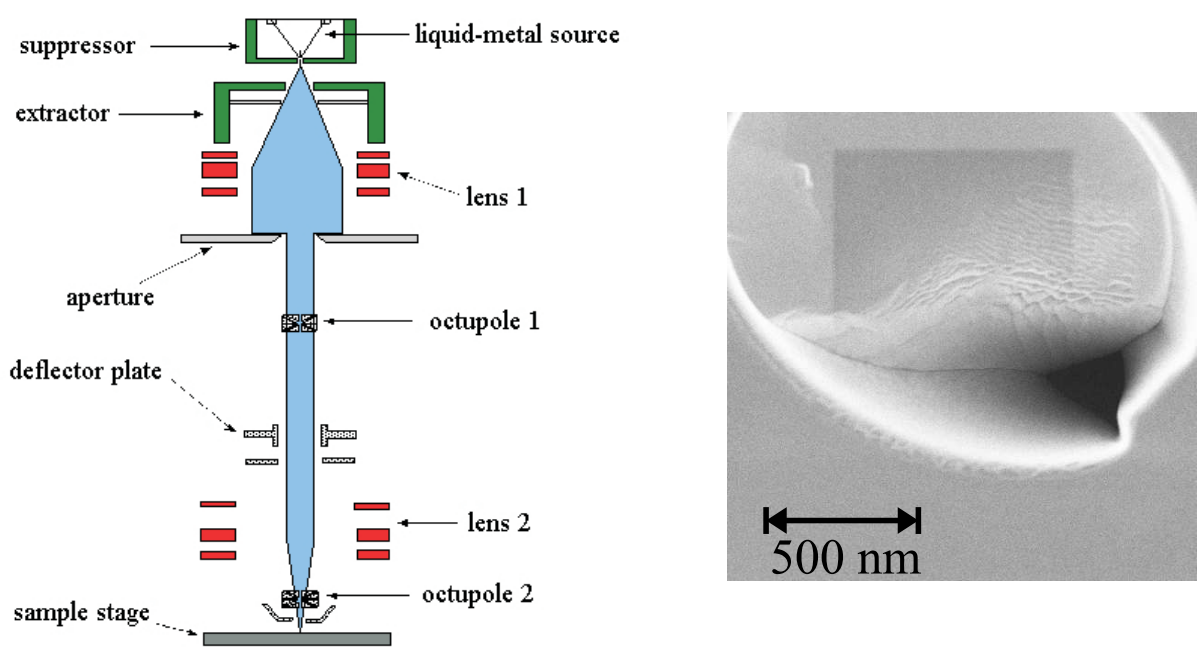

Figure 4.4: Sketch of a focussed ion beam milling machine (left) used to drill holes into thin (20 $\mu \mathrm{m})$ Tantal foils (right).

cooperation with Dr. L. Bischoff from Forschungszentrum Rossendorf (FZR), Dr. H.

\footnotetext{
${ }^{2}$ Please note that for lower aspect ratios the formalism does not change, but the waveguide then starts to behave more and more like a pinhole.
} 
Wendrock from Institut für Festkörper- und Werkstofforschung (IFW) Dresden and Dr. C. Trautmann from Gesellschaft für Schwerionenforschung (GSI), Darmstadt, both, Ta-foils for hard x-rays and Kapton-foils for the soft x-ray regime of different thickness $d$ were treated in a $\mathrm{Ga}^{+}$ion beam to produce smallest possible holes. To fabricate Kapton nano pores, a track-etching technique was employed, based on irradiation of a polymer film with heavy ions of total kinetic energy in the GeV range, and subsequent chemical etching of the latent ion tracks (Mara et al. 2004). The number of pores created in this way is equal to the number of ions allowed to penetrate the foil (in our case between 0.1 and 1 pores $/ \mu \mathrm{m}^{2}$ (averaged). The size and shape of the pore produced can be tailored with a precision of nanometers by controlling the type of etchant, temperature, and duration of etching. $12 \mu \mathrm{m}$ thick Kapton membranes with pore diameters of 50 to $300 \mathrm{~nm}$ were prepared at the GSI. However, in the soft x-ray regime, the Kapton foils were found to be severely effected by the energy deposited by the $500 \mathrm{eV}$ beam. Other Materials might work better, but the aim of the present work was to go for a hard x-ray nanobeam and no further experiments were performed in the soft x-ray regime. Hard x-rays in turn require higher $\mathrm{Z}$ elements where ion track etching is not established to our knowledge and milling is the best way to treat these membranes. Yet, milling Ta foils to a critical depth leads to enhanced redeposition of the milled material in the hole, which prevents the ion beam from milling deeper. A variation of the beam current (70 pA - $600 \mathrm{pA})$ could not overcome this limitation. The size of the successfully drilled holes in a $20 \mu \mathrm{m}$ Ta foil therefore was larger than desired and, in addition, their shape became uncontrollable, at least at the entrance and at the exit of the guide. Experiments showed that these devices can guide an x-ray beam, but the restrictions prevent a useful and controlled application of these devices. Fig. 4.4 shows both, the principal setup of a focussed ion beam, which is similar to an electron beam lithograph, and a small hole which was drilled at $600 \mathrm{pA}$ for 83 minutes. The astigmatism of the hole is probably caused by an asymmetric beam profile in high current mode. 


\subsection{Templating from spider silk fibers}

Nature offers a variety of thin fibers which can be used to fabricate the extremely fine structures needed for two dimensionally confining hard $x$-ray waveguides. Using elastic materials such as e.g. spider silk offers the advantage, that these tend to be straight under tension. Secondly they are available in nearly any diameter (ranging from $\sim 10 \mathrm{~nm}$ (Stegodyphus pacificus) up to several microns (e.g. Nephila clavipes)) and in any desired length up to several meters which allows for high aspect ratios (length/diameter).

The basic idea is very simple: In a first step, the fibers have to be milked from that spider that produces the desired fiber diameters. After cleaning these fibers, they are coated with a higher $\mathrm{Z}$ element which will act as the cladding of the waveguide. Finally, the devices have to be cut to the desired length and tested in the experiment.

Spiders of the genus Araneus diadematus (garden spider) are held at an air mois-

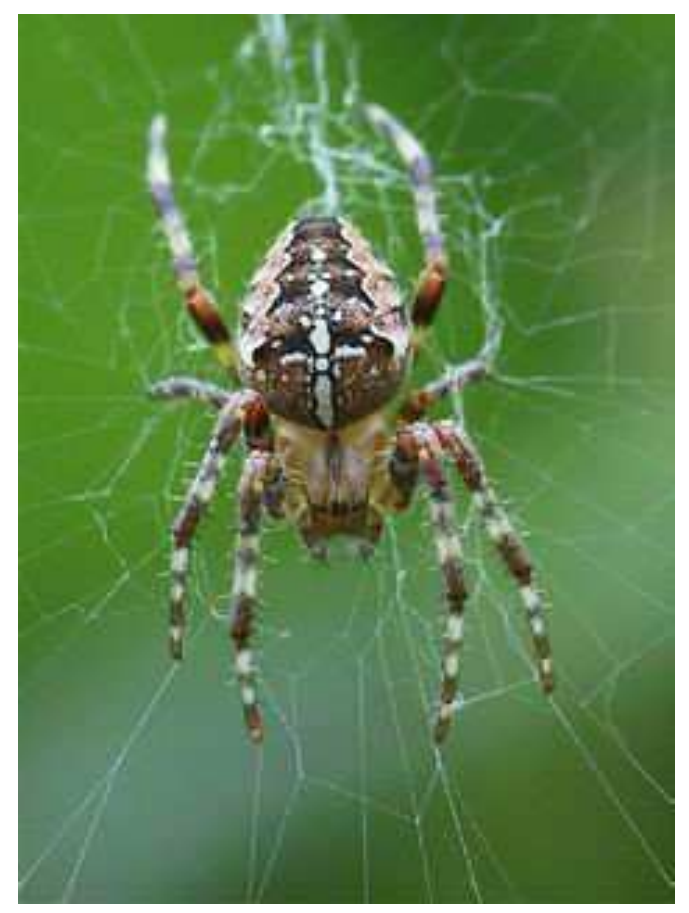

Figure 4.5: Araneus diadematus (garden spider; image from natur-lexicon.com) in the middle of her web. Its silk fiber could act as the core of an $x$-ray waveguide. 


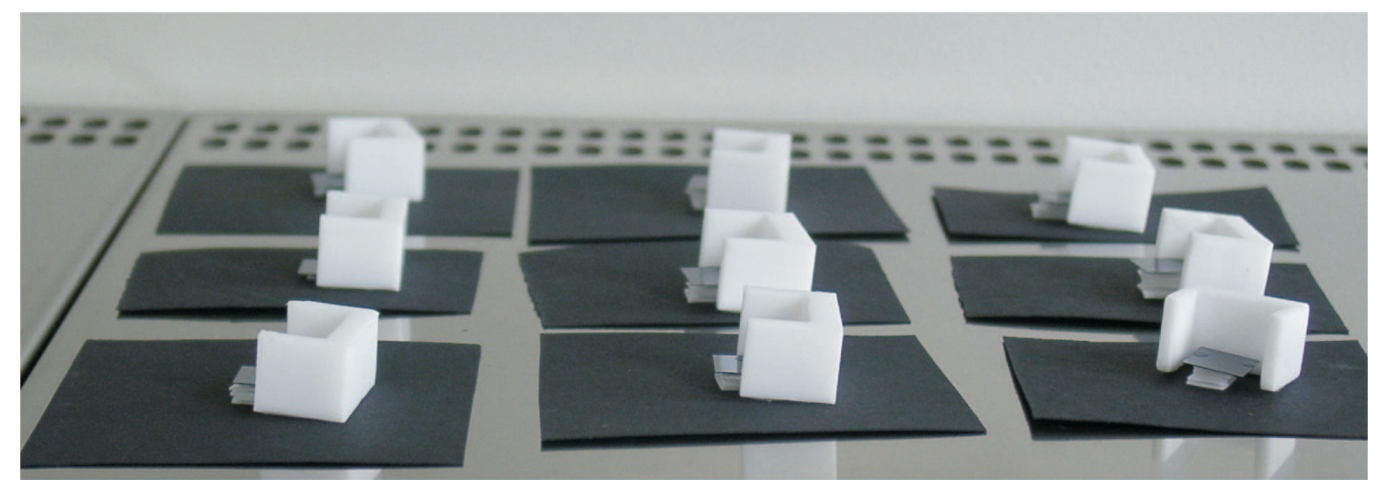

Figure 4.6: The harvested and cleaned silk from the garden spider is spanned across cleaned silicon wafers.

ture of $75 \%$ at $25^{\circ} \mathrm{C}$. In order to milk the silk, the spiders are cooled down in a standard refrigerator at $-5^{\circ} \mathrm{C}$ in order to immobilize them (garden spiders can stand temperatures down to $-20^{\circ} \mathrm{C}$ ) (Glisovic. 2004). After one hour, the spiders are taken out and hereafter attached leg by leg and bottom up to a black card board (without wounding them), since the silk producing glands are at the abdomen of the spider. Different glands produce different fibers, so only one of them has to be 'activated': the respective gland is smoothly stroked with a fine brush, which by reflex makes the spider produce the silk. For the first experiments we chose the dragline of the garden spider, which is roughly $1 \mu \mathrm{m}$ in diameter, depending also on the size of the spider. After coiling the silk onto a metal grid, the long fiber is cut into pieces of $\sim 2 \mathrm{~cm}$ and attached to a teflon-u with epoxy resin. The untreated silk is surrounded by a coverage of sericin, which protects the silk from desiccating and acts as adhesive. This sericin coating can be removed in a $\mathrm{KOH}$ bath, where the silk is kept at pH 10 for 30 minutes at $60^{\circ} \mathrm{C}$. After drying the fibers in a flowbox, they are spanned onto a cleaned silicon wafer. Therefore the teflon-u is turned upside down. Putting the side pieces of the $u$ to the left and right of a $10 \times$ $10 \mathrm{~mm}^{2}$ Si-wafer makes the silk span over the entire length of the wafer (see Fig. 4.6). Dragline from the garden spider can be spanned to up to $140 \%$ of its original length. This is used to make sure, that the strain of the silk automatically ties the fiber straight (see Fig. 4.7 a): Additional spacers are placed below the wafer so that the side pieces of the Teflon-u do not touch the ground any more, held only by the strain of the silk. Hereafter the silk is glued carefully onto the Si-wafers and kept in a flowbox over night.

In a next step another $300 \mathrm{~nm}$ of $\mathrm{Si}$ are evaporated atop the wafer and the silk. 
After evaporation, the wafers have to be cut to the desired length. This is the most sophisticated part in the preparation. The silk exhibits astonishing properties concerning its resistance towards outside influences: Breaking the wafers without further treatment of the silk leads to the fact that the extremely stable silk rips off huge parts of the evaporated silicon cladding due to its enormous tensile strength (see Fig. 4.7 e). Therefore, the samples were cooled down in liquid nitrogen (LN) before breaking in order to make the silk brittle. This attempt however is not recommendable. A short story that happened in our lab: One of the silk wafers was scored with a diamond cutter perpendicular to the silk direction on its backside before dipping it into LN for 2 minutes. Immediately after taking the wafer out again, it was cleaved alongside the chink with a time delay of about 3 seconds which guaranteed that there was no time for thermal equilibration to room temperature. While the wafer broke apart, the silk was not affected at all and held the two pieces together. The two silicon pieces were connected only by the spider silk. This double piece was held into the LN again, to test the behaviour of the silk. Upon swaying it fiercely, it was found that the silk was not stiff at all, but still tearproof, elastic and flexible. This behaviour at $-196^{\circ} \mathrm{C}$ was as astonishing as disadvantageous for the waveguide fabrication.

A more successful attempt to break the wafers was to vaporize the silk at high temperatures. For this purpose the samples were placed in an oven at high temperatures $\left(500-1000^{\circ} \mathrm{C}\right)$ until the silk was destroyed. While LN temperatures did not effect the cladding, high temperatures were found to do so. Some examples are shown in Fig. 4.7. The least rejects were produced at $620^{\circ} \mathrm{C}$ and a baking time of ten to twelve minutes. If not damaged, these samples were cut to the length required for experimental examination. 

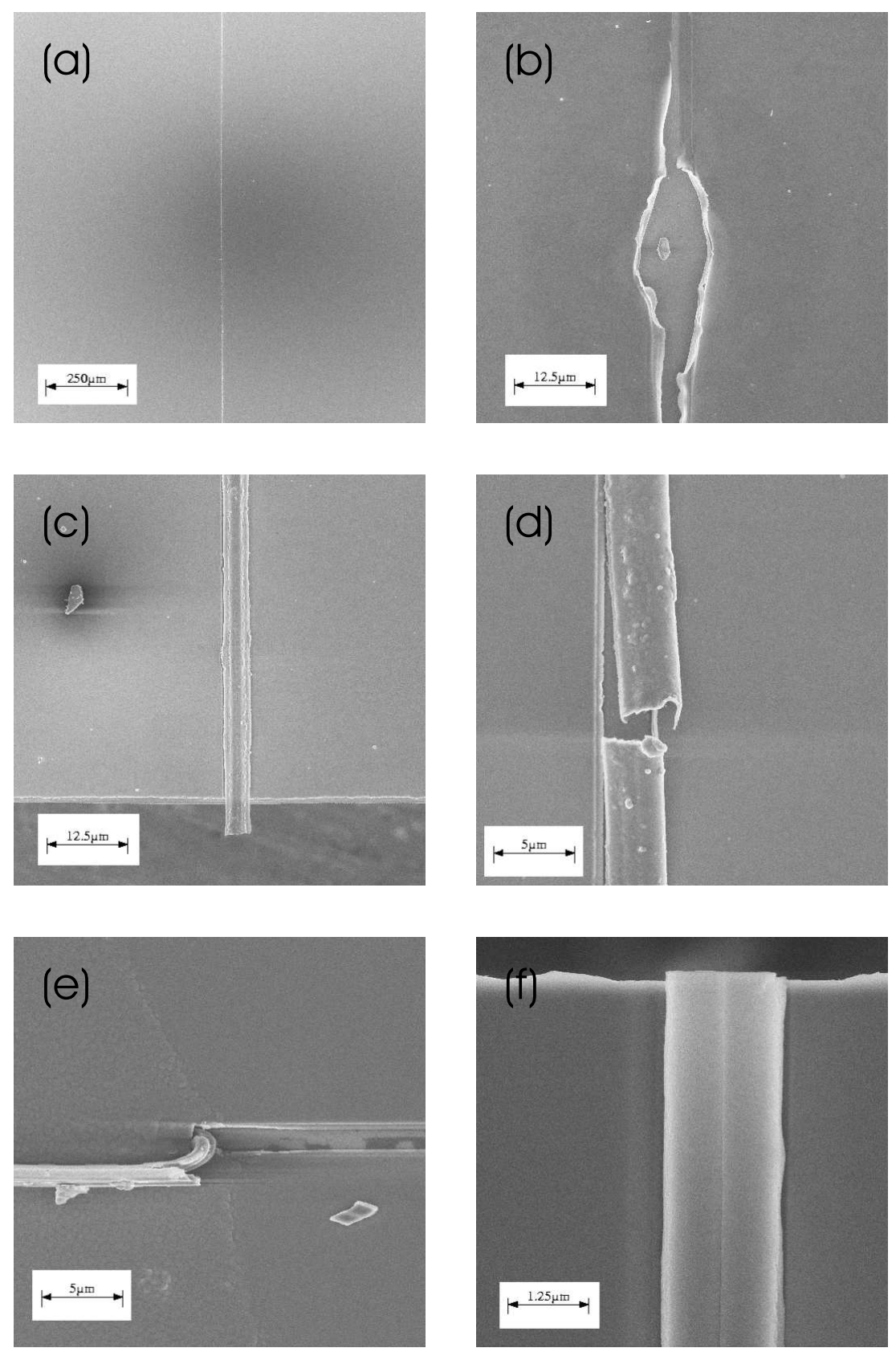

Figure 4.7: Silk from the garden spider on silicon wafers after evaporation of another $300 \mathrm{~nm}$ Si and after vaporizing the silk at 620 $\mathrm{C}$ seen by the SEM (Acceleration Voltage $5 \mathrm{keV}, 17 \mu \mathrm{m}$ aperture). (a) The silk is spanned dead straight across the silicon wafer. (b) The baking procedure often causes severe damage to the guide. (c) This channel was not damaged during the baking process. The cladding stands above the wafer after breaking the guide to the desired length. (d) Very often the channel breaks and one side lifts off a little bit. (e) Without baking, the silk rips off the cladding during the breaking of the wafers. On the left side, where the channel break ends, the glue which attaches the silk to the wafer is seen below the cladding. (f) Neat entrance of a double fibre. This is not wanted, but it is a good test sample. $\quad-54$ - 


\subsection{Electron beam lithography}

Electron beam lithography $(E B L)$ is a specialized technique for creating the extremely fine patterns required e.g. by the modern electronics industry for integrated circuits. Derived from the early scanning electron microscopes, the technique in brief consists of scanning a beam of electrons across a surface covered with a resist film. The beam deposits energy in the resist film and increases (or decreases) its solubility in an adequate solvent. EBL offers highest resolution down to a few nm. Furthermore, there are only few restrictions to the patterns that can be written. Therefore, EBL is a promising technique for the fabrication of two dimensionally confining x-ray waveguides.

All patterning of the devices described in this section was performed using the Leica LION LV1 ${ }^{3}$ electron beam writer. Essentially it consists of a substrate holder with an interferometrically controlled xy-stage, high quality vacuum units, a vibration isolated electron optical column, a data processing system, an exposure control unit and control electronics with the operating software. The electrons are generated by heating a Shottky field emitter. They are accelerated by a high voltage $(8 \mathrm{keV})$ to reduce the effect of any disturbing external magnetic field and guided through an aperture of variable width $(120,60,30,17$ or $10 \mu \mathrm{m})$ to set the beam current and dimensions. To reduce the Coulomb interactions between the electrons, they are guided down the column by electrostatic lenses without being focused, i.e. without any crossovers of the electron pathes which prevents from considerable energy spreading and thus also from beam spreading in the lenses further downstream. Before the beam hits the sample, the electrons are slowed down to their final energy between 1 and $20 \mathrm{keV}$. Hereafter the beam is focused to the sample with a minimum diameter of 2 to $5 \mathrm{~nm}$, depending on the respective aperture chosen. Nevertheless, the patterns that can be generated are limited not only by the beam size, but also by what is known as the proximity effect and the stiching error.

Stitching error: Most EBL-systems use the deflection of the electron beam to pattern the sample. Since there is a maximum deflection angle of the beam, the area, which can be patterned without moving the sample is limited (e.g. 180·180 $\mu \mathrm{m}^{2}$ working field for the LION). In order to write larger fields, the whole pattern is divided into small areas, which are processed consecutively, while the sample

\footnotetext{
${ }^{3}$ Lithography System for Integrated Optics and Nanometric Structures - Low Voltage $1 \mathrm{kV}$, Leica Lithographiesysteme GmbH, Jena
} 
is moved into a new position after the completion of each of these areas. With this technique, a slight misalignment between the desired and the actual sample position can not be avoided, from what follows the so called stiching error. This problem can be circumvented in the Continuous Path Control mode (CPC) where the sample moves continuously on the interferometrically controlled table, using the deflection of the e-beam only for corrections between the desired and the given sample position.

Proximity effect: As the electron beam penetrates the resist layer, it undergoes a complicated scattering process. Hitting a molecule, a high-energy electron scatters into an arbitrary direction and creates a flow of secondary electrons. Since these do not have enough energy to travel very far, they only contribute to the effective spreading of the electron path along with the forward-scattering of the primary electrons. However, some of the primary electrons can penetrate the resist and scatter back from the surface of the underlying substrate. This results in considerable spreading of the exposed area compared to the spot size of the exposing beam. The effect is called the proximity effect. The proximity effect is an intrinsic part of electron beam lithography and can never be completely eliminated. It can however be reduced, by choosing a lower acceleration voltage and thus minimizing the number of electrons that is backscattered from the substrates surface.

Before we can start with the description of the actual waveguide preparation, we need to specify the properties of the resist. There are two groups of resists used in EBL, as there are the so called positive resist, and the negative resist. An example of a positive resist is the polymer polymethyl methacrylate (PMMA): During the exposure the electrons break up the long-chain PMMA molecules into smaller molecules, which can be solved by a developer afterwards, i.e. those areas which were exposed are washed away during the development. The developer used in the present work was a (4:1) mixture of ethyleneglycolmonoethylether and ethyleneglycolmonobutylether (both from Merck-Schuchardt). The patterns were applied to a bath of these two chemicals at room temperature for 45 seconds and rinsed in isopropanol afterwards to stop the development. A negative resist behaves inversely: Upon e-beam exposure cross linking is induced. In the developer again only short molecules are washed away. Thus, in the case of the negative resist, the exposed areas of the resist remain on the substrate.

The fabrication of 2-dimensionally confining waveguides using PMMA as resist for EBL is described in the following sections. 


\subsubsection{V- and U-grooves}

For the preparation of V-shaped grooves in Si (see Fig. 4.9), a thin Cr layer (15 $\mathrm{nm}$ ) is sputtered onto a cleaned (100)-oriented (110-orientation for the U-groves) Si-wafer which exhibits an additional oxide layer of $15 \mathrm{~nm}$ thickness. Note that these wafers are not applied to hydrofluoric acid for cleaning. The PMMA parent solution (the polymer is bought dissolved in chlorbenzene at a concentration of $9 \%$ ) is further thinned by mixing it with additional chlorbenzene (1:6). Spincoating at

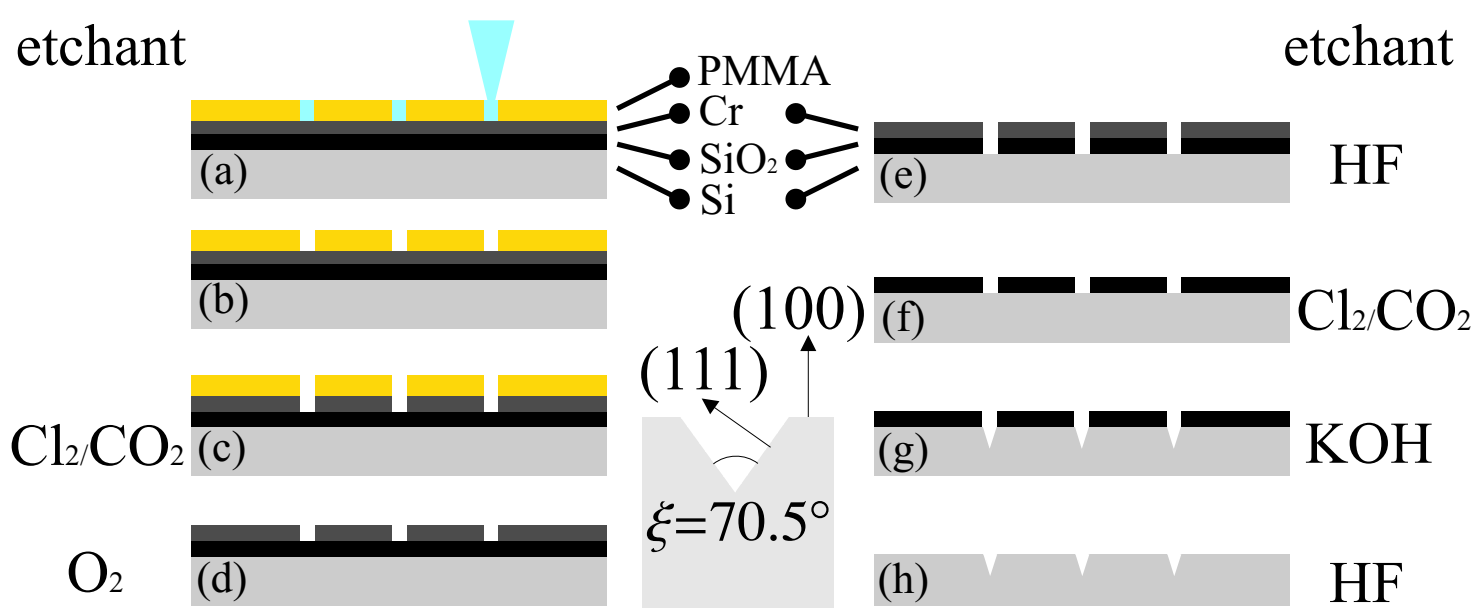

Figure 4.8: The preparation of V-grooves demands a layer system where each layer acts as etch mask for the next one. (a) The electron beam writes structures into the PMMA resist which (b) results in the desired pattern after development of the resist. (c) The underlying $\mathrm{Cr}$ layer is etched by plasma etching with $\mathrm{Cl}_{2} / \mathrm{CO}_{2}$. (d) Residual PMMA is removed by plasma etching with $\mathrm{O}_{2}$. (e) The pattern is next transferred to the $\mathrm{SiO}_{2}$ layer by wet etching with $\mathrm{HF}$ followed by $(f)$ another $\mathrm{Cl}_{2} / \mathrm{CO}_{2}$ etching to remove the $\mathrm{Cr}$ completely. (g) Anisotropic etching with KOH finally transfers the pattern into the Siwafer. (h) The $\mathrm{SiO}_{2}$ is removed with HF. (100)-oriented wafers form V-shaped grooves, while with the same method (110)-oriented wafers form rectangular grooves.

$3000 \mathrm{rpm}$ and subsequent baking at $170^{\circ}$ ( 1 minute on a hot plate) results in a $50 \mathrm{~nm}$ thin resist layer. In the electron beam writer long lines are written into the resist at a linedose of $0.25 \mathrm{nC} / \mathrm{cm}$ with a $17 \mu \mathrm{m}$ aperture. Next, the written pattern is developed as described on page 56. The pattern can now be transferred into the underlying Cr-layer by anisotropic plasma etching with $\mathrm{Cl}_{2} / \mathrm{CO}_{2}$ for $60 \mathrm{~s}$. 
The residual PMMA is removed by plasma etching with $\mathrm{O}_{2}$. Hereafter, the wafer is dipped into a $1 \%$ hydrofluoric acid (HF) solution at room temperature which transfers the pattern into the $\mathrm{SiO}_{2}$-layer within 2 minutes. The $\mathrm{Cr}$ which is not needed anymore can be removed by another $\mathrm{Cl}_{2} / \mathrm{CO}_{2}$ plasma etching step. Now the pattern can be etched into the $\mathrm{Si}$ in a 10wt\% Potassium Hydroxide (KOH)bath at $56^{\circ} \mathrm{C}$ which takes roughly $1 \mathrm{~min}$ for a $100 \mathrm{~nm}$ wide line, while wider grooves need suitably more time, as they will be deeper (see below). KOH etches Si anisotropically. The etch speed in (111)-direction is slowed down by roughly two orders of magnitude compared to all other directions (Yamamoto et al. 1988). For this reason, the grooves are $\mathrm{V}$-shaped with an opening angle $\xi$ of $70.5^{\circ}$ (U-shaped in the case of 110 -orientation). The residual $\mathrm{SiO}_{2}$ is removed in a final $\mathrm{HF}$ bath (2 min, as before). The width of the pattern is mainly determined by the lithography process. There is a slight broadening to the original line width due to the HF etching (step (e) in Fig. 4.8), since in this isotropic wet etching step an under etching of the above lying Cr-layer can not be avoided. This effect however can be kept small by minimizing the HF etching time. Since the width $w$ of the grooves is determined mainly by the lithography process, the depth $h$ of these grooves is limited to $h=w / \sqrt{2}^{4}$. At this point, I would like to thank Dr. C. David ${ }^{5}$, who provided all his expertise on nano structuring, for his help and for the convenient atmosphere during numerous fruitful discussions on this topic.

Etching the (V-shaped) grooves is half the work done. In order to make these devices waveguides, they have to be covered with Si. Two attempts were performed to achieve this goal after cutting the grooves and cleaning them again to remove all contaminations: (a) straightforward covering with another Si-wafer cut to the same length and holding them tight in a small bench vice and (b) waferbonding of the two pieces.

\subsubsection{Wafer bonding}

When two atomically smooth and clean surfaces are brought into intimate contact they adhere to each other via intermolecular forces such as van der Waals forces or hydrogen bonding, see e.g. (Tong and Gösele 1998). Since the technique is well established e.g. in semiconductor industries, Si-wafer with the required flatness are commercially available. Nevertheless it is very challenging to achieve the de-

\footnotetext{
${ }^{4}$ for U-groves the depth is limited by the etching time tabled in (Williams and Muller. 1996).

${ }^{5}$ Paul Scherrer Institut, Villigen, CH
} 
sired bonding, since any contaminations of the surfaces prevent successful bonding. Therefore, before bonding, both the structured and a blank Si wafer were cleaned as described on pages $43 f$ and put together immediately afterwards. Experimental examination of bonded U-groove gratings evidenced that the technique in principle is suitable for waveguide preparation (see chapter 6.3.2), but only the new clean room facilities which are currently built at the physics department will allow for a standardization of this process at the University of Göttingen.

\subsubsection{Polymer core waveguides}

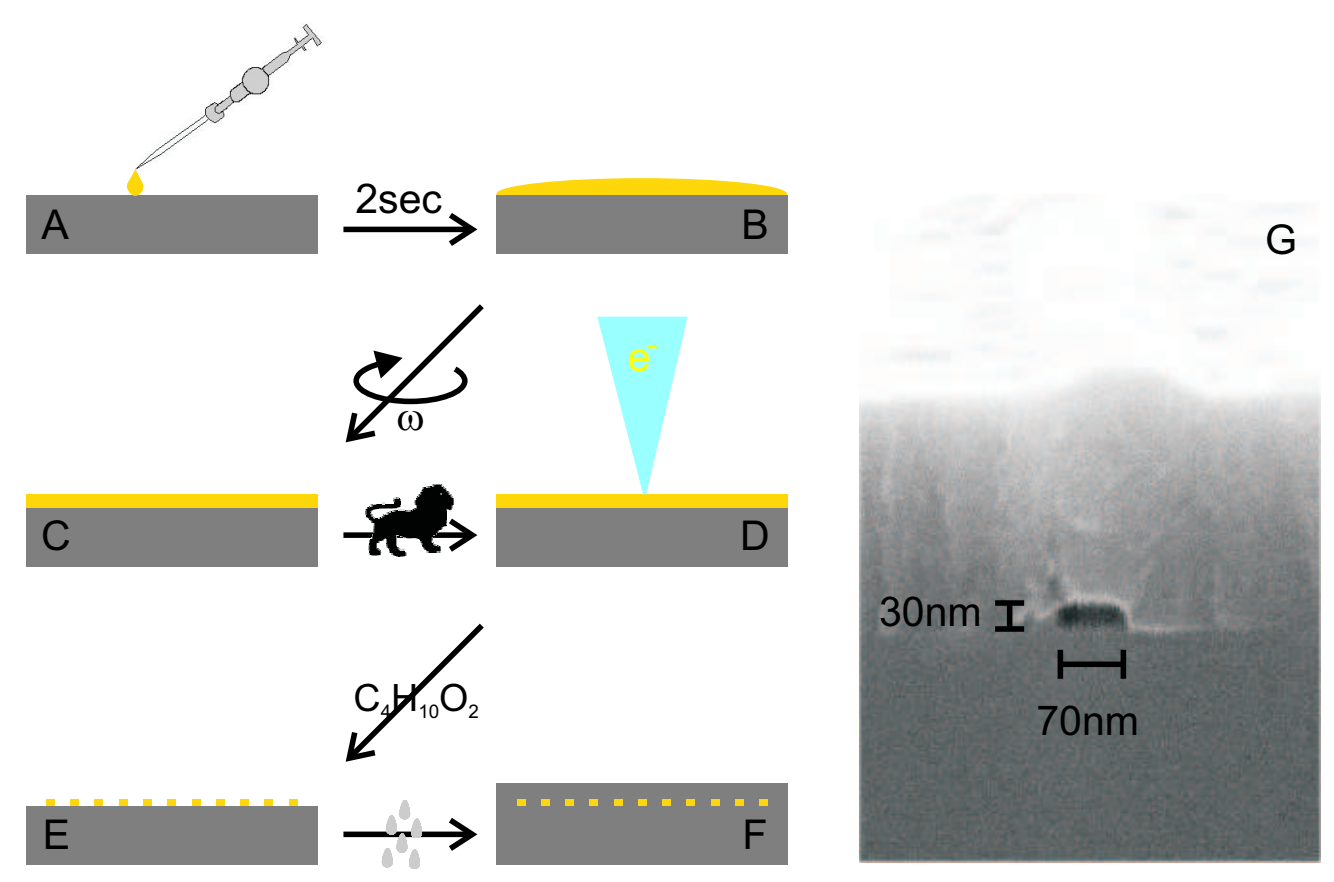

Figure 4.9: Preparation of polymer core waveguides: $A-C$ : $A$ drop of the dissolved polymer (PMMA) is pipetted onto the thoroughly cleaned Si-wafer wetting the substrate. Next, the wafer is spun at a rotational speed of 4000-5000 rpm yielding a thin layer of some ten $\mathrm{nm}$ thickness. D: The PMMA layer acts as a positive resist in e-beam lithography (LION LV1, Leica). Finest patterns can be written into the resist which remain on the substrate after development (E). F: To complete the the preparation, a silicon cladding layer of several $100 \mathrm{~nm}$ thickness is evaporated onto the pattern. G: SEM image of the cross section of a $2 D$ waveguide after cutting the device to the desired length.

Polymer core waveguides (see Fig. 4.9) are prepared as follows: After cleaning 
the substrate (see above), a thin PMMA film is spincoated onto the Si-wafer. For the $2 \mathrm{D}$ waveguides a drop of $18 \mu \mathrm{l}$ of the dissolved polymer $(2 \mathrm{~g} / 100 \mathrm{ml} 2$ methoxy ethyl acetate) was pipetted onto a cleaned Si wafer $\left(10 \times 10 \mathrm{~mm}^{2}\right)$ which was then spun at a rotational speed of $5000 \mathrm{rpm}$, yielding a uniform polymer layer of thickness $d=44$ $\mathrm{nm}$. The layer preparation is finished by baking it in a laboratory oven at $120^{\circ} \mathrm{C}$ for 60 minutes. In a next step, the device is further processed by EBL, where a series of different waveguide structures are written in the resist film: (i) broad areas are kept untreated to provide 1D waveguides, (ii) gratings of an overall size of several microns with a repeat distance of $300 \mathrm{~nm}$ are drawn, and (iii) several thin lines are left unexposed in-between comparatively large areas of complete exposure (see Fig. 4.10). The additional 1D waveguides and the waveguide grating are needed in
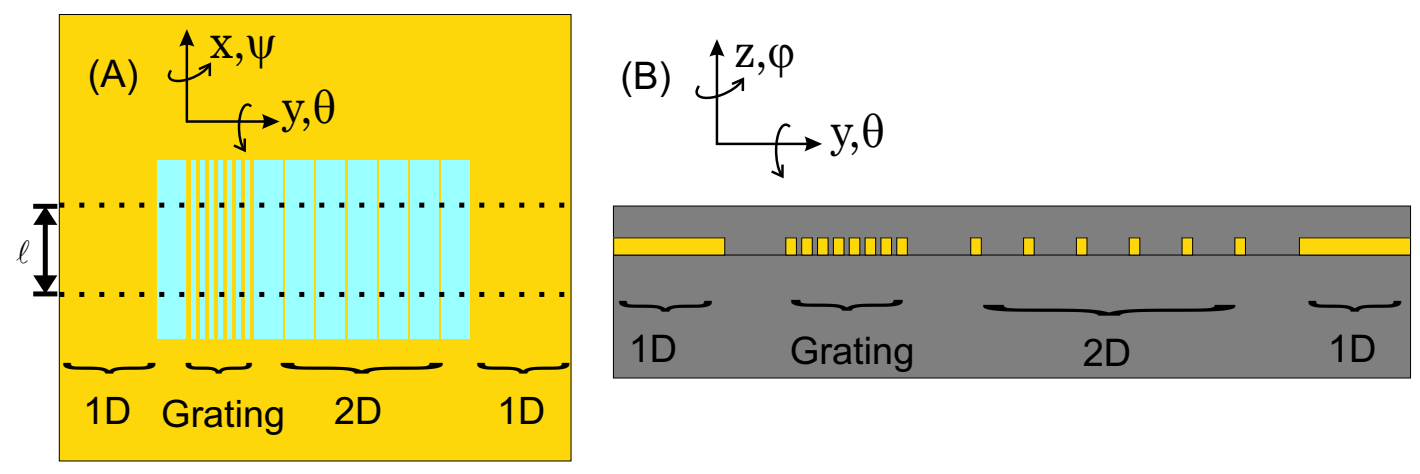

Figure 4.10: The alignment of $2 D$ waveguides requires auxiliary $1 D$ waveguides and an expanded grating made up of wide $2 D$ waveguides to facilitate the alignment of the incidence angles $\varphi_{i}, \Psi_{i}$ and $\theta_{i}$. (A) The $1 D$ waveguides result from simply not exposing broad areas to the electron beam; The $2 D$ waveguide grating is generated by writing 150 $\mathrm{nm}$ wide lines at a distance of $300 \mathrm{~nm}$ from each other; The $2 D$ waveguides require the longest exposure time: For each of them, a fine stripe of PMMA is left unexposed between broad (here: $20 \mu \mathrm{m}$ ) areas of complete exposure. The length of the lines has to be larger than the desired length $\ell$ of the waveguide, since the device has to be cut after evaporation of the silicon cladding layer, yielding a cross section as sketched in (B).

the experiment to facilitate the alignment of the incidence angles $\varphi_{i}, \Psi_{i}$ and $\theta_{i}$ of the $2 \mathrm{D}$ waveguide.

During the development of the resist, the polymer stripe height reduces from initially $44 \mathrm{~nm}$ to $30 \mathrm{~nm}$. In a final step, the waveguide is completed by evaporating a 200-300 nm thick cladding layer of silicon atop. The width of the stipes was varied from 60 to $100 \mathrm{~nm}$ providing waveguides with core diameters of the same size. 
With this preparation technique, an aspect ratio (waveguide width over length) of more than $10^{5}$ was achieved. For the x-ray experiments, the length of the guides is adapted to the respective $\mathrm{x}$-ray energy in order to achieve the desired absorption of the primary beam. 
FABRICATION OF X-RAY NANOSTRUCtURES 


\section{Chapter 5}

\section{Experimental I: White beam RBCs}

In this chapter we present the experimental results obtained at BESSY II, Berlin, where all experiments with white synchrotron radiation were performed.

\subsection{Introduction}

As shown in chapter 3, x-ray waveguides support mode propagation for a defined number of resonant modes, depending on the WG-geometry and its material composition. In the resonant beam coupling scheme, these modes are excited by aligning the incidence angle $\alpha_{i}$ of the impinging monochromatic synchrotron radiation beam. The energetic width of monochromatic x-rays usually is very small, since at most synchrotron radiation beamlines, monochromaticity is achieved by using perfect crystals with small energetic acceptance, typically in the order of a few eV $\left(\Delta E / E \simeq 10^{-4}\right)$, with the photon energy $E=h c / \lambda$. For many imaging and photon correlation experiments however, lateral coherence is more important than longitudinal (chromatic) coherence. The monochromaticity conditions can therefore be relaxed. Generalizing the concept of resonant beam coupling from monochromatic to white beam illumination allows for the analysis of x-ray waveguides in white synchrotron radiation beams (Jarre et al. 2003). In the following we describe experiments performed at BESSY II. We will see, that using the full energetic acceptance of a waveguide (see Fig. 5.1) is a suitable tool to increase the flux of a nm-sized waveguided beam. Along with the flux increase goes a convenient monochromatization to $\Delta \lambda / \lambda$ in the order of $10^{-2}$ or less. 


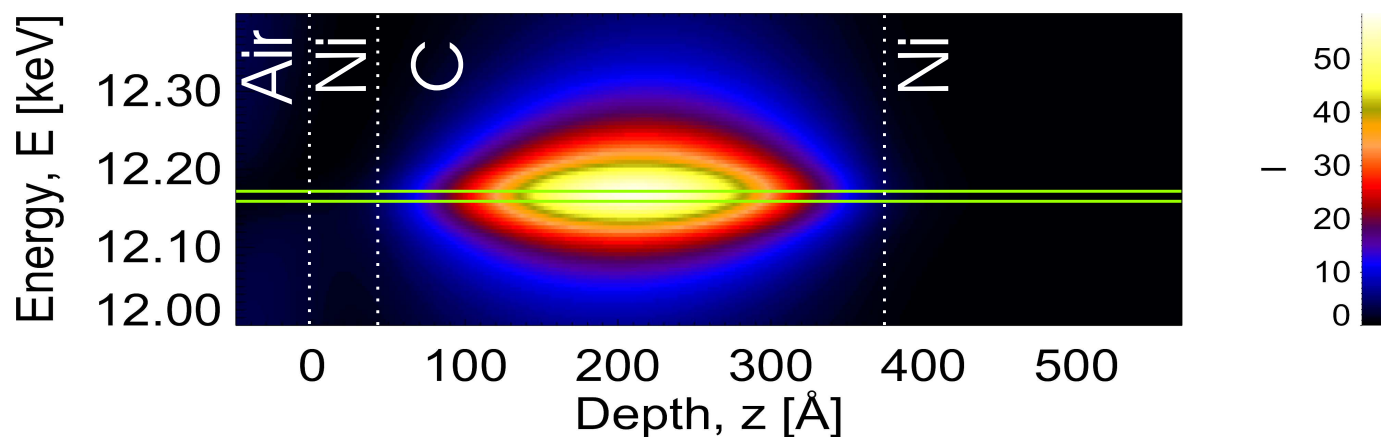

Figure 5.1: The calculated Intensity distribution of the TEO mode inside a $\mathrm{Ni} / \mathrm{C} / \mathrm{Ni}$ waveguide at a grazing incidence angle $\alpha_{i}=0.16^{\circ}$. The calculations were performed using the IMD software package (Windt. 2000). The energetic acceptance of this waveguide is larger than $100 \mathrm{eV}$, while the energetic width of most synchrotron beams, indicated by the space between the horizontal lines, is only a few eV.

\section{$5.2 \quad$ Experimental setup}

The white beam experiments presented here were performed at the energy dispersive reflectivity (EDR) beamline DIP13/2 at the $3^{\text {rd }}$-generation synchrotron BESSY II in Berlin (Pietsch et al. 2001; Panzner et al. 2003). This beamline offers the advantage, that only a minimum number of optical components, i.e. slits/pinholes, vacuum windows and absorbers, are brought into the beam path. The energy spectrum $I(E)$ of the primary beam (PB) can thus be modelled by taking into account: (i) the ring current of the BESSY II storage ring, (ii) the bending magnet emission, and (iii) the effective absorption of all elements in the beam (air, windows). The spectrum must therefore be of the form of

$$
I(E[k e V])=a e^{-b(E[k e V])-c /(E[k e V])^{3}},
$$

where a scales with the ring current, b determines the asymptotic behavior at high $\mathrm{E}$, and c relates to the effective absorption. The measured curve of the PB can be fitted to $\mathrm{b}=0.48$ and $\mathrm{c}=480$, respectively (see Fig. 5.2). A sketch of the setup used to study the properties of RBC waveguides in a white synchrotron radiation beam is shown in Fig. 5.3. The beam dimensions are set by a $10 \mu \mathrm{m}$ Pt-pinhole 350 $\mathrm{mm}$ in front of the waveguide. The waveguide is mounted on a standard HUBER goniometer to adjust the incidence angle $\alpha_{i}$, while the detector is mounted 1150 mm behind the waveguide. The energy dispersive detector (X-flash 1000, Röntec) provides an energy resolution of $200 \mathrm{eV}$ at integral count rates of up to 100000 counts per second (cps). 


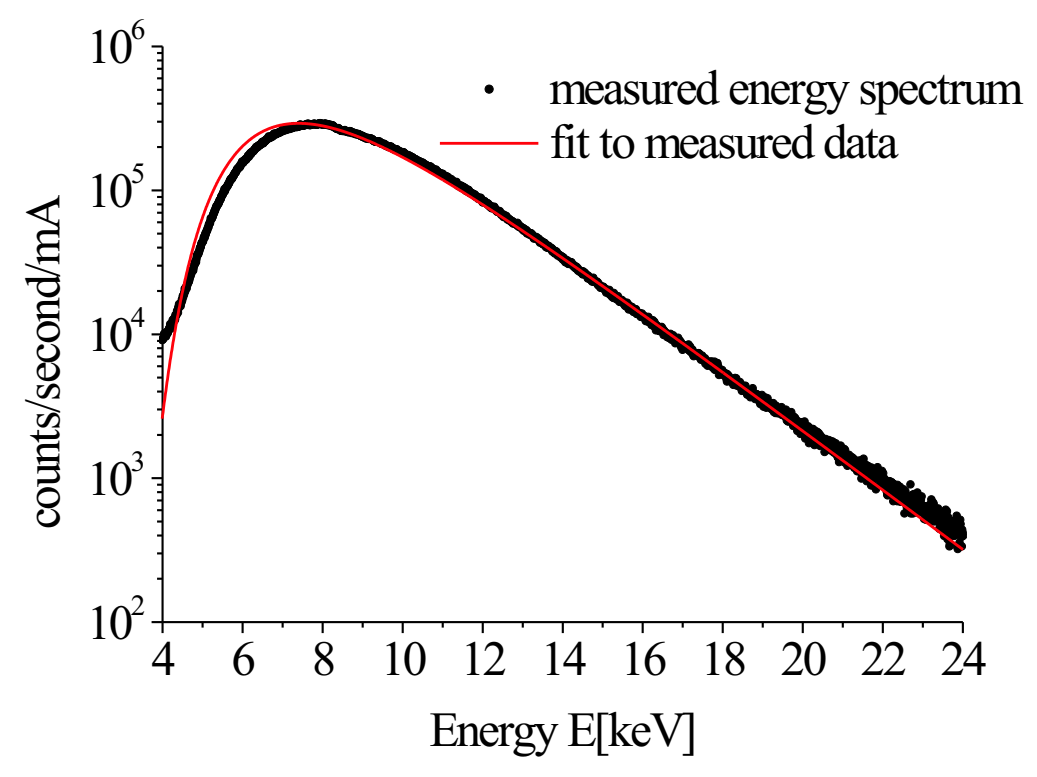

Figure 5.2: Measured primary beam spectrum (symbols) of the EDR beamline at BESSY II, Berlin and a fit to the measured data according to eq. 5.1 The curve was measured by Prof. U. Pietsch of the Potsdam University in low current single bunch mode, so no additional absorbers had to be brought into the beam path.

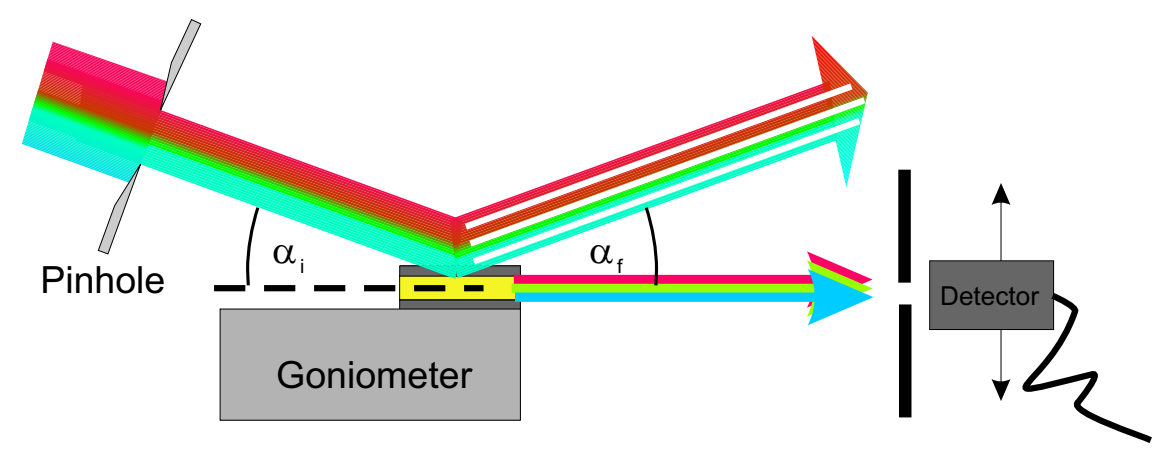

Figure 5.3: Sketch of the experimental setup at the EDR beamline: The incoming white beam, indicated by the rainbow colors, impinges on the waveguide at the grazing incidence angle $\alpha_{i}$. The device itself 'finds' all energies that fulfill the mode excitation condition. These energies are found to be missing in the specular reflected beam and can be harvested at the exit of the waveguide. 


\subsection{Experiments}

Since the white beam provides all energies that fulfill mode excitation condition at a given incidence angle $\alpha_{i}$, a set of $\mathrm{N}$ (total number of modes supported by the examined waveguide) energies will be guided in the device. In other words: the white beam illuminated waveguide automatically 'finds' all energies which correspond to resonant mode excitation at a given incidence angle. These resonant energies can be evidenced in two ways: (i) as cups in the plateau of total external reflection, and (ii) in the energetic and angular distribution of the beam exiting the device. In Fig. 5.4, we show the measured reflectivity $\left(\alpha_{i}=\alpha_{f}\right)$ together with the measured integrated farfield $\left(\alpha_{f}=0\right)$ of a $20 / 32 / 5 \mathrm{~nm} \mathrm{Ni} / \mathrm{C} / \mathrm{Ni}$ waveguide on $\mathrm{Si}$ at an incidence angle $\alpha_{i}=0.194^{\circ}$. For the reflectivity measurements, a detector pinhole of $35 \mu \mathrm{m}$ diameter was used, providing an angular resolution of $0.03 \mathrm{mrad}$. At these settings the width (FWHM) of the primary beam was measured to $27 \pm 1 \mu \mathrm{m}$ corresponding to a detector angle of $0.23 \pm 0.01 \mathrm{mrad}$. To integrate the intensity of the farfield pattern of the guided beam, the detector pinhole was replaced by a slit, set to $2 \mathrm{~mm}$, equivalent to an integration over $0.1^{\circ}(1.74 \mathrm{mrad})$.

A comparison of the measured intensities of the reflectivity curve and of the farfield pattern of the guided beam yields the gain of the waveguide, defined by the ratio of photons exiting the waveguide to the number of photons crossing hypothetical slits of the same size as the guiding layer. For the first mode we get a value of $g=40$. Note, however, that with the same gain, the white illumination yields more photons in the waveguided beam, simply because the energetic bandpass is larger than in monochromatic synchrotron beams (see Fig. 5.1).

The solutions $\kappa_{n}$ of the transcendental eigenvalue equation (Eq. 3.20) provide the energies $E_{n}$ at fixed internal angle $\alpha_{1}$, or equivalently at fixed incidence angle $\alpha_{i}$ by the relation

$$
\kappa_{n}=\frac{2 \pi}{h c} \cdot E_{n} \sin \alpha_{1} .
$$

From this equation we find that the resonant energies $\mathrm{E}_{n}$ can be tuned by changing the incidence angle. In Fig. 5.5 b) the simulated reflectivity of the waveguide described above is presented as a function of the incidence angle $\alpha_{i}$ and the energy E. For simulations of the white beam waveguide optics, we used the IMD software package (Windt. 2000). The waveguide was designed to support three modes. While the total number of guided modes is fixed, their energetic position follows the incidence angle. The simulation shows the reflectance of the device in logarithmic grayscales, where dark shades represent low reflectance values. The cusps in 

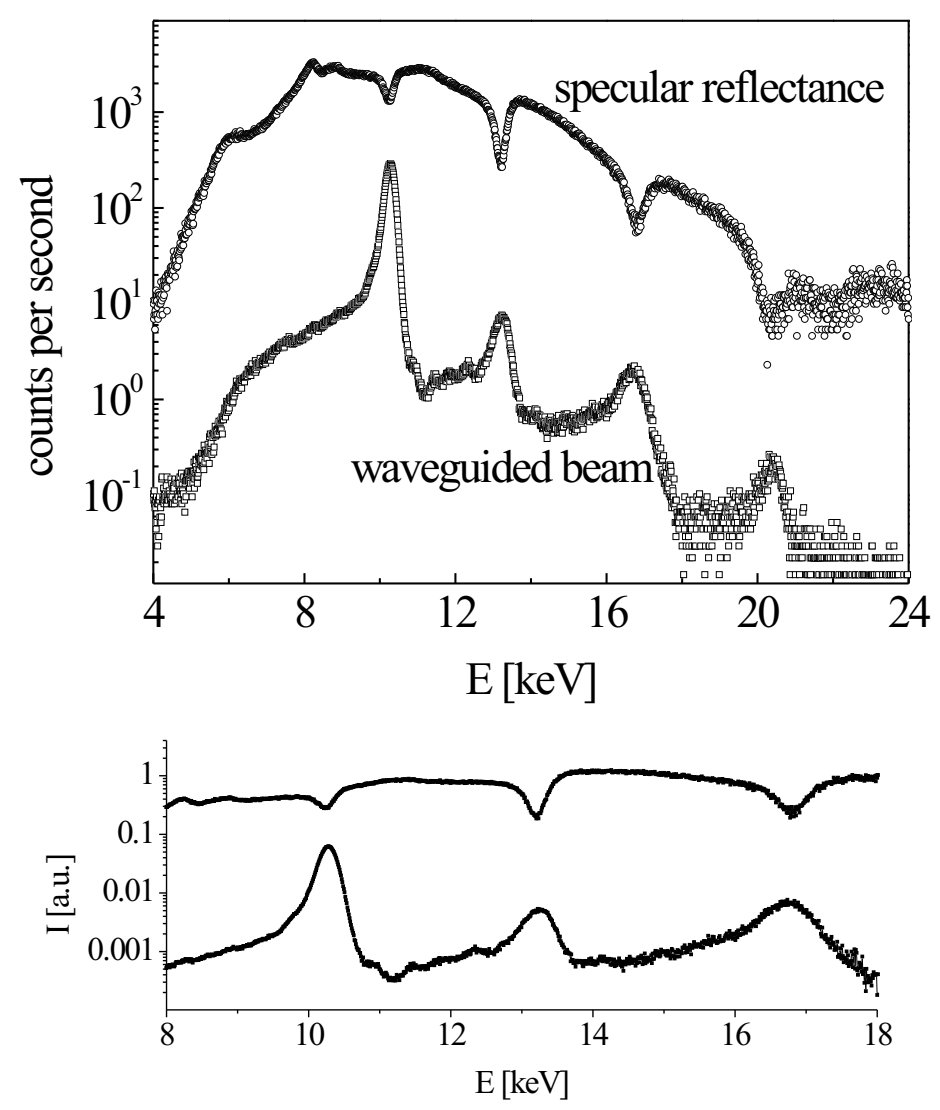

Figure 5.4: Experimental results. Top: Comparison of the specular reflected and the waveguided beam. The resonant energies (guided modes) are evidenced either as cusps in the plateau of total reflection (upper curve) or in the farfield of the beam exiting the device at its side (lower curve). Bottom: Zoom to the plateau of total reflection after normalizing the data to the fitted primary beam profile (Eq. 5.1).

the plateau of total reflection, i.e. the resonant energies, are therefore represented by the dark curved lines. Increasing the incidence angle shifts the energies of the guided modes towards smaller values. This simulation is compared to the measured data of the waveguided beam. Fig. 5.5 a) shows the energetic distribution of the guided beam, consistently as a function of the incidence angle $\alpha_{i}$ in logarithmic grayscales. Please note, that in this Figure, dark shades indicate high intensities. Therefore they are again representing the modes of the device.

As shown before, in small angle approximation the farfield distribution of the guided 

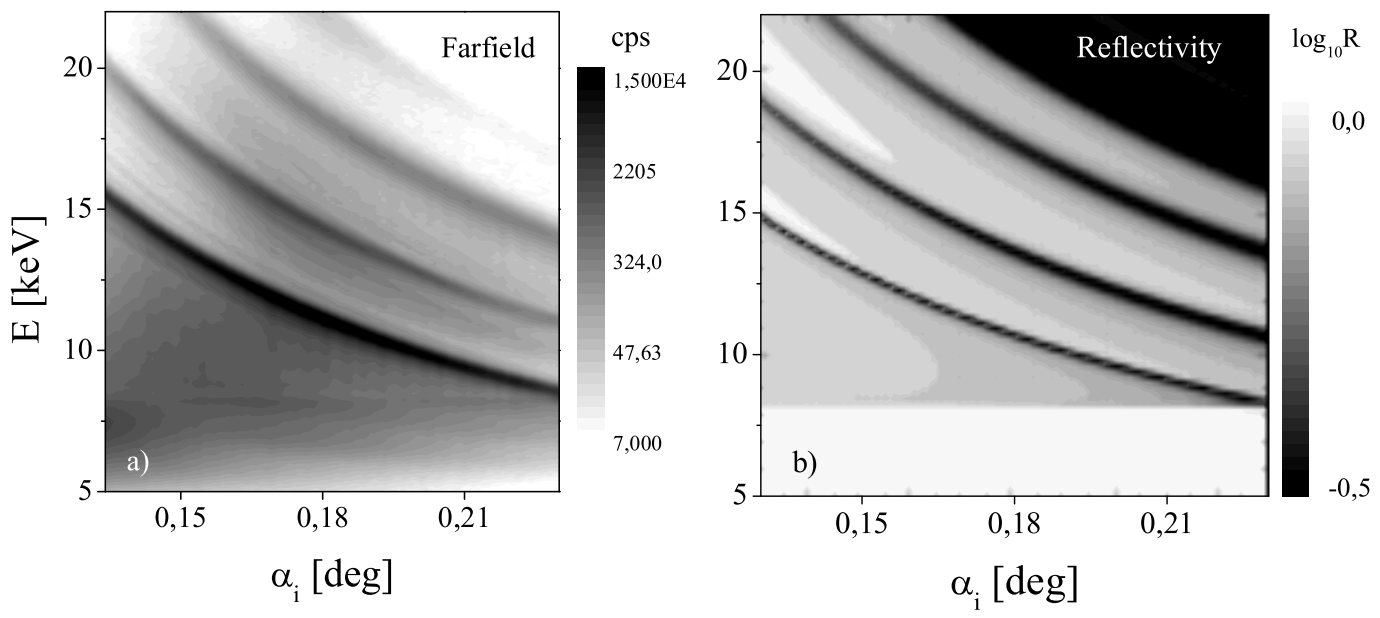

Figure 5.5: (a) The measured energetic farfield distribution of a three mode supporting waveguide as a function of the incidence angle $\alpha_{i}$ and (b) the simulation of the specular reflectivity of the same waveguide. The energetic position of both, the guided beam and the cusps in the plateau of total reflection - each identified by dark shades - are shifted by changing the incidence angle of the impinging white beam following Eq. 5.2.
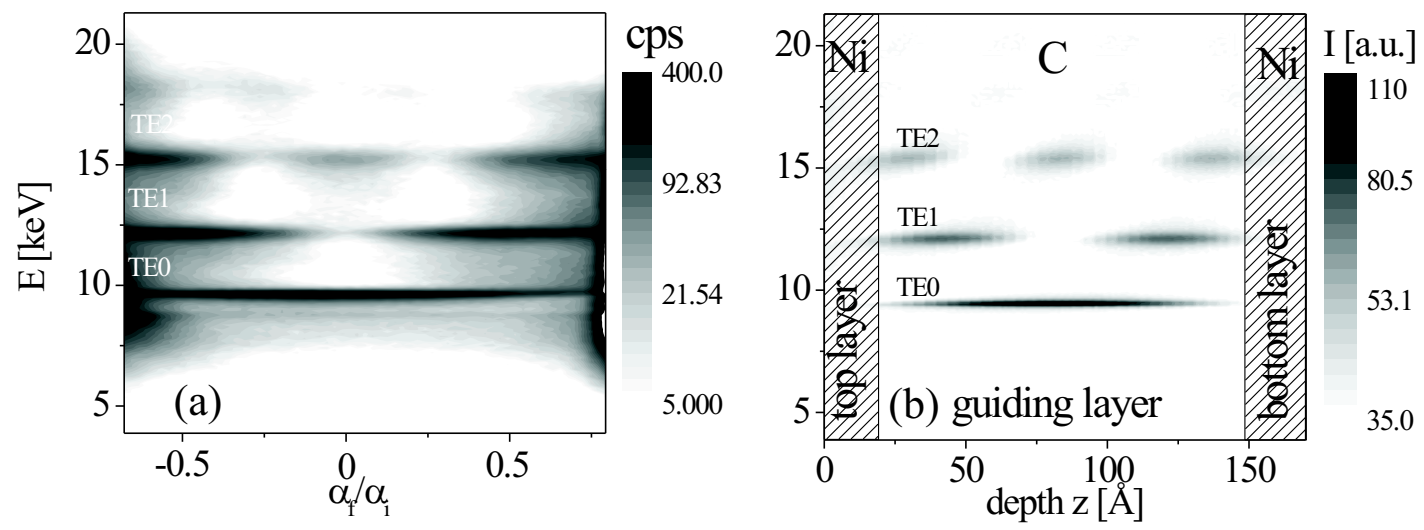

Figure 5.6: (a) The measured angular farfield distribution of the three mode supporting $1 D$-waveguide at a grazing incidence angle $\alpha_{i}=0.2^{\circ}$ and (b) the calculated intensity distribution inside the waveguide at the same incidence angle.

beam is given by

$$
\mathcal{I}\left(\alpha_{f}, E\right)=\mathcal{I}_{0}\left|\int_{-\infty}^{+\infty} \Psi(z) e^{i k_{0}(E) \alpha_{f} z} d z\right|^{2}
$$


A Simulation of the internal intensity distribution of the $20 / 32 / 5 \mathrm{~nm} \mathrm{Ni} / \mathrm{C} / \mathrm{Ni}$ waveguide and the energetic and angular distribution of the measured farfield are presented in Fig. 5.6. In order to measure the angular distribution of the modes in the farfield pattern between the transmitted PB and the specular reflected beam $\left(-\alpha_{i}<\alpha_{f}<\alpha_{i}\right)$, the detector slit was set to $0.1 \mathrm{~mm}$ corresponding to an angular resolution of $0.087 \mathrm{mrad}$. The energetic width of the mode is matched to the acceptance of the waveguide, leading to high coupling efficiencies. The simulations predict a Full Width at Half Maximum (FWHM) of the three modes of $130 \mathrm{eV}, 310$ $\mathrm{eV}$ and $580 \mathrm{eV}$ for the first, second and third mode, respectively, while the measured data exhibits a width of $240 \mathrm{eV}, 365 \mathrm{eV}$ and $700 \mathrm{eV}$. This is not an indication for a disagreement between the simulation and the experiment, since the measured data is convoluted with the detector resolution. The knowledge of the energetic width from the simulations could be used to increase the the energetic resolution in white beam experiments and to reduce the gap between the monochromaticity achieved with crystals and the energy resolution provided by modern energy dispersive detectors especially in the case of multimodal excitation.

In Fig. 5.7 we exemplarily show the angular and energetic farfield distribution of a

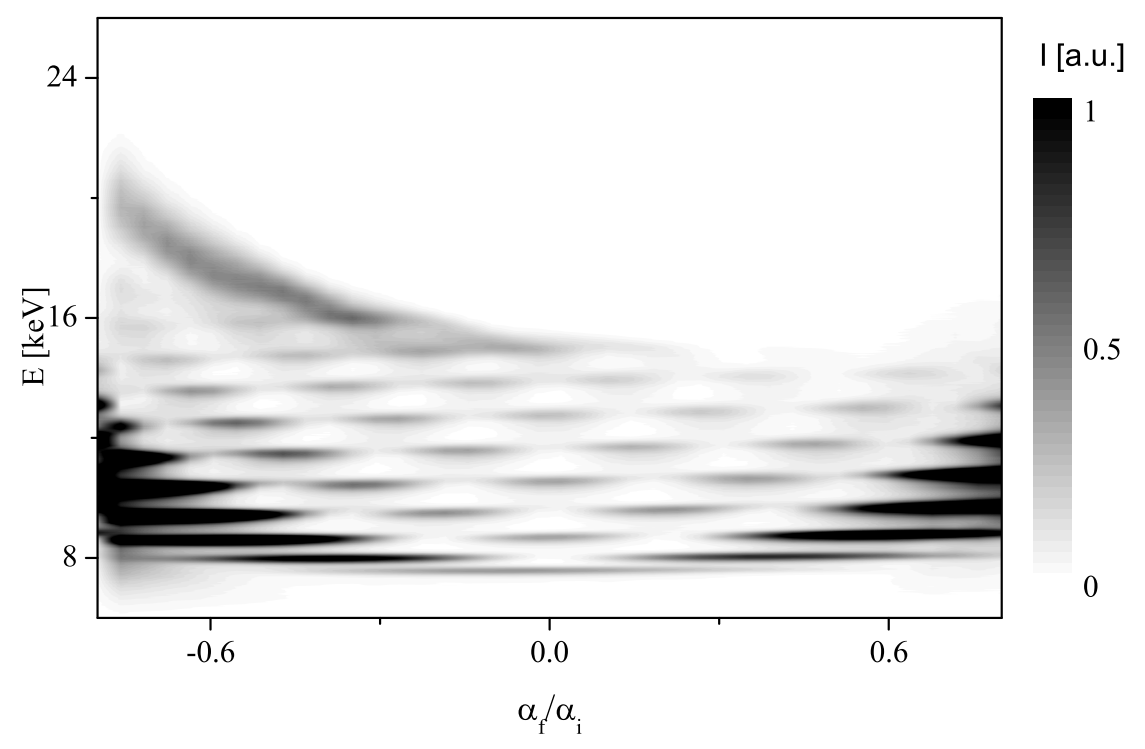

Figure 5.7: Measured farfield distribution of a multimode Cr/PMMA/Cr waveguide with a guiding layer thickness of $130 \mathrm{~nm}$ at $\alpha_{i}=0.17^{\circ}$

Cr/PMMA/Cr (50/130/6 nm) on Si multimode waveguide supporting ten modes. The energetic width of the first mode for example is calculated to $22 \mathrm{eV}$ which is 
an order of magnitude below the detector resolution of $200 \mathrm{eV}$. The width of all ten modes is given in tab. 5.3.

\begin{tabular}{|c|c|c|c|c|c|c|c|c|c|c|}
\hline modenumber & 1 & 2 & 3 & 4 & 5 & 6 & 7 & 8 & 9 & 10 \\
\hline energy $[\mathrm{keV}]$ & 7.5 & 7.9 & 8.5 & 9.3 & 10.3 & 11.2 & 12.3 & 13.5 & 14.7 & 15.9 \\
\hline width $[\mathrm{eV}]$ & 23 & 30 & 39 & 56 & 75 & 93 & 116 & 144 & 176 & 216 \\
\hline$\Delta \lambda / \lambda\left[10^{-2}\right]$ & 0.31 & 0.38 & 0.46 & 0.60 & 0.73 & 0.83 & 1.04 & 1.07 & 1.20 & 1.36 \\
\hline
\end{tabular}

Table 5.1: Relevant parameters of a ten mode supporting $\mathrm{Cr} / \mathrm{PMMA} / \mathrm{Cr}(50 / 130 / 6 \mathrm{~nm})$ waveguide at an incidence angle $\alpha_{i}=0.18^{\circ}$.

Monomodal waveguides in turn offer the advantage of a particularly clean (Gaussianlike) beam profile going along with large energetic acceptance at minimum guiding layer thicknesses providing smallest beam sizes. In Fig. 5.8 a) the reflectivity and the guided beam are shown measured at an incidence angle $\alpha_{i}=0.12^{\circ}$. The right graph (b) shows the farfield pattern of the guided beam for five different incidence angles: (from blue to green) $\alpha_{i}=0.11,0.12,0.13,0.14$ and $0.15^{\circ}$. This $\mathrm{Ni} / \mathrm{C} / \mathrm{Ni}$ waveguide exhibits a guiding layer thickness of $10 \mathrm{~nm}$ only. With $1.19 \mathrm{keV}$ (FWHM) the energetic bandwidth of this device is particularly large providing high flux at small beamsize - the exiting beam dimension in vertical direction is 9nm FWHM only! 

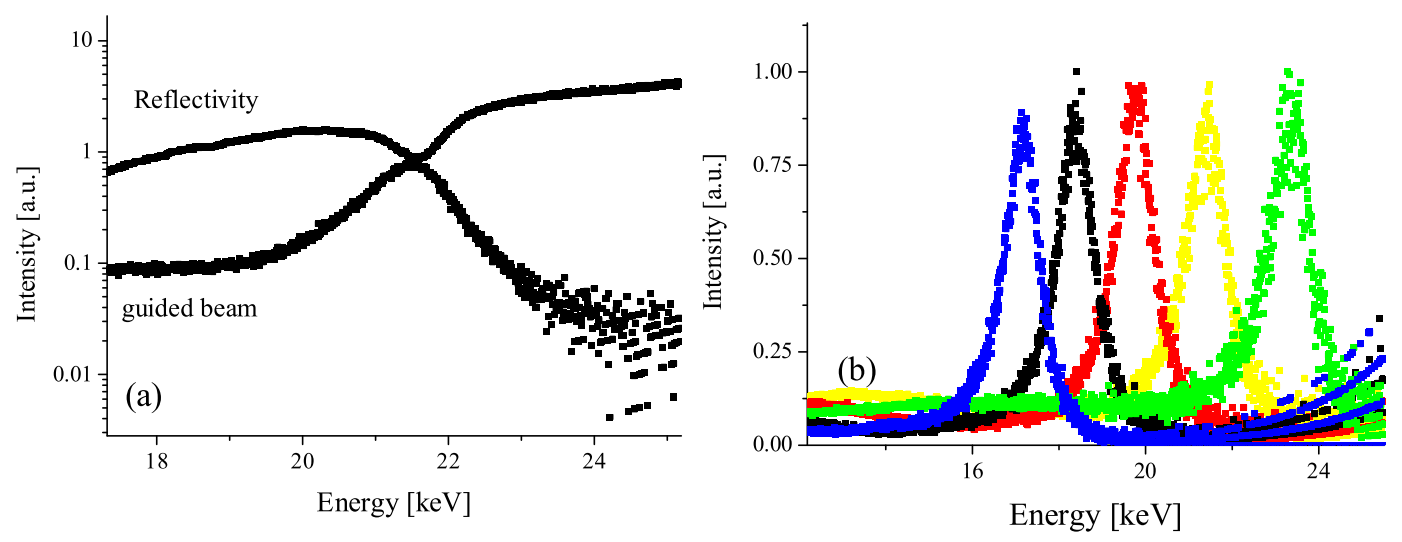

Figure 5.8: a) Measured reflectivity $\left(\alpha_{i}=\alpha_{f}\right.$ and exiting beam $\left(\alpha_{f}=0\right)$ ) of a $\mathrm{Ni} / \mathrm{C} / \mathrm{Ni}$ $(50 / 10 / 6 \mathrm{~nm})$ waveguide at $\alpha_{i}=0.14^{\circ}$ and (b) the exiting beam profiles for incidence angles between $0.11^{\circ}$ (blue) and $0.15^{\circ}$ (green). 
EXPERIMENTAL I: White BEAm RBCs 


\section{Chapter 6}

\section{Experimental II: 2D-Waveguides}

$X$-ray waveguides in the resonant beam coupling (RBC) scheme have been investigated by several groups since the late nineteen seventies, mainly in the case of one dimensional beam confinement (planar waveguides). Two dimensional (2D) compression has first been achieved by Pfeiffer et al. (2002). However, the exiting waveguided beam was of rather low intensity and accompanied by spurious reflected and primary beams. Therefore we have applied a new coupling scheme for two dimensionally confining waveguides in order to optimize $x$-ray waveguides for future applications.

\subsection{Introduction}

In the front coupling regime developed for two dimensional confinement of hard $\mathrm{x}$-ray beams, the waveguide acts as a spatial and coherence filter. An important characteristic of these devices is their ability to block all the unwanted radiation of the primary beam, yielding a well defined nano beam with a divergence of a few mrad. While high aspect ratios are needed to achieve the desired absorption of the primary beam, the necessary flux enhancement is provided by an external focusing optic. On the following pages we will describe the experiments and the results obtained during several beamtimes at HASYLAB/Hamburg and at the ESRF/Grenoble.

\section{Experimental setup}

Since the experiments presented in this chapter were performed at different 


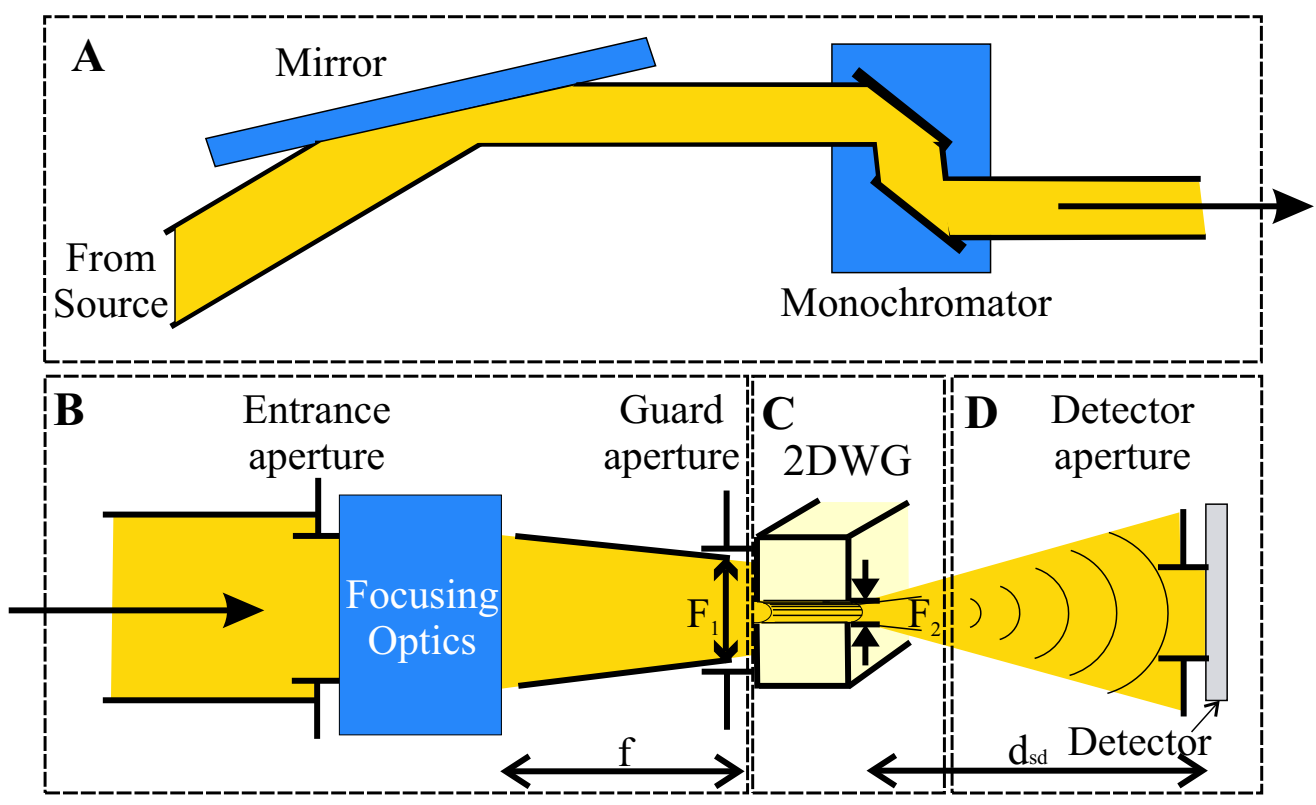

Figure 6.1: Sketch of the general beamline setup for experimental examination of two dimensionally confining waveguides. It is divided into (A) beam preparation, (B) a focusing section, $(C)$ the waveguiding section and $(D)$ the detection of the guided beam.

beamlines and synchrotrons, a more general description of a typical setup is given in the beginning to sketch all components used. Fig. 6.1 illustrates this setup, which can be divided into four more or less independent parts: (A) The x-ray-source characterized by the energetic, spatial and angular dimensions of the primary beam (slits, mirrors, attenuators, monochromators), (B) the focusing optic (introduced in chapter 2), (C) the waveguide (see chapter 4) and (D) the detector. More detailed information on the setup of each experiment is given in the respective sections of this chapter.

\subsection{FIB-fabricated waveguides and silk templates}

The experiments on 2D waveguides fabricated with focused ion beam (see chapter 4.2 ) were performed at the ID18f undulator beamline at the ESRF. The x-ray energy was set to $E_{1}=8 \mathrm{keV}$ by a $\mathrm{Si}$ (111) double monochromator yielding a transmission through a $20 \mu \mathrm{m}$ tantal foil of $T_{1}=4.7 \cdot 10^{-3}$ (see Fig. 6.2). In order 


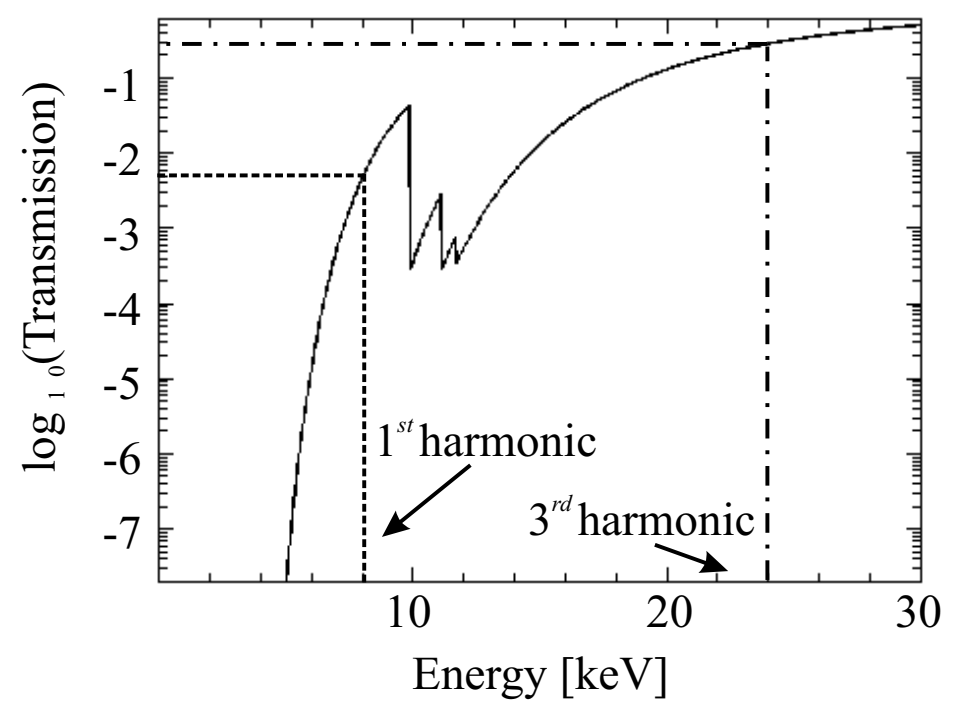

Figure 6.2: Transmission of a $20 \mu \mathrm{m}$ tantal foil on double logarithmic scales. The experiment was performed at an x-ray energy of $8 \mathrm{keV}$ (dashed line). The third undulator harmonic is therefore at $24 \mathrm{keV}$ (dash-dot).

to reduce higher harmonics from the undulator (the third harmonic at $E_{3}=24$ $\mathrm{keV}$ yields a transmission of $T_{3}=0.29$ ) the second monochromator was detuned by two thousandth of a degree. At this beamline, a mirror for further reduction of high energy contributions was not available. The incoming beam was focused by a fresnel zone plate (lens material: $\mathrm{Au}$, thickness $1.5 \mu \mathrm{m}, \mathrm{N}=615$, outermost zone width: $0.25 \mu \mathrm{m})$ to $1.4 \times 22 \mu \mathrm{m}^{2}$ in vertical and horizontal direction respectively at a focal distance $f=1011 \mathrm{~mm}$ behind the lens as measured by knife edge fluorescence scans of the $\mathrm{Cr} L_{\alpha}$-edge . An order sorting aperture (OSA) made of Pt with a diameter $d=50 \mu \mathrm{m}$ was placed right in front of the waveguides to reduce the background and for further reduction of high energy contributions ${ }^{1}$. As discussed in section 4.2, the waveguides in this experiment lacked radial symmetry (an SEM image of such a structure is shown on page 49), so the far field patterns were barely predictable. Nevertheless, the experiment could prove in principle the possibility of front coupling of a focused hard x-ray beam into a 2D waveguide. As we can not determine the size of the drilled hole in the foil, but only at its entrance and exit, the number of supported modes can not be determined. But it is expected that for

\footnotetext{
${ }^{1}$ The first order focal length of the third harmonic $f_{1}\left(E_{3}\right)$ is three times the first order focal length of the first harmonic $f_{1}\left(E_{1}\right) \cdot f_{1}\left(E_{3}\right)=3 \cdot f_{1}\left(E_{1}\right)$, see Fig. 2.1
} 

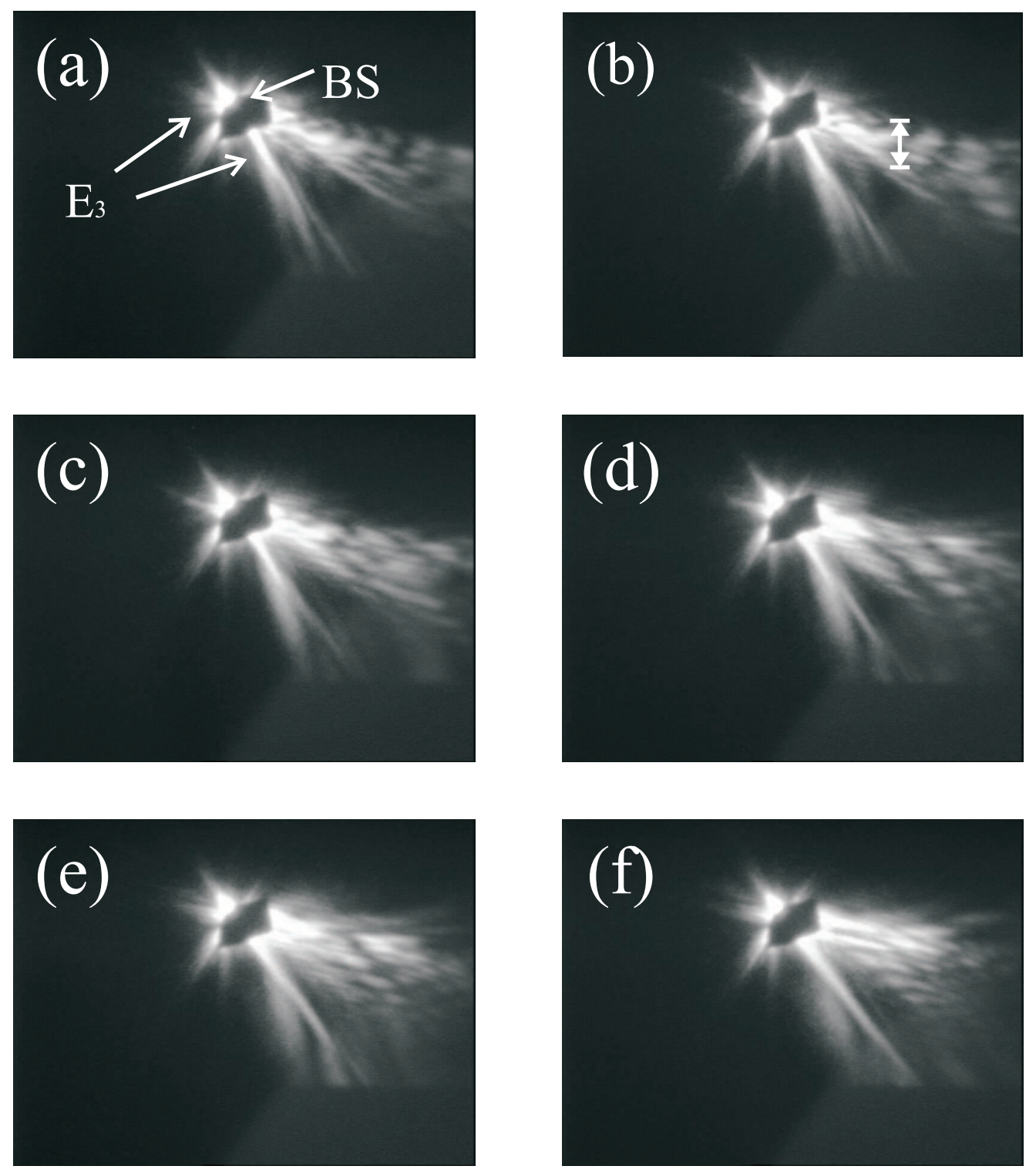

Figure 6.3: The farfield pattern of the 2d waveguide shown on page 49 at incidence angles between nominally -0.45 and 0.3 degree. Due to the non sufficient suppression of the third harmonic a Beamstop $(B S)$ is needed to protect the camera. High energies $\left(E_{3}\right)$ are not sensitive to a variation of the incidence angles while the lower energy radiation exhibits features characteristic for mode mixing in two dimensional confining waveguides and is therefore highly sensitive on changes of the incidence angle. 
these rather large structures and the high contrast of the refractive indices of air (guiding core) and Ta (cladding), N must be large: A comparable one dimensionally confining guide with an 'air-layer' thickness of e.g. $300 \mathrm{~nm}$ supports more than thirty modes. Therefore, due to mode mixing in the guiding core, a speckle-like pattern in the farfield, highly sensitive to the incidence angle can be expected. The experimental observation of this pattern can therefore give evidence for successful front coupling a focused hard x-ray beam into a 2D waveguide. The far field patterns were recorded using an X-ray Eye camera (Photonic Science, Millham, UK) with an active area of $14.0 \mathrm{~mm} \times 10.5 \mathrm{~mm}^{2}$, corresponding to farfield angels of 0.91 and 0.68 degrees respectively at a sample-detector distance of $d_{s d}=440 \mathrm{~mm}$. In order to block the high energy contributions of the transmitted primary beam as much as possible, a lead made beamstop (BS) was mounted directly in front of the camera. Fig. 6.3 shows the recorded far field pattern at different incidence angles $\alpha_{i}$. These images show that high energies dominate the far field pattern. Their contribution to the far field does not change with the incidence angle, while the farfield of the $8 \mathrm{kev}$ radiation does. It is the farfield pattern of the waveguided beam and exhibits the expected multimodal behavior. The $2 \mathrm{D}$ detector is characterized by a poor discrimination between high and low photon energies. A better suppression of high harmonic detection can be achieved using a scintillation detector. Therefore, the farfield pattern was also examined with a Cyber star (Oxford instruments) which was electronically set to the detection of $8 \mathrm{keV}$ radiation (SCA - window). Fig. 6.4 shows line scans along the axis shown in Fig. 6.3 (b) at incidence angles between 0.15 and 0.6 degree, demonstrating the sensitivity of the $8 \mathrm{keV}$ farfield due to mode mixing in the guide. While the experiment was successful concerning the principle possibility of coupling a focused beam into an x-ray waveguide, it also demonstrates that it is very hard to fabricate reasonable geometries with a focused ion beam.

The waveguides which are fabricated by templating from spider silk offer a (nearly perfect) cylindrical geometry and are therefore interesting candidates. Furthermore these devices offer the advantage that they can be fabricated with larger diameters in the range of a micron which allows for experimental examination also at $2^{\text {nd }}$ generation synchrotron sources. A selection of different devices which were tested at the D4 bending magnet beamline at the Hamburger Synchrotron Strahlungslabor (HASYLAB) is shown on page 54 . The beamline yields a photon flux of $\sim 10^{9}$ photons per second per $\mathrm{mm}^{2}$. Assuming an efficiency of the waveguide (number of photons hitting the waveguide entrance over number of photons exiting the device) 


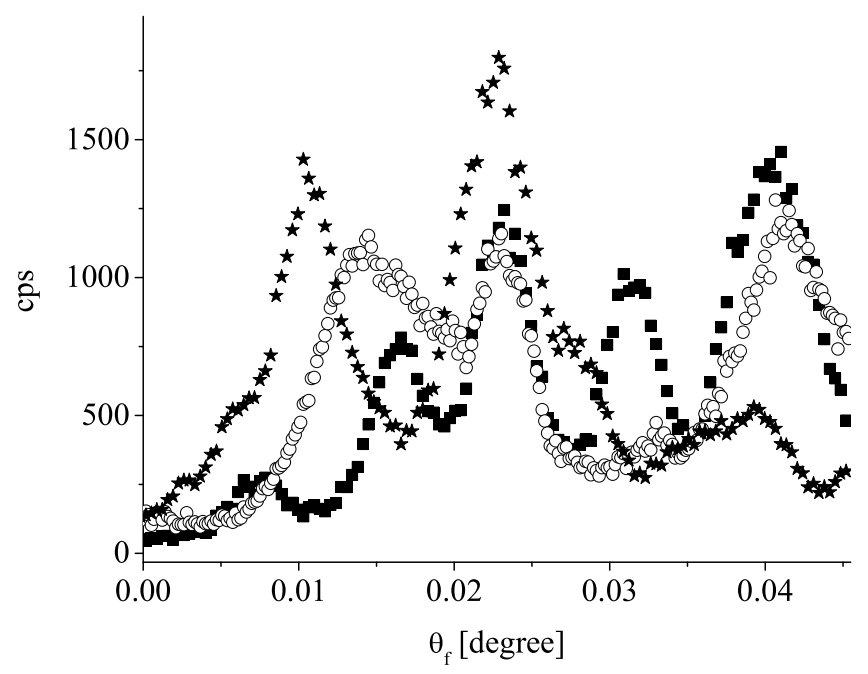

Figure 6.4: Far field scans of the Ta-foil waveguide at incidence angles 0.15 (solid squares), 0.3 (open circles) and 0.45 (stars) degree.

of $5 \%$, a fiber with a cross section of $1 \mu \mathrm{m}$ should guide at least 50 photons per second exiting the guide.

Monochromaticity was achieved using a Ge (111) crystal set to x-ray energies between eight and eleven keV during several beamtimes, and high energy suppression was achieved by a tilted Rh-mirror in the beam path. The detector used at this beamline was a Cyber Star single photon counter (Oxford Instruments). While the setup of the experiment concerning both the beam and the waveguide seemed promising, not even a single photon was detected exiting the examined guides. Improvements concerning background suppression (by applying additional lead shields around the beam) and the alignment of the device did not improve the success of these experiments, so at some point this attempt was cancelled although there is no satisfactory explanation found for the failure of these attempts. Despite the fact that the spider silk project seemed to provide nice and elegantly fabricated structures, the lithographic fabrication of hard x-ray waveguides was pushed at the same time and it turned out to be the most successful fabrication technique for hard x-ray waveguides. The results achieved with these devices are described in the following. 


\subsection{Lithographically fabricated waveguides}

Given in principle the feasibility of coupling a focused hard $x$-ray beam into a two dimensionally confining waveguide, the challenging task is to control the guiding channels geometry by lithographically fabricated structures. In this chapter a description of the experiments with these devices is given.

\subsubsection{V-shaped guides held in a bench vice}

The preparation of V-shaped grooves in Silicon has been described in chapter 4 . In order to make waveguides based on these devices, they were cut to four $\mathrm{mm}$ and cleaned afterwards in ultrasonic bathes of acetone and ultrapure water subsequently to remove small $\mathrm{Si}$ and dust particles which adsorb on the surface while the wafer was cut. An second unstructured piece of Si was cut to the same length and cleaned afterwards as well. Both devices were dried under nitrogen flow and put together immediately afterwards. Fig. 6.5 shows a zoom onto the front of a waveguide prepared like this. While the front side is very rough-textured, it seems that the two surfaces are in intimate contact, which we will see is not the case. A $300 \mathrm{~nm}$ wide $\mathrm{V}$-groove is shown in Fig. 6.5 (C) and (D). The bright horizontal line in this SEM image is due to electrostatic charges of the upper wafers edge, which stands a little bit above the lower wafer.

These devices were investigated at the ID01 undulator beam line at the ESRF/Grenoble.

There was no focusing optic to provide flux enhancement of the impinging beam which was set to $19.90 \mathrm{keV}$ by a Si (111) double-crystal monochromator. Higher harmonics were suppressed by a tilted Rh-mirror. The far field of the waveguides was recorded with a CCD detector (Princeton TE-CCD-1242-E-1-UV) $435 \mathrm{~mm}$ behind the guide. A typical far field image of these devices is shown in Fig. 6.5 (E). We can identify the transmitted primary beam (PB), a 1DWG farfield, and a weak signal of scattered intensity from the V-grooves embedded into the 1DWG. Obviously there still is an unwanted gap between the wafers which can only be eliminated by bonding the two Si-pieces. 

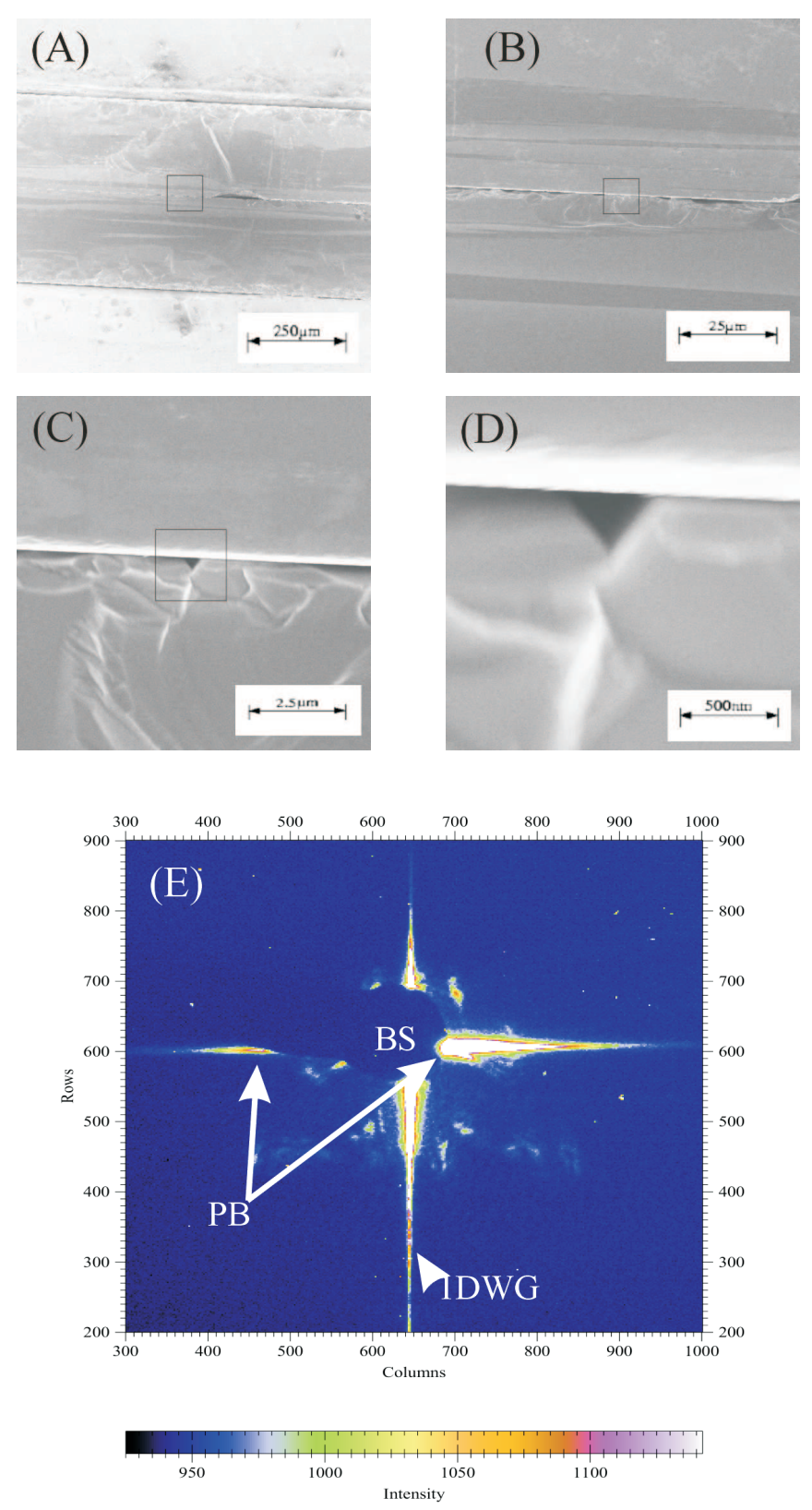

Figure 6.5: (A-D) Two Si-wafers on top of each other in a small bench vice as seen by $S E M$. One of the wafers was prepared with $V$-shaped grooves. The black squares indicate the dimension of the following image. (E) The farfield pattern of the V-groove device with the transmitted primary beam (PB), the camera protecting beam stop (BS) and the scattered signal from the v-grooves. 


\subsubsection{Experiments with a bonded grating}

Experiments with bonded waveguides were performed using U-shaped grooves. For these experiments, a grating of 300 separate U-grooves (width $w=67 \mathrm{~nm}$ ) at a distance $d=250 \mathrm{~nm}$ from each other (Seeger. 2004) was tested at the D4 bending magnet beamline at HASYLAB, Hamburg. A grating was used to test this fabrication technique, since there is no focusing optic at this beamline and the necessary flux needed for the investigation of $2 \mathrm{D}$ waveguides can only be warranted for WG gratings.

The photon energy of the synchrotron radiation beam was set to $8.94 \mathrm{keV}$ by a Ge (111) crystal as measured at the $\mathrm{Yb} \mathrm{L}_{3}$ fluorescence line. Higher harmonics were suppressed by a Rh-mirror tilted to $0.25^{\circ}$. The length $l=3 \mathrm{~mm}$ of the bonded $\mathrm{Si}$ waveguides corresponds to a transmission of the primary beam of $T=3 \cdot 10^{-14}$. The far field interference pattern of the waveguide grating was examined with a

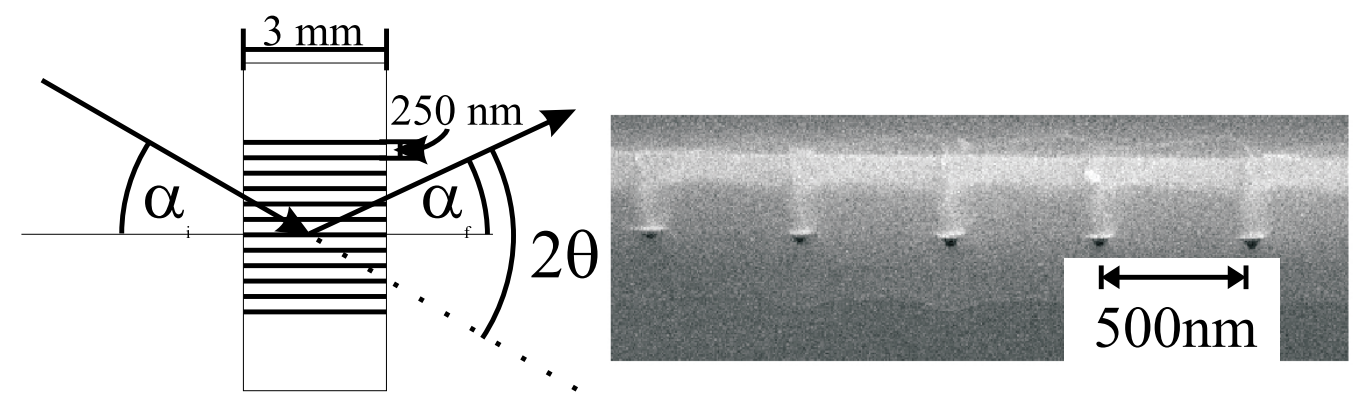

Figure 6.6: left: Sketch of the bonded U-groove grating with the angles used to describe the farfield pattern. right: Front view of a waveguide V-groove grating with a repeat distance of $500 \mathrm{~nm}$.

scintillation detector (Cyber star, Oxford instruments) $679 \mathrm{~mm}$ behind the guides. Figure 6.6 gives an overview of the angles used to describe the farfield, while Fig. 6.7 shows five of the measured far fields patterns for incidence angles $\alpha_{i}$ between $-0.04^{\circ}$ and $0.04^{\circ}$. The grating farfield is well known, providing peak intensities according to the relation $d \sin (2 \theta)=n \cdot \lambda$ i.e. at $n \cdot 0.318^{\circ}$. This is convoluted with the farfield intensity distribution of a single waveguide which represents the envelope function of the overall far field (Ollinger et al. 2005). More generally spoken, the waveguides illuminate only those grating peaks which are inside the waveguide farfield pattern. The lines in the graphs represent a fit to the measured data according to Eq. 3.53 

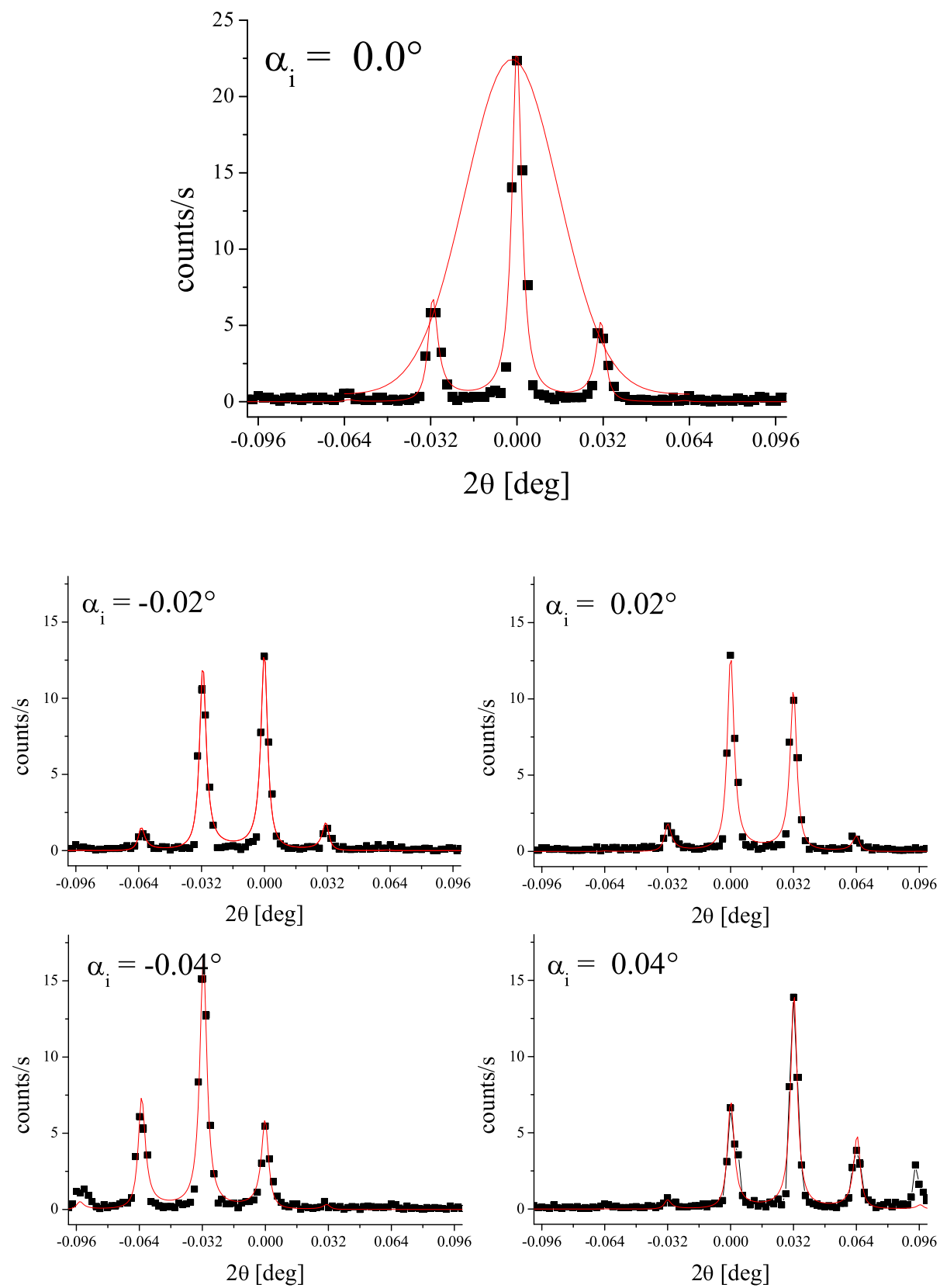

Figure 6.7: Farfield scans of a grating made up of bonded 2DWGs. For different incidence angles, the envelope of the grating farfield represents the farfield of a single waveguide. While the peak positions do not change, at each incidence angle, different peaks are illuminated. 
using a Levenberg-Marquarrdt algorithm. The fitpatameters obtained are in good agreement with the expected values: $\mathrm{d}_{\text {fit }}=250,4 \pm 0.2 \mathrm{~nm}$ and $w_{\text {fit }}=69.9 \pm 0.8$.

\subsubsection{Polymer core waveguides}

The experiments with polymer core waveguides were carried out at the ID22 undulator beamline at the ESRF/Grenoble (Jarre et al. 2005). The x-ray energy was set to $12.5 \mathrm{keV}$ by a Si (111) double monochromator. Higher harmonics of the undulator were suppressed by $(i)$ detuning of the monochromators, (ii) a Si mirror tilted to $0.14^{\circ}$ (see Fig. 6.8) and (iii) by the adaptive Rh-coated KB optics (see Fig. 6.9, chapter 2, Dabin et al. (2002) or directly at the ESRF homepage) which was used to pre-focus the incident beam onto the waveguide entrance. An aper-

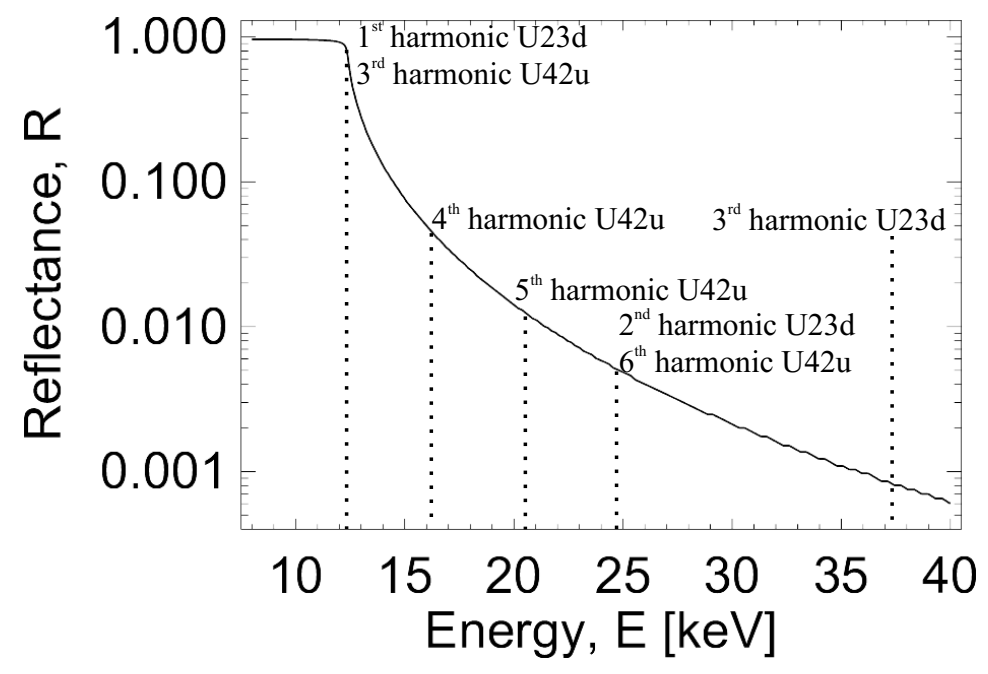

Figure 6.8: Reflectivity of the tilted Si mirror at grazing incidence angle $\vartheta=0.14^{\circ}$ used to suppress higher harmonics contributions generated by the two undulators used at the ID22 beamline (U23d and U42u).

ture of $1 \times 1 \mathrm{~mm}^{2}$ defined the incident beam in front of the KB mirrors. In order to reduce the background a $d_{\text {pin }}=20 \mu \mathrm{m}$ pinhole was mounted between the $\mathrm{KB}$ optics and the waveguide. Different detectors were used during the experiment: A fast scintillation Detector (Cyberstar, Oxford instruments) placed $470 \mathrm{~mm}$ behind the focus, a CCD system (TE/CCD-1242-EM/1, Princeton Instruments) placed $170 \mathrm{~mm}$ behind the focus, as well as a semiconductor fluorescence detector and a diode to measure the integrated flux at all positions of the setup shown in Fig. 6.1. 

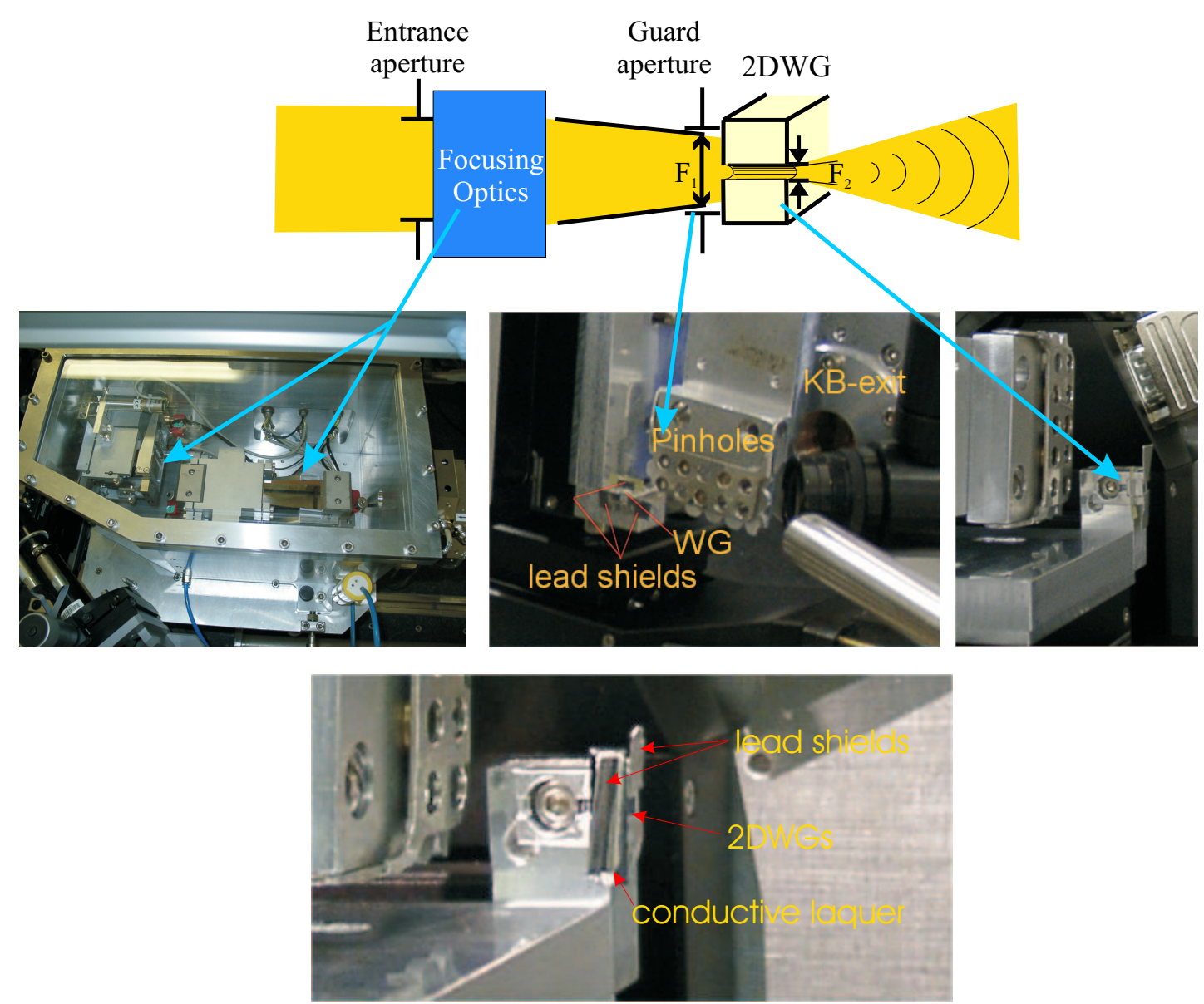

Figure 6.9: top: For the polymer core waveguide experiments, the incoming beam is focused by an adaptive KB optic (middle, left) which is held under argon flow to reduce the heatload for the mirrors and to minimize their oxidation. middle: After the beam passes the guard pinholes it hits the waveguide entrance. To prevent spuriously reflected beams from passing above or below the guide, lead shields need to be applied. The waveguide itself is comparatively tiny and hard to see. right: Top view of the waveguide holder with the guide. bottom: Enlarged view of the waveguide.

The length of the waveguide structure was $l=4.05 \mathrm{~mm}$ yielding a transmission of the primary beam ${ }^{2}$ of $T=1.8 \cdot 10^{-7}$. To block the primary beam passing above the evaporated Si cladding, an additional stripe of lead was glued atop the guide with silver lacquer. After alignment of the device, vertical linescans were performed to detect the individual 2D waveguides, see Fig. 6.10.

All count rates given in the following characterization of the waveguided beam are

\footnotetext{
${ }^{2}$ see e.g. http://www.cxro.lbl.gov/
} 


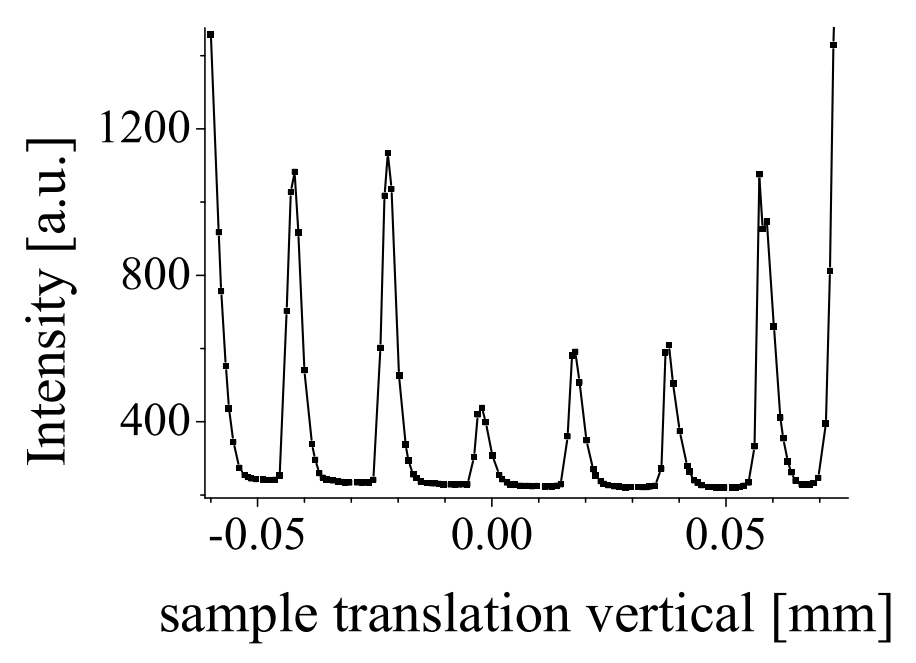

Figure 6.10: A vertical linescan of the synchrotron radiation beam across the front side of the waveguide device (see Fig. 4.10) exhibits the guided beam of the individual polymer core waveguides.

scaled to a ring current of the ESRF storage ring of $180 \mathrm{~mA}$ : The intensity of the primary beam was measured to $7.7 \cdot 10^{11}$ photons $/ \mathrm{s} / \mathrm{mm}^{2}$ directly in front of the $\mathrm{KB}$ optics, which in turn provided $3.3 \cdot 10^{11}$ photons per second in a spot size of $2.5 \times 3.8$ $\mu \mathrm{m}^{2}$ (vertically $\times$ horizontally), as measured by knife edge fluorescence (Au L-edge) scans. The entrance of the $2 \mathrm{D}$-waveguide $\left(30 \times 70 \mathrm{~nm}^{2}\right.$ [vertical $\times$ horizontal], see Fig. 6.11) was thus over-illuminated by a factor of $\sim 4.5 \cdot 10^{3}$, i.e. $7.3 \cdot 10^{7}$ photons/second $(0.02 \%$ of the focussed beam) hit the waveguide entrance. Behind the device, $3.5 \cdot 10^{6}$ photons/second were detected, corresponding to an efficiency of the waveguide of $4.7 \%$ (number of photons hitting the waveguide entrance over number of photons exiting the device). Compared to the intensity of the unfocused primary beam, the flux density of the beam exiting the waveguide is enhanced by a factor $g=4000$.

Fig. 6.12 shows the simulated intensity distribution inside the waveguide in logarithmic gray scales. The simulation predicts an efficiency of the device of $5.6 \%$, which is slightly more than the measured value. This can be explained by taking into account the convergence of $0.5 \mathrm{mrad}$ (horizontal) and $0.8 \mathrm{mrad}$ (vertical) of the incident beam. From the angular distribution of the farfield of the waveguide we can estimate an angular acceptance of the guide of $\sim 80 \%$ for these focusing angles. The agreement between experimentally obtained count rates and the 


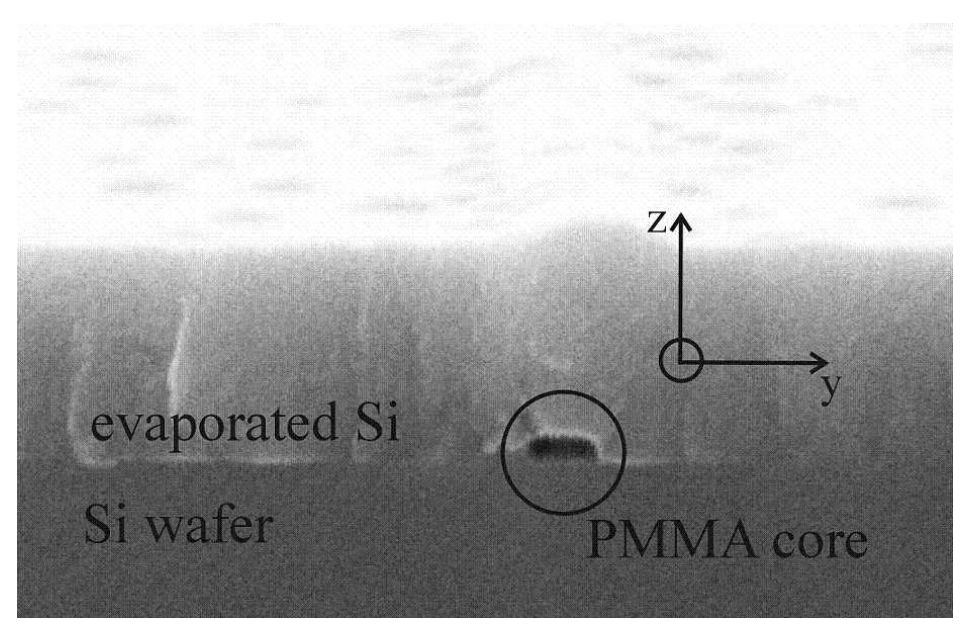

Figure 6.11: Entrance of a $30 \times 70 \mathrm{~nm}^{2}$ waveguide. The guiding core is made of PMMA. The structure was fabricated using spin coating, e-beam lithography and electron beam evaporation techniques before cutting it to its final length $\ell=4.05 \mathrm{~mm}$.

simulated intensity is then within $5 \%$. The entirely different optical properties of

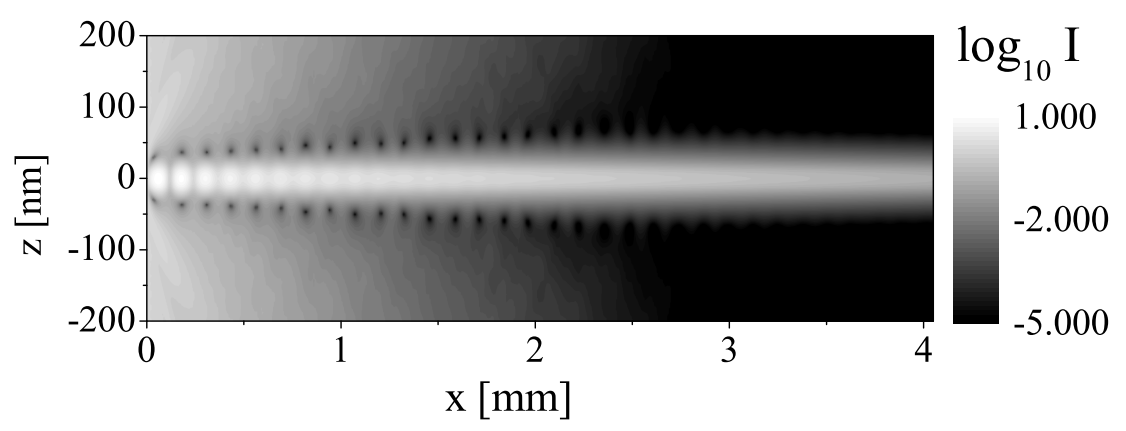

Figure 6.12: Simulated intensity distribution inside a $30 \times 70 \mathrm{~nm}^{2}$ polymer core waveguide calculated for an $x$-ray energy of $12.5 \mathrm{keV}$ in logarithmic gray scales.

the beams produced by (i) only the KB system, and (ii) the filtered beam produced by the combined optics is illustrated by the CCD images obtained in the far-field, see Fig. 6.13. While the KB image shows small scale interference fringes, such a fine structure is not observed in the waveguided beam. In the latter case the profile is particularly clean along the vertical axis reflecting the mono-modal behavior achieved due to the small waveguide height, while the horizontal profile shows some intensity variation indicative of multi modal excitation. The respective 


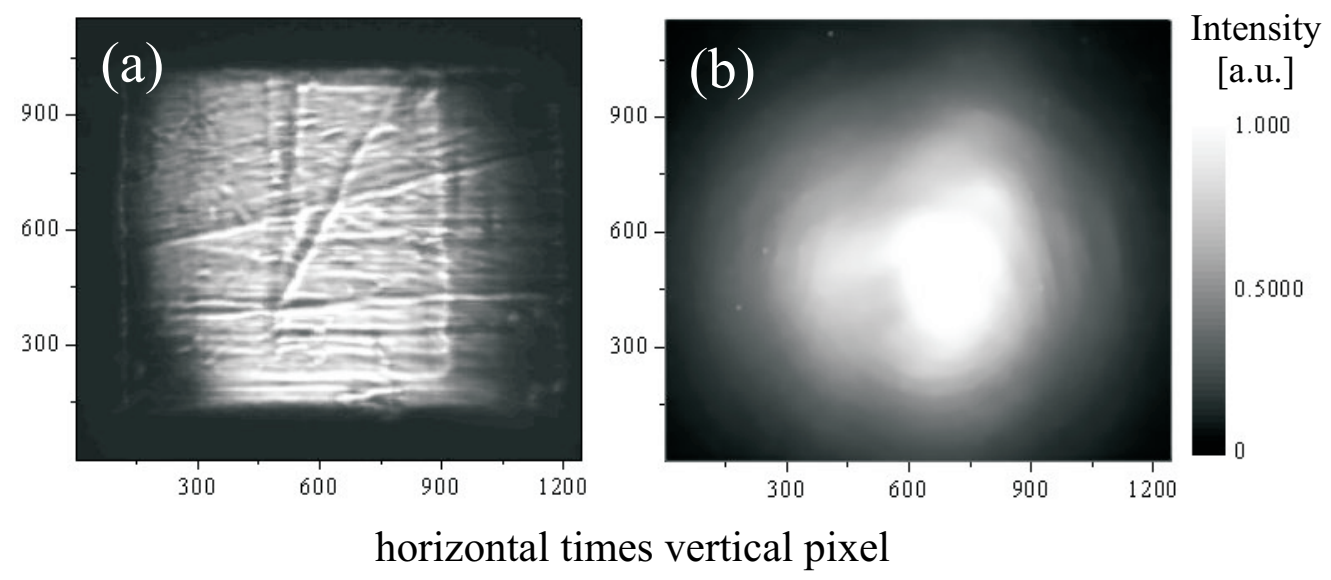

Figure 6.13: CCD images of the beam defined (a) by only the Kirkpatrick-Baez focussing optics and (b) by the combined focussing and waveguiding optics, both taken $170 \mathrm{~mm}$ behind the focal spot of the KB optics illustrating the filtering properties of the $2 D$ waveguide structure.

far-field profiles were investigated in more detail by orthogonal line scans using scintillation counters. The measured scans through the far-field both in vertical and horizontal direction are shown in Fig. 6.14 (symbols). The lines in the graph represent an integration of the simulated far-field in one direction (corresponding to the perpendicular scan direction). The simulated farfield intensity distribution was calculated from the simulated field distribution at the exit of the device as described in section 3.4. The simulations were scaled to the measured intensity. The scaling factor was determined for the vertical direction and then applied to the horizontal direction as well. Since the calculations were performed with a grid spacing of $1 \mathrm{~nm}$ both in vertical and horizontal direction, the 'field of view' during the calculation was reduced to $800 \times 800 \mathrm{~nm}^{2}$ in order to reduce computing time. Due to this, the background is too low in the simulations compared to the measurements. The measured background was therefore added to the simulation. The calculation is in very good agreement with the measured data concerning the vertical scan direction, while small deviations are observed between the simulation and the tails of the horizontal far-field distribution. The vertical side length of the rectangular WG was $30 \mathrm{~nm}$. This implies guiding of a single mode only. Therefore, small variations of the incidence angle $\theta_{i}$ (see Fig. 4.10) do not affect the shape of the far-field in this direction. In contrast, the horizontal dimension of the guide was $70 \mathrm{~nm}$, i.e. the guide exhibits multimodal behavior. Due to mode mixing in the 

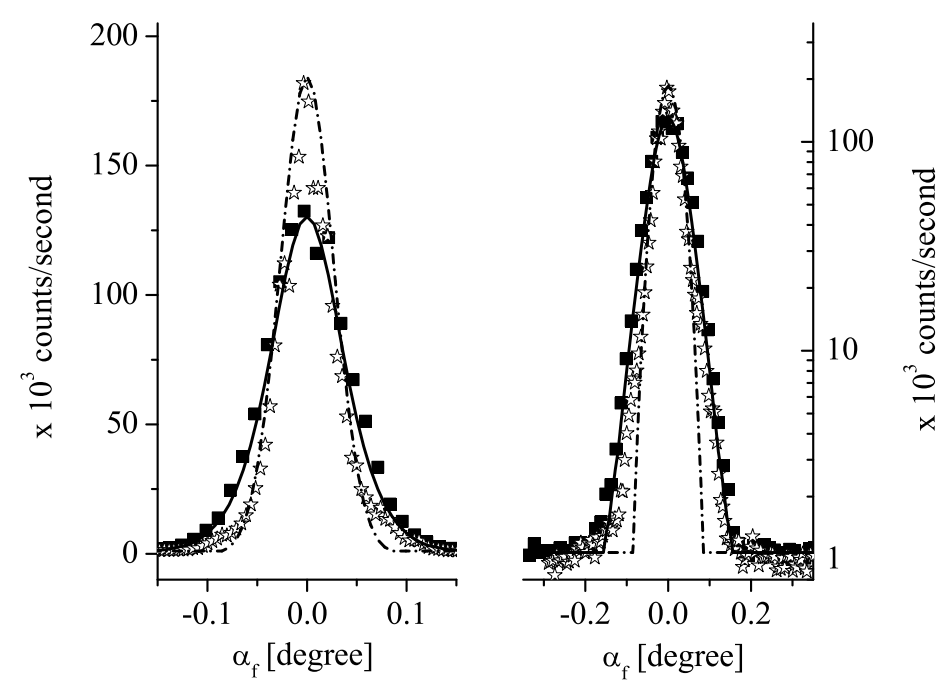

Figure 6.14: Measured far-field pattern of the waveguided beam in vertical (solid squares) and horizontal (open stars) direction, both in linear (left) and logarithmic (right) scales. The vertical axis denoted with $\alpha_{f}$ represents the farfield angles in vertical and horizontal direction respectively. The lines show the simulated far-field of a 2DXWG with a PMMA guiding layer of $30 \times 70 \mathrm{~nm}^{2}$ at an x-ray energy of $12.5 \mathrm{keV}$ (solid: vertical, dashed: horizontal). These values correspond to a beam size of $25 \times 47 \mathrm{~nm}^{2}$ (FWHM) in the near-field and directly behind the 2DXWG structure.

guide, the shape of the far-field is highly sensitive to the incidence angle $\varphi_{i}$, which explains the observation.

The beamsize at the exit of the waveguide (Full width at Half Maximum, FWHM) is $25 \times 47 \mathrm{~nm}^{2}$ which is the smallest beamsize for hard x-rays published so far. Owing to the filtering properties of the waveguide, this beam is not accompanied by disturbing side peaks or spurious reflected and primary beams, which would present a source of complication in imaging or diffraction applications. 


\section{Chapter 7}

\section{Summary}

X-ray waveguides consist of a guiding layer (one dimensionally confining waveguides, $1 \mathrm{DWG})$ or a guiding core $(2 \mathrm{DWG})$ which is incorporated into an adequate cladding material. The propagation of electromagnetic waves such as x-rays is restricted by boundary conditions. These lead to the formation of modes. It can be shown that the total number of modes is limited by the waveguides materials and dimensions. Within the scope of the present theses, hard x-ray $\left(\mathrm{E}_{\text {photon }}>5 \mathrm{keV}\right)$ waveguides have been studied in two different experimental setups:

In the resonant beam coupling scheme (RBC) the impinging x-ray beam is coupled via a thin top cladding layer into a one dimensionally confining waveguide using the evanescent wave in the cladding to couple into the guiding layer. This coupling scheme intrinsically provides a flux density (photons $/ \mathrm{s} / \mathrm{mm}^{2}$ ) enhancement of one to two orders of magnitude, depending on the experimental parameters such as the materials. The RBC concept can be generalized from monochromatic to white beam illumination of the waveguide. Using a white synchrotron radiation beam, all supported modes of the waveguide are excited at the same time but separated in their energetic position. Upon shining a white beam on $1 \mathrm{D}$ RBC waveguides one can benefit from exploiting the full energetic acceptance (EA) of the devices. The bandpass is approximately in the range of $\Delta \lambda / \lambda \sim 10^{-2}-10^{-3}$ again depending on the waveguide and on the mode number. The guided beam thus provides a convenient monochromatization corresponding to the EA. The energetic position of the excited modes can be tuned by changing the incidence angle $\alpha_{i}$ of the impinging white x-ray beam. The total flux per mode is further enhanced when compared to monochromatic illumination since the monochromacy of a synchrotron beam is typically achieved by perfect crystals providing $\Delta \lambda / \lambda \sim 10^{-4}$. A monomodal 
waveguide e.g. can thus be used as a quasi monochromatic divergent line source. The opening angle of the exiting beam is in the range of $\sim 1-3 \mathrm{mrad}$. Multimodal waveguides in turn could be used in future to perform experiments with enhanced energetic resolution compared to state of the art energy dispersive detectors (energy resolution typically $\sim 200 \mathrm{eV}$ ). At fixed detector position each mode represents one point in reciprocal space which e.g. allows for the observation of time dependent processes with enhanced energetic resolution. The experiments with RBC waveguides in white synchrotron radiation beams showed that the optical properties of these devices can be accurately simulated. The experimental observations and the simulated data were found to be in good agreement.

The RBC coupling scheme has previously been generalized to two dimensionally confining waveguides but in the proof-of-principle experiment the exiting beam of these devices was found to be of too small intensity for application as the intrinsic flux density enhancement could not compensate the loss of photons due to the small geometric acceptance of these devices. Furthermore, the exiting beam was accompanied by spurious transmitted and specular reflected beams which present a source of complication for imaging experiments.

To overcome these limitations, a new coupling scheme was tested changing from $\mathrm{RBC}$ to front coupling where the beam is coupled directly into the front side of the waveguide, using efficient prefocussing by curved mirror systems. Two dimensionally confining front coupling waveguides require high aspect ratios (length of the guiding core over its width) since the over illuminated radiation (those photons that do not hit the guiding core but the cladding) must be damped out by the cladding over the length of the waveguide. For hard x-rays aspect ratios of $\sim$ $10^{3}-10^{5}$ are needed to achieve the desired absorption. Different fabrication techniques have been tested to meet these requirements: templating from silk fibers using the fiber as guiding core, drilling of 2DWGs in metal foils using a focused ion beam and lithographic fabrication techniques. The latter turned out to provide the best fabrication results: Electron beam lithography was used to prepare 'airfilled' waveguides in Si by etching small grooves into a Si-wafer and subsequent covering of the grooves by wafer bonding. Furthermore polymer core waveguides were fabricated using small resist stripes left on a Si-substrate after electron beam lithography and further processing. Both devices were analyzed experimentally. The experimental data was compared to numerical simulations concerning the efficiency (flux in front of the guide over the flux at its exit) and the farfield distribution of the guided radiation. Both were found to be in good agreement with the simula- 
tions. With polymer core waveguides, the smallest hard x-ray beam published so far could be observed yielding a flux of $3.5 \cdot 10^{6}$ photons/s in a cross section of $25 \times 47 \mathrm{~nm}^{2}(\mathrm{FWHM})$ at the exit of the waveguide. In this setup the waveguide no longer provides an intrinsic flux density enhancement which therefore has to be provided by external focusing optics, namely - in case of the present work - a focusing Kirkpatrick-Baez mirror system. Combining these two techniques, a flux density enhancement of $g \sim 4000$ could be achieved at a waveguide efficiency of $\sim 5 \%$. In future, the total number of photons exiting the 2DWG could be further enhanced using a 'pink' synchrotron radiation beam, i.e. the radiation provided by an undulator without further monochromatization. For applications such as scanning fluorescence microscopy or in line holography the relaxed monochromaticity conditions would be tolerable while the enhanced flux would optimize the perfomance. We estimate that a total flux of $10^{8}$ photons per second would be possible in this case. Closing with a few numbers we can estimate the maximum throughput by a comparison with the brilliance B (photons $/$ second $/ \mathrm{mm}^{2} / \mathrm{s} / \mathrm{mrad}^{2} / 0.1 \%$ bandwidth) of a typical undulator. With $\mathrm{B}_{\text {unulator }} \simeq 10^{20}$ the maximum number of photons exiting a $10 \times 10 \mathrm{~nm}^{2}$ waveguide with an exit angle of $2 \mathrm{mrad}$ would then be $\simeq 2 \cdot 10^{10}$ at $\Delta \lambda / \lambda=10^{-2}$ (pink beam) and at an efficiency of $5 \%$. The brilliance of a Self Amplified Spontaneous Emission Free Electron Laser (SASE-FEL, $\mathrm{B}_{\text {peak, SASE-FEL }} \geq 10^{30}, \mathrm{~B}_{\text {average, SASE-FEL }} \geq 10^{24}$ ) could further increase this number by orders of magnitude. 


\section{Bibliography}

M. Abramowitz and I. A. Stegun. Handbook of Mathematical Functions. Dover, New York, 1965. ISBN 3-87144-818-4.

J. Als-Nielsen. Elements of Modern X-Ray Physics. Wiley, New York, 2001. ISBN 0-471-49857-2.

D. Attwood. Soft X-rays and extreme ultraviolet radiation. Cambridge University Press, 2000. ISBN 0-521-65214-6.

C. Bergemann, H. Keymeulen, and J. F. van der Veen. Focusing x-ray beams to nanometer dimensions. PRL, 91:204801, 2003.

D. Bilderback and D. Thiel. Microbeam generation with capillary optics. Rev. Sci. Instr., 66:2059, 1995.

M. Born and E. Wolf. Principles of Optics. Cambridge University Press, 1980. ISBN 0-521-642221.

Y. Dabin, G. Rostaing andO. Hignette, A. Rommeveaux, and A. Freund. The present state of kirkpatrick-baez mirrors at the ESRF. Proc. SPIE, 4782:235, 2002.

C. David, T. Weitkamp, B Nöhammer, and J. F. van der Veen. Diffractive and refractive x-ray optics for microanalysis applications. Spectrochimica Acta B, 59: $1505,2004$.

S. Di Fonzo, W. Jark, S. Lagomarsino, C. Giannini, L. Di Caro, A. Cedola, and M. Müller. Non-destructive determination of local strain with 100-nanometre spatial resolution. Nature, 403:638, 2000.

European Synchrotron Radiation Facility ESRF. http://www.esrf.fr. 
C. W. Extrand. Spin coating of very thin polymer films. Polym. Eng. Sci, 34:390, 1994.

E. Di Fabrizio, F. Romanetto, M. Gentili, S. Cabrini, B. Kaulich, J. Susini, and R. Barrett. High-efficiency multilevel zone plates for kev x-rays. Nature, 401: 895, 1999.

Y. P. Feng, S. K. Sinha, H. W. Deckman, J. B. Hastings, and D. P. Siddons. X-ray flux enhancmenz in thin-film waveguides using resonant beam couplers. Phys. Rev. Let., 71:537, 1993.

C. Fuhse, A. Jarre, C. Ollinger, J. Seeger, T. Salditt, and R. Tucoulou. Frontcoupling of a prefocused x-ray beam into a monomodal planar waveguide. Appl. Phys. Lett., 85:1907, 2004.

C. Fuhse and T. Salditt. Finite-difference field calculations for one-dimensionally confined x-ray waveguides. Physica B, 357:57, 2004.

A. Glisovic. Stuktur und Aufbau von Spinnenseide. Diplomarbeit, 2004.

O. Hignette, P. Cloetens, W.-K. Lee, and G. Rostaing. Hard x-ray microscopy with reflecting mirrors status and perspectives of the ESRF technology. J. Phys. IV, 104:231, 2003.

G. Ice, J. Chung, J. Tischler, A. Lunt, and L. Assouid. Elliptical x-ray microprobe mirrors by differential deposition. Rev. Sci. Instrum., 71:2635, 2000.

A. Jarre, C. Fuhse, C. Ollinger, J.Seegar, R. Tucoulou, and T. Salditt. Twodimensional hard x-ray beam compression by combined focusing and waveguide optics. Phys. Rev. Lett, 94:074801, 2005.

A. Jarre, T. Salditt, T. Panzner, U. Pietsch, and F. Pfeiffer. White beam x-ray waveguide optics. Appl. Phys. Lett, 85:161, 2003.

W. Kern. Handbook of semiconductor wafer cleaning. Noyes Publications, 1993. ISBN 0-8155-1331-3.

P. Kirkpatrick and A. V. Baez. Formation of optical images by x-rays. J. of the opt. soc of America., 38:766, 1948. 
Y. V. Kopylov, A. V. Popov, and A. V. Vinogradov. Application of the parabolic wave equation to x-ray diffraction optics. Opt. Comm., 118:619, 1995.

I. Langmuir. The vapor pressure of metallic tungsten. Phys. Rev. 2, 5:329, 1913.

I. Langmuir. The evaporation, condensation and reflection of molecules and the mechanism of adsorption. Phys. Rev. 8, 2:149, 1916.

B. Lengeler, C. G. Schroer, M. Kuhlmann, B. Benner, T. Günzler, O. Kurapova, F. Zontone, A. Snigirev, and I. Snigireva. Refractive x-ray lenses. J. Phys. D: Appl. Phys., 38:A218, 2005.

B. Lengeler, C. G. Schroer, M. Richwin, J. Tümmler, M. Drakopoulos, A. Snigirev, and I. Snigireva. A microscope for hard x rays based on parabolic compound refractive lenses. Appl. Phys. Lett., 74:3924, 1999.

Y. Li, M. Yasa, O. Pelletier, C. R. Safinya, E. Caine, E. E. Hu, and P. Fernandez. Metal layer bragg-fresnel lenses for diffraction focusing of hard x-rays. Appl. Phys. Lett., 82:2538, 2003.

A. Mara, Z. Siwy, C. Trautmann, J. Wan, and F. Kamme. An asymmetric polymer nanopore for single molecule detection. Nano Letters, 4:497, 2004.

D. Marcuse. Theory of dielectric optical waveguides. Academic Press, New York, 1974. ISBN 0-12-470950-8.

C. Ollinger, C. Fuhse, A. Jarre, and T. Salditt. Two dimensional x-ray waveguides on a grating. Physica B, 94:53, 2005.

T. Panzner, W. Leitenberger, J. Grenzer, Y. Bodenthin, T. Geue, U. Pietsch, and H. Möhwald. Coherence experiments at the energy-dispersive reflectometry beamline at bessy ii. J. Phys. D, 36:A93, 2003.

M. Peuker. High efficiency nickel phase zone plates with $20 \mathrm{~nm}$ minimum outermost zonewidth. Appl. Phys. Lett., 78:2209, 2001.

F. Pfeiffer. X-ray waveguides. Diplomarbeit, 1999.

F. Pfeiffer. X-ray and neutron waveguides. Dissertation, 2002.

F. Pfeiffer, C. David, M. Burghammer, and T. Salditt. Science, 297:230, 2002. 
U. Pietsch, J. Grenzer, T. Geue, F.Neissendorfer, G. Brezesinski, C. Symietz, H. Möhwald, and W. Gudat. The energy-dispersive reflectometer at bessy ii: a challenge for thin film analysis. Nucl. Instrum. Methods A, 47:777, 2001.

W. C. Röntgen. Über eine neue Art von Strahlen. Vorläufige Mitteilung. Sitzungsberichte der Würzburger Physikalischen-Medicinischen Gesellschaft, 137:132, 1895.

W. C. Röntgen. On a new kind of rays. Nature, 53:274, 1896.

T. Salditt, F. Pfeiffer, H. Perzl, A. Vix, U. Mennicke, A. Jarre, A. Marzuelas, and T.H. Metzger. X-ray waveguides and thin macromolecular films. Physica B, 336: 181, 2003.

C. G. Schroer, M. Kuhlmann, U. T. Hunger, T. F. Günzler, O. Kurapova, S. Feste, F. Frehse, B. Lengeler, M. Drakopoulos, A. Somogyi, A. S. Simionovici, A. Snigirev, and I. Snigireva. Nanofocusing parabolic refractive x-ray lenses. Appl. Phys. Lett., 82:1485, 2003.

C. G. Schroer, M. Kuhlmann, U. T. Hunger, T. F. Günzler, O. Kurapova, S. Feste, B. Lengeler, M. Drakopoulos, A. Somogyi, A. S. Simionovici, A. Snigirev, and I. Snigireva. Nanofocusing parabolic refractive x-ray lenses. Synchrotron Radiation Instrumentation, Conf. Proc., 705:740, 2004.

C. G. Schroer, O. Kurapova, J. Patommel, P. Boye, J. Feldkamp, B. Lengeler, M. Burghammer, C. Riekel, L. Vincze, A. van der Hart, and M. Küchler. Hard X-ray nanoprobe based on refractive x-ray lenses. Appl. Phys. Lett., 87:124103, 2005.

C. G. Schroer and B. Lengeler. Focusing hard x rays to nanometer dimensions by adiabatically focusing lenses. Phys. Rev. Lett., 94:054802, 2005.

J. Seeger. Nanolithographische Herstellung von 2D Wellenleitern für harte Röntgenstrahlen. Diplomarbeit, 2004.

A. Snigirev. The recent development of Bragg-Fresnel crystal optics. Experiments and applications at the ESRF. Rev. Sci. Instrum., 66:2053, 1994.

A. Snigirev, V. Kohn, I. Snigireva, and B. Lengeler. A compuond refractive lens for focusing high-energy x-rays. Nature, 384:49, 1996. 
E. Spiller and A. Segmüller. Propagation of x rays in waveguides. Appl. Phys. Lett., 24:60, 1974.

J. Susini. Design parameters for hard X-ray mirrors. Opt.Eng, 34:2361, 1995.

Q.-Y. Tong and U. Gösele. Semiconductor wafer bonding. Wiley \& Sons, New York, 1998. ISBN 0-471-57481-3.

K. Williams and R. Muller. Etch rates for micromachining processing. Journal of Microelectromechanical Systems, 5:256, 1996.

D. Windt. Imd-software for modeling the optical properties of multilayer films. Computers in Physics, 12:360, 1998.

D. Windt. imd 4.1.1, 2000, http://cletus.phys.columbia.edu/windt/idl. 2000.

M. Yamamoto, Y. Iriye, K. Asaumi, M. Shikida, Y. Matsushima, T. Yamashiro, and K. Sato. Characterization of orientation-dependent etching properties of single-crystal silicon: Effects of $\mathrm{KOH}$ concentration. Sensors and Actuators A, 64:87, 1988.

K. Yamauchi, K. Yamamura, H. Mimura, Y. Sano, A. Saito, K. Endo, A. Souvorov, M. Yabashi, K. Tamasaku, T. Ishikawa, and Y. Mori. Two-dimensional submicron focusing of hard x-rays by two elliptical mirrors fabricated by plasma chemical vaporization machining and elastic emission machining. Jpn. J. Appl. Phys., 42:7129, 2003.

K. Yamura, K. Yamauchi, H. Mimura, Y. Sano, A. Saito, K. Endo, A. Souvorov, M. Yabashi, K. Tamsako, T. Ishikawa, and Y. Mori. Fabrication of elliptical mirror at nanometer-level accuracy for hard x-ray focusing by numerically controlled plasma chemical vaporization machining. Rev. Sci. Instr., 74:4549, 2003.

M. Yasa, Y. Li, C. Mammen, J. Als-Nielsen, J. Hoszowska, C. Mocuta, and A. Freund. Double focusing of hard x rays using combined multilayer and bragg-fresnel optics. Appl. Phys. Lett, 84:4744, 2004.

W. Yun, B. Lai, J. Maser, D. Legnini, E. Gluskin, Z. Chen, A. Krasnoperova, Y. Vladimirsky, F. Cerrina, E. Di Fabrizio, and M. Gentili. Nanometer focusing of hard x rays by phase zone plates. Rev. Sci. Instr., 70:2238, 1999. 
M. J. Zwanenburg, J. F. Peters, J. H. H. Bongaerts, S. A. de Vries, D. A. Abernathy, and J. F. van der Veen. Coherent propagation of x-rays in a planar waveguide with a tunable air gap. Phys. Rev. Lett., 82:1696, 1999. 


\section{Acknowledgements}

In concluding this thesis, I want to acknowledge the help of my supervisor, my family and friends, and my colleagues who supported me during the course of my $\mathrm{PhD}$ program.

First of all, I thank Prof. Tim Salditt for proposing such an interesting and rewarding research topic. I enjoyed the nice atmosphere, many fruitful discussions and exciting beamtimes together with him.

It was a pleasure to work with the members of the 'waveguide team' Christian Fuhse, Christoph Ollinger and Jens Seeger whom I want to thank for excellent team work. Their assistance in proofreading the thesis was a great help. Further thanks to Anja Glisovic for providing spiders and silk.

For technical and logistic assistance I want to thank Jochen Herbst, Thorsten Gronemann, Peter Nieschalk, Jens Wegener, Hans Düben, Judith Rech, Michaela Ständer, Sabine Balder and Christina Emser-Spuddik.

I greatfully acknowledge the help of Dr. Christian David who contributed with his excellent knowledge on e-beam lithography and nanostructuring.

Many thanks are also due to Prof. Ulrich Pietsch, Tobias Panzner and Yves Bodenthin from Potsdam University for their support during numerous beamtimes at their edr beamline at BESSY II.

At last, I am very thankful to my parents whose love and support has been with me throughout my life. 


\section{List of Publications}

- T. Salditt, F. Pfeiffer, H. Perzl, A. Vix, U. Mennicke, A. Jarre, A. Marzuelas and T.H. Metzger $X$-ray waveguides and thin macromolecular films, Physica B. 336, 181 (2003).

- A. Jarre, T. Salditt, T. Panzner, U. Pietsch and F. Pfeiffer White beam x-ray waveguide optics, Appl. Phys. Lett. 85, 161 (2004).

- C. Fuhse, A. Jarre, C. Ollinger, J. Seeger, T. Salditt and R. Tucoulou

Front-coupling of a prefocused $x$-ray beam into a monomodal planar waveguide,

Appl. Phys. Lett. 85, 1907 (2004).

- C. Ollinger, C. Fuhse, A. Jarre and T. Salditt

Two dimensional $X$-ray waveguides on a grating,

Physica B. 94, 53 (2005).

- A. Jarre, C. Fuhse, C. Ollinger, J.Seeger, R. Tucoulou and T. SALDITT

Two-dimensional hard X-ray beam compression by combined focusing and waveguide optics,

Phys. Rev. Lett. 94, 074801 (2005). 



\section{Curriculum Vitae}

Name: Ansgar Clemens Jarre

Born in Rheinfelden (Baden), Germany, on August 21 ${ }^{\text {st }}, 1972$.

09.1979 - 08.1983 Karl Tschamber Elementary School, Weil am Rhein

09.1983 - 06.1992 Kant Gymnasium, Weil am Rhein

07.1990 - 10.1990 Ashville College, Harrogate, England

07.1992 - 10.1992 Basic training (Air base Mengen)

11.1992 - 10.1993 Civil Service (Old people's home, Weil am Rhein)

10.1993 - 09.2001 Ludwig-Maximilians-Universität Munich (Germany)

(Diplom in Physics)

10.2001 - 11.2002 University of Saarbrücken

11.2002 - 06.2005 Georg August University, Göttingen 